\title{
Microcanonical Simulations of Adsorbing Self-Avoiding Walks
}

\author{
E.J. Janse van Rensburg $1 \dagger$ \\ ${ }^{1}$ Department of Mathematics and Statistics, York University, Toronto, Ontario \\ M3J 1P3, Canada
}

\begin{abstract}
Linear polymers adsorbing on a wall can be modelled by half-space selfavoiding walks adsorbing on a line in the square lattice, or on a surface in the cubic lattice. In this paper a numerical approach based on the GAS algorithm is used to approximately enumerate states in the partition function of this model. The data are used to approximate the free energy in the model, from which estimates of the location of the critical point and crossover exponents are made. The critical point is found to be located at

$$
a_{c}^{+}= \begin{cases}1.779 \pm 0.003, & \text { in the square lattice } \\ 1.306 \pm 0.007, & \text { in the cubic lattice }\end{cases}
$$

These results are then used to estimate the crossover exponent $\phi$ associated with the adsorption transition, giving

$$
\phi= \begin{cases}0.496 \pm 0.009, & \text { in two dimensions; } \\ 0.505 \pm 0.006, & \text { in three dimensions. }\end{cases}
$$

In addition, the scaling of these thermodynamic quantities is examined using the numerical data, including the scaling of metric quantities, and the partition and generating functions. In all cases results and numerical values of exponents were obtained which are consistent with estimates in the literature.

PACS numbers: 82.35.Lr,82.35.Gh,61.25.Hq
\end{abstract}

AMS classification scheme numbers: 82B41, 82B80, 65C05

Submitted to: J. Stat. Mech. Theor. Exp. 


\section{Introduction}

The adsorption of a linear polymer on an attractive surface is a conformational rearrangement of the polymer to a state where it explores conformations which remain near or on the surface. This is the so-called polymer adsorption transition, and the many models of this phenomenon (see, for example, reference [22]) remain a rich source of mathematical and numerical studies. Polymer adsorption is a phase transition [8], and the properties of the adsorbed polymer have been examined both experimentally (see, for example, references [7, 13, 32]) and theoretically [12]. These models include directed path models of adsorbing polymers [35, 40], as well as self-avoiding walk models [18], and these have received considerable attention in the literature [19], using both rigorous methods [38] and numerical methods (for example, the Monte Carlo simulation of adsorbing self-avoiding walks [23]).

In this study, a new Monte Carlo method for sampling adsorbing self-avoiding walks is proposed and implemented. In particular, the GAS algorithm [24] is generalised to sample adsorbing walks in the microcanonical ensemble, and the data obtained are analysed to estimate the locations of critical points, and the values of critical exponents and scaling of adsorbing walks, in the square and cubic lattices. The algorithm is related to the Rosenbluth algorithm [37] and to the GARM algoritm [36]. The GARM algorithm is related to the PERM algorithm [15,20], and flat histogram implementations of PERM [33] have been used to sample states from a flat histogram over state space in a variety of different models of interacting walks, including collapsing walks [34] and adsorbing walks [28].

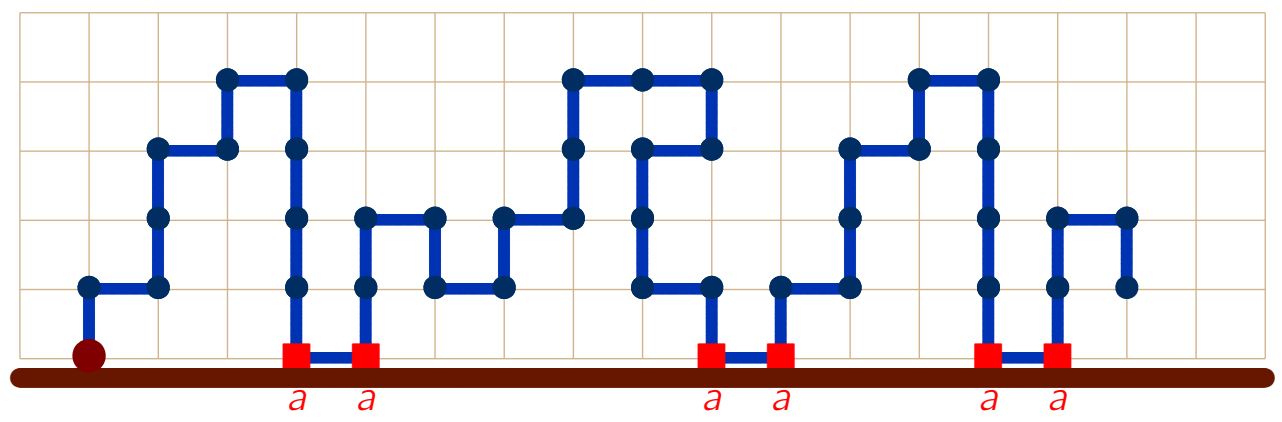

Figure 1: An adsorbing self-avoiding walk in the half square lattice $\mathbb{L}_{+}^{2}$. The walk steps from the origin in the lattice, has length 46 , and makes 6 visits to the adsorbing line $\partial \mathbb{L}_{+}^{2}$ (the boundary of $\mathbb{L}_{+}^{2}$ ). The weight of this walk in the partition function $Z_{46}(a)$ is $a^{6}$.

The GAS algorithm was introduced in reference [24] and was used in several studies as an algorithm to approximately enumerate walks or polygons 25, 26]. However, it was not clear how to generalise the algorithm to sample states in models of interacting walks. In this paper our purpose is (1) to generalise the algorithm to a model of adsorbing walks, (2) to examine the behaviour of the algorithm by computing critical points and exponents of the walk, and to compare this to results found elsewhere, and (3) to use our data to examine scaling in adsorbing walks by computing critical exponents and examining the scaling of thermodynamic functions. 


\subsection{Adsorbing walks}

An adsorbing self-avoiding walk in the square lattice is illustrated in figure 1 . Let $\mathbb{L}^{d}$ denote the $d$-dimensional hypercubic lattice and denote a unit length edge with endpoints $\vec{x}$ and $\vec{y}$ in $\mathbb{L}^{d}$ by $\langle\vec{x} \sim \vec{y}\rangle$. The hypercubic half-lattice $\mathbb{L}_{+}^{d}$ is defined by

$$
\mathbb{L}_{+}^{d}=\left\{\langle\vec{x} \sim \vec{y}\rangle \in \mathbb{L}^{d} \mid \vec{x}(d) \geq 0 \text { and } \vec{y}(d) \geq 0\right\},
$$

where $\vec{x}(d)$ is the $d$-th Cartesian component of $\vec{x}$, and $\vec{y}(d)$ is the $d$-th Cartesian component of $\vec{y}$. The boundary of $\mathbb{L}_{+}^{d}$ is given by

$$
\partial \mathbb{L}_{+}^{d}=\left\{\langle\vec{x} \sim \vec{y}\rangle \in \mathbb{L}_{+}^{d} \mid \vec{x}(d)=0 \text { and } \vec{y}(d)=0\right\},
$$

and it is isomorphic to $\mathbb{L}^{d-1}$ if $d \geq 2$. Please note that these definitions, and the definitions of additional functions and quantities, are listed in table 1.

The number of self-avoiding walks of length $n$ from the origin in $\mathbb{L}^{d}$ is denoted by $c_{n}$. The growth constant of the self-avoiding walk [16, 17] is defined by the limit

$$
\lim _{n \rightarrow \infty} c_{n}^{1 / n}=\mu_{d}
$$

and $\kappa_{d}=\log \mu_{d}$ is the connective constant of the self-avoiding walk. This shows that $c_{n}=\mu_{d}^{n+o(n)}$. Self-avoiding walks from the origin in $\mathbb{L}_{+}^{d}$ are positive walks. The number of positive walks of length $n$ is denoted by $c_{n}^{+}$, and it is known that $c_{n}^{+}=\mu_{d}^{n+o(n)}[18]$.

If positive walks are counted with respect to the number of vertices in $\partial \mathbb{L}_{+}^{d}$ (these are visits), then the walks are adsorbing walks. For example, the walk in figure 1 is an adsorbing walk with 6 visits.

Let $c_{n}^{+}(v)$ be the number of adsorbing walks of length $n$ from the origin in $\mathbb{L}_{+}^{d}$, with $v$ visits to $\partial \mathbb{L}_{+}^{d}$. The canonical partition function of adsorbing walks is obtained by introducing an activity a conjugate to the number of visits:

$$
Z_{n}(a)=\sum_{v=0}^{n} c_{n}^{+}(v) a^{v} .
$$

When $a$ is large, then $Z_{n}(a)$ is dominated by walks with a large number of visits, and if a is small (but positive), then $Z_{n}(a)$ is dominated by walks with a small number of visits.

The finite size free energy of these models is computed from the partition function $Z_{n}($ a) (see equation (6)), and is given by

$$
\mathcal{F}_{n}(a)=\frac{1}{n} \log \sum_{v} c_{n}^{+}(v) a^{v} .
$$

The limiting free energy of the model is given by the thermodynamic limit in the model:

$$
\mathcal{F}(a)=\lim _{n \rightarrow \infty} \frac{1}{n} \log \sum_{v} c_{n}^{+}(v) a^{v} .
$$

This limit exists (see reference [18], and also, for example, reference [22]), and it is a convex function of log a with a singular point at $a=a_{c}^{+}$(which is the adsorption critical point in the model). For $a<a_{c}^{+}$the model is in a desorbed state, and for $a>a_{c}^{+}$the model is in an adsorbed state. It is known that $a_{c}^{+}>1[21]$, and $a_{c}^{+}<\frac{\mu_{d}}{\mu_{d-1}}[18]$, and

$$
\mathcal{F}(a) \begin{cases}=\log \mu_{d}, & \text { if } a \leq a_{c}^{+} ; \\ >\log \mu_{d}, & \text { if } a>a_{c}^{+} .\end{cases}
$$


Since $\mathcal{F}(a)$ is a convex function of $\log a$, it is differentiable for almost all $a>0$, and it follows that the density of visits to the adsorbing plane is $a \frac{d}{d a} \mathcal{F}(a)=0$ if $a<a_{c}^{+}$(this is the desorbed phase), and $a \frac{d}{d a} \mathcal{F}(a)>0$ if $a>a_{c}^{+}$almost surely (whenever $\mathcal{F}(a)$ is differentiable). This is the adsorbed phase. In the desorbed phase the walk tends to make few returns to the adsorbing plane (walks of length $n$ will return, on average, $o(n)$ times to the adsorbing plane in the desorbed phase). Thus, a desorbed walk will tend to move away from the boundary into the bulk of $\mathbb{L}_{+}^{d}$. An adsorbed walk, on the other hand, is expected to have a positive density of returns to the adsorbing plane. This implies that the walk will remain near the adsorbing plane, and so have the properties of a walk which is stretched out in $(d-1)$ dimensions near $\partial \mathbb{L}_{+}^{d}$ (and compressed in the $d$-th dimension). Separating these two regimes is the adsorption critical point $a_{c}^{+}$.

The finite size scaling of the free energy $\mathcal{F}_{n}(a)$ is given by

$$
\mathcal{F}_{n}(a) \simeq \log \mu_{d}+\left(a-a_{c}^{+}\right)^{2-\alpha} f\left(n^{\phi}\left(a-a_{c}^{+}\right)\right)
$$

where $f$ is a scaling function, $\alpha$ is the specific heat exponent, and $\phi$ is the finite size crossover exponent. The exponents $\alpha$ and $\phi$ are related by the hyperscaling relation

$$
2-\alpha=\frac{1}{\phi}
$$

The bulk entropy contribution to $\mathcal{F}_{n}(a)$ is $\log \mu_{d}$ in $\mathbb{L}_{+}^{d}$ (where $\mu_{d}$ is the growth constant of self-avoiding walks in the square lattice). Slightly redefining the scaling function, it is found that

$$
\mathcal{F}_{n}(a) \simeq \log \mu_{d}+\frac{1}{n} g\left(n^{\phi}\left(a-a_{c}^{+}\right)\right) .
$$

By plotting $n\left(\mathcal{F}_{n}(a)-\log \mu_{d}\right)$ against $n^{\phi}\left(a-a_{c}^{+}\right)$, the function $g$ can be uncovered (for $n$ large and $\left|a-a_{c}^{+}\right|$small).

Taking derivatives of $\mathcal{F}_{n}(a)$ to log a gives the energy (density) $\mathcal{E}_{n}(a)$ and specific heat $\mathcal{C}_{n}(a)$ of the model. The scaling of these quantities follows directly from equation (12):

$$
\mathcal{E}_{n}(a) \simeq n^{\phi-1} h_{e}\left(n^{\phi}\left(a-a_{c}^{+}\right)\right), \quad \text { and } \quad \mathcal{C}_{n}(a) \simeq n^{\alpha \phi} h_{c}\left(n^{\phi}\left(a-a_{c}^{+}\right)\right),
$$

for some scaling functions $h_{e}$ and $h_{c}$. In the limit as $n \rightarrow \infty, \mathcal{E}_{n}(a) \rightarrow \mathcal{E}(a)$ (the limiting energy density) and $\mathcal{C}_{n}(a) \rightarrow \mathcal{C}(a)$ (the limiting specific heat). Existence of these limits (almost everywhere) is a consequence of the convexity properties of the limiting free energy (see for example reference [22]). Physically, $\mathcal{E}(a)$ is the density of visits per unit length, and $\mathcal{C}(a)$ is the rate of change in $\mathcal{E}(a)$ as a function of changes in log a (it has a maximum at $a_{c}^{+}$).

For adsorbing walks it is thought that $\phi=\frac{1}{2}$ in all dimensions $d \geq 2$ [2, 9], and numerical evidence supporting this in dimensions lower than $d=4$ (the upper critical dimension) are available in references $[4,23,27,29,31]$. If $\phi=\frac{1}{2}$, then $\alpha=0$, so, for example, the specific heat has scaling $\mathcal{C}_{n}(a)=h_{c}\left(n^{\phi}\left(a-a_{c}^{+}\right)\right)$, and plotting measurements of $\mathcal{C}_{n}(a)$ against the rescaled variable $\tau=n^{\phi}\left(a-a_{c}^{+}\right)$for small values of $\tau$ should collapse the curves to a limiting curve (with some finite size corrections to scaling), exposing the scaling function $h_{c}$. 
The partition function has a more complicated scaling law (see, for example, equation (23) in reference [23], or section 4.2 .2 in reference [22]). In the high temperature (or small a) regime, the partition function scales as

$$
Z_{n}(a) \simeq B_{\lambda} n^{\gamma_{t}-1} h_{\lambda}\left(n^{\phi}|t|\right) \kappa_{-}^{n|t|^{1 / \phi}}, \quad \text { if } a<a_{c}^{+},
$$

where $t=\left(a-a_{c}^{+}\right)$and $\lambda$ denotes the high temperature regime, and where $h_{\lambda}(x) \simeq$ $|x|^{\left(\gamma_{1}-\gamma_{t}\right) / \phi}$ and $\log \kappa_{-} \simeq\left|a-a_{c}^{+}\right|^{-1 / \phi} \log \mu_{d}$. Putting $a=1$, for example, and adsorbing constants and functions of $t$ into $B_{\lambda}$, give

$$
Z_{n}(1) \simeq B_{\lambda} n^{\gamma_{1}-1} \mu_{d}^{n}, \quad \text { since } \mathcal{F}(a)=\log \mu_{d} \text { if } a<a_{c}^{+},
$$

and the exponent $\gamma_{1}$ is the entropic exponent of half-space walks, namely $c_{n}^{+} \sim n^{\gamma_{1}-1} \mu_{d}^{n}$.

At the critical adsorption point $a_{c}^{+}$, the above scaling is modified to

$$
Z_{n}\left(a_{c}^{+}\right) \simeq B_{c} n^{\gamma_{t}-1} \mu_{d}^{n}, \quad \text { if } a=a_{c}^{+},
$$

where $\gamma_{t}$ is the entropic exponent associated with adsorbing walks at the critical adsorption point. The ensemble of half-space walks at the critical point has associated surface entropic exponent $\gamma_{s}$, and this is related to $\gamma_{t}$ by $\gamma_{t}=\gamma_{s}$ (see for example section 9.1.3 in reference [22]).

The scaling in the adsorbed phase is similar to the above, but now with different exponents

$$
Z_{n}(a) \simeq B_{\tau_{0}} n^{\gamma_{t}-1} h_{\tau_{0}}\left(n^{\phi}|t|\right) \kappa_{+}^{n|t|^{1 / \phi}}, \quad \text { if } a>a_{c}^{+},
$$

where $h_{\tau_{0}}(x) \simeq|x|^{\left(\gamma_{+}-\gamma_{t}\right) / \phi}$, and where the subscript $\tau_{0}$ denotes the low temperature (and large a) scaling. Since $\frac{1}{n} \log Z_{n}(a)=\mathcal{F}(a)(1+o(1))$, it follows that $\log \kappa_{+} \simeq$ $\left|a-a_{c}^{+}\right|^{-1 / \phi} \mathcal{F}(a)$. This, in particular, gives the scaling

$$
\mathcal{F}_{s}(a) \sim\left|a-a_{c}^{+}\right|^{1 / \phi}
$$

for the singular part of the free energy in the adsorbed phase (consistent with the hyperscaling relation (11)). The scaling of $Z_{n}(a)$ simplifies here to

$$
Z_{n}(a) \simeq B_{\tau_{0}} n^{\gamma_{+}-1} e^{n \mathcal{F}(a)}, \quad \text { if } a>a_{c}^{+} .
$$

The exponent $\gamma_{+}$should be that of adsorbed walks, and so given by $\gamma_{+}=\gamma^{(d-1)}$, the entropic exponent of walks in one dimension lower.

\subsection{Organisation of the manuscript}

This paper is a report on two aspects of the Monte Carlo simulation of adsorbing walks. The first is the generalisation of the GAS algorithm to a model of interacting walks, and in particular, an implementation of this algorithm to achieve flat histogram sampling over state space of adsorbing square and cubic lattice walks. The second aspect of the paper is a report on the properties of adsorbing self-avoiding walks in the square and cubic lattices. The aim here is to verify the results obtained in the Multiple Markov Chain Monte Carlo study in reference [23], and also to use the data generated here to test the scaling of the thermodynamic and metric quantities of adsorbing walks.

The model of adsorbing walks is defined in section 1.1. and its partition function and free energy are discussed. The limiting free energy of this model exists, and its properties 
Table 1: Short list of definitions

\begin{tabular}{l|l}
\hline Function & Definition \\
\hline $\mathbb{L}^{d}$ & The hypercubic lattice \\
$\mathbb{L}_{+}^{d}$ & The half-hypercubic lattice \\
$\partial \mathbb{L}_{+}^{d}$ & The boundary of $\mathbb{L}_{+}^{d}$ (it is isomorphic is $\left.\mathbb{L}^{d-1}\right)$ \\
$\mu_{d}$ & The growth constant of self-avoiding walks in $\mathbb{L}^{d}$ \\
$\mathcal{C}_{n}^{+}(v)$ & The number of positive walks of length $n$ and $v$ visits from $\overrightarrow{0}$ in $\mathbb{L}_{+}^{d}$ \\
$Z_{n}(a)$ & Partition function of positive adsorbing walks of length $n$ and activity a \\
$\mathcal{F}_{n}(a)$ & Finite size free energy: $F_{n}(a)=\frac{1}{n} \log Z_{n}(a)$ \\
$\mathcal{F}(a)$ & Limiting free energy: $\mathcal{F}(a)=\lim _{n \rightarrow \infty} \mathcal{F}_{n}(a)$ \\
$\mathcal{E}_{n}(a), \mathcal{E}(a)$ & Energy density and limiting energy density \\
$\mathcal{C}_{n}(a), \mathcal{C}(a)$ & Specific heat and limiting specific heat \\
$H_{n}$ & The mean height of the endpoint of the walk (a function of a) \\
$R_{n}^{2}$ & The mean square radius of gyration of the walk (a function of a) \\
$P^{+}(\epsilon)$ & The microcanonical density function (see equation 59$)$ \\
$a_{c}^{+}$ & The adsorption critical point \\
$\phi$ & The crossover exponent \\
$\alpha$ & The specific heat exponent \\
$\gamma_{1}$ & The half-space entropic exponent, see also $\gamma_{s}$ (the surface exponent) \\
$\nu$ & The metric exponent \\
$G(a, t)$ & Generating function of $Z_{n}(a)$ (see equation 66$)$ \\
$G_{N}(a, t)$ & Truncated generating function (see equation (67) $)$ \\
$t_{c}^{+}(a)$ & The radius of convergence of $G(a, t)$ \\
\hline
\end{tabular}

have been examined elsewhere [18]; see, for example, reference [22]. The basic scaling relations for the free energy, energy, and specific heat were introduced above, and the scaling of the partition function was briefly reviewed.

In section 2 the GAS algorithm [24] is reviewed and then generalised to interacting models. The algorithm normally has only one set of parameters (associated with the size of the walks), but it is shown here that introducing a second set of parameters (associated with the energy of the walks) can give an algorithm which samples effectively in both length and energy. It is shown that the algorithm can be tuned to give flat histogram sampling in the spirit of the PERM algorithm [15,20] (but without using enrichment or pruning of states).

Numerical results for adsorbing walks are analysed in section 3. The location of the adsorption critical point is determined from the mean energy of adsorbing walks, giving

$$
a_{c}^{+}= \begin{cases}1.779 \pm 0.003, & \text { in the square lattice; } \\ 1.306 \pm 0.007, & \text { in the cubic lattice }\end{cases}
$$




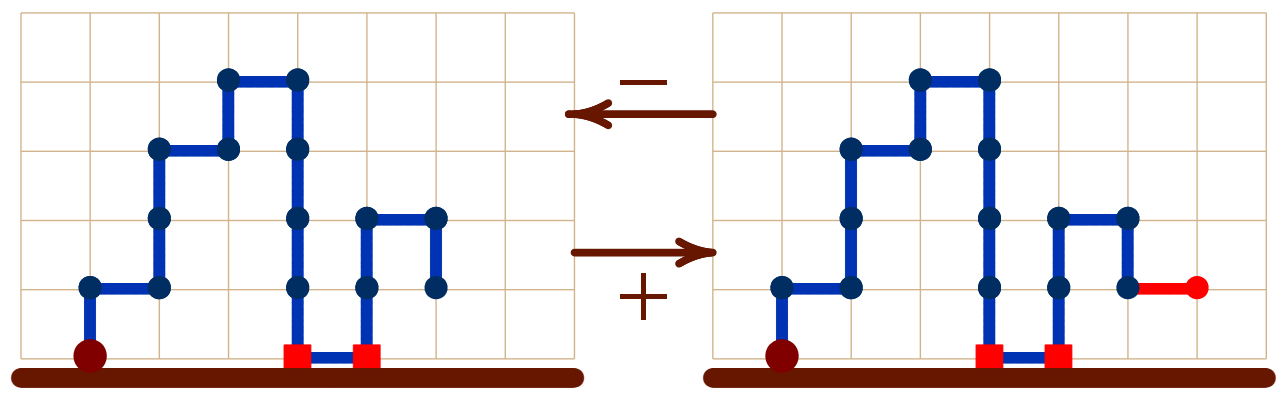

Figure 2: Endpoint elementary moves. Appending an edge at the endpoint of the walk on the left is a positive elementary move. Removing the last edge on the right is the reverse of a positive elementary move. This is a negative elementary move.

These results are then used to estimate the crossover exponent $\phi$ associated with the adsorption transition, giving

$$
\phi= \begin{cases}0.496 \pm 0.009, & \text { in two dimensions; } \\ 0.505 \pm 0.006, & \text { in three dimensions. }\end{cases}
$$

The microcanonical density function of adsorbing walks is determined as well, and shown to have properties consistent with the location of the critical points above. In addition, the specific heat of the model is determined, and it is found that it has scaling behaviour consistent with the estimates of the critical points above.

It is also shown that the adsorption transition is seen in a change in the metric scaling of walks at the critical point. The desorbed phase is a phase of positive walks with the scaling properties of self-avoiding walks in a good solvent, while the adsorbed walk has the metric properties of a walk in one dimension less; that is, of walks adhering to the adsorbing surface.

Scaling of the generating and partition functions are found to be consistent with the exact values of critical exponents determined elsewhere in two dimensions [2, 9], and with the value $\phi=\frac{1}{2}$ in three dimensions [19, 23, 31]. Similary, the data are also consistent with estimates for the surface exponent $\gamma_{s}$; this is seen particularly in the scaling of the partition function (see equation (16)).

The paper is concluded with a few brief remarks and a summary in section 4.

\section{GAS Sampling of self-avoiding walks}

The GAS algorithm is a generalisation of kinetic growth algorithms. It is designed to sample along weighted sequences in state space in such a way that the ratios of average weights of sequences ending in walks of length $n$ and $m$ are estimates of the ratio of the numbers of walks of lengths $n$ and $m$. In this section I show how to generalise this algorithm so that it can be used to estimate the number of walks of length $n$ and energy $m$. That is, the algorithm will be used to sample walks in the microcanonical ensemble.

Let $w=\left\langle\omega_{0}, \omega_{1}, \ldots, \omega_{n}\right\rangle$ be a self-avoiding walk of length $n$ from its source vertex $\omega_{0}=\overrightarrow{0}$ at the origin, to its terminal vertex $\omega_{n}$, giving $n$ steps $\left\langle\omega_{j-1}, \omega_{j}\right\rangle$ for $j=1,2, \ldots, n$. 


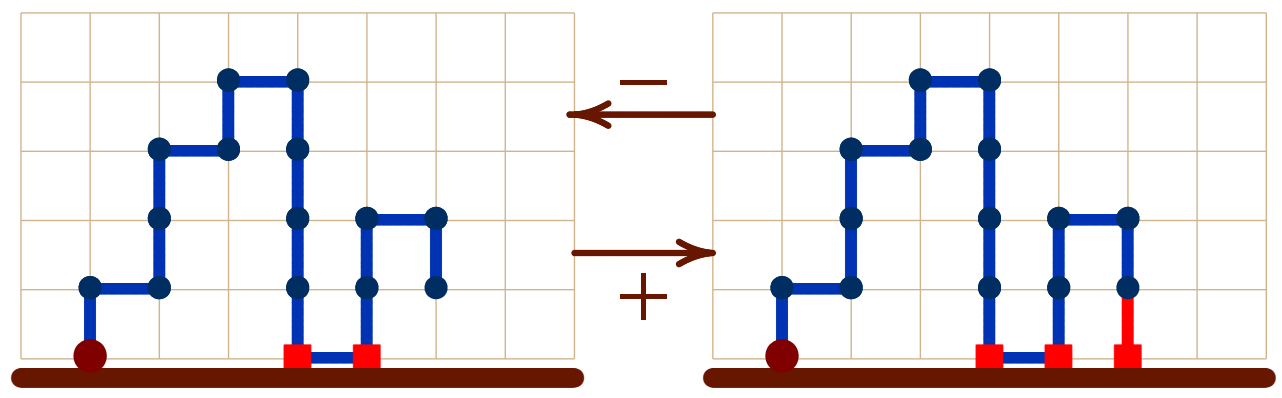

Figure 3: A endpoint elementary move may increase or decrease the number of visits in a walk. By appending an edge at the endpoint of the walk on the left a new visit is created. Removing the last edge on the right is the reverse of this move, and it decreases the number of visits by one.

The walk $w$ may be made longer by adding a step $\left\langle\omega_{n}, \omega_{n+1}\right\rangle$ to $\omega_{n}$, or it may be made shorter by removing its last step. These two operations compose an end-point elementary move for sampling self-avoiding walks, as illustrated in figure 2. A positive elementary move is the addition of an edge to the endpoint of a growing walk. The reverse of a positive elementary move is a negative elementary move, namely the deletion of the least edge in a walk. Notice that every positive move is immediately reversible by a negative elementary move.

Endpoint elementary moves have been used widely in the simulation of self-avoiding walks (for example the Rosenbluth algorithm [37], and the Beretti-Sokal algorithm [6]). In what follows the discussion will be restricted to endpoint elementary moves; however, the algorithms generalise directly if other elementary moves, such as BFACF elementary moves [1, 5], or generalised atmospheric moves [36], are used instead.

As an example, consider the elementary move in figure 2, which is an implementation of an endpoint elementary move on a self-avoiding walk in the positive half-lattice $\mathbb{L}_{2}^{+}$ (where $\vec{w}(2)$ is the $y$-coordinate of $\vec{w}$ ). The set of lattice edges in $\mathbb{L}_{2}^{+}$which may be appended to the walk $w$ to extend it by one step is the positive atmosphere of $w$, and the number of edges in the positive atmosphere is denoted by $a^{+}(w)$. For example, for the walk on the left in figure 2, $a^{+}(w)=2$.

Similarly, the set of edges which may be removed from the endpoint of a walk to decrease its length by one, is the negative atmosphere of the walk. For endpoint elementary moves, the last step is always the sole negative atmospheric edge, so that the size of the negative atmosphere for endpoint elementary moves is always $a^{-}(w)=1$, if $w$ is not the trivial walk of length 0 .

An elementary move may change the energy of a walk. For example, in a model of adsorbing walks in $\mathbb{L}_{2}^{+}$, the energy is the number of visits of the walk to the adsorbing line $\partial \mathbb{L}_{2}^{+}$(the boundary of the half-lattice $\mathbb{L}_{2}^{+}$). The positive move in figure 2 does not change the number of visits, but the move in figure 3 increases the number of visits (and so the energy) by 1 . The negative move in figure 3 similarly reduces the number of visits by 1 .

A similar situation arises if a model of collapsing walks with energy given by nearest neighbour contacts between vertices in the walk which are adjacent in $\mathbb{L}$, but not in the 


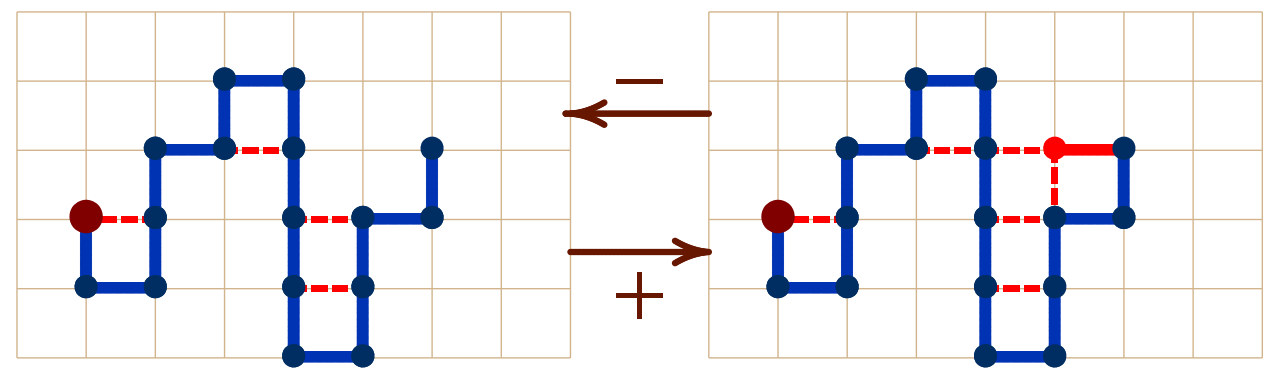

Figure 4: A endpoint elementary move may increase or decrease the number of contacts in a walk. By appending an edge at the endpoint of the walk on the left two new contacts are created. Removing the last edge on the right is the reverse of this move, and it decreases the number of contacts by two.

walk. This is illustrated in figure 4, the positive elementary move creates 2 new contacts in the walk, and so it changes the energy of the walk by 2 .

More general elementary moves (for example, the BFACF elementary moves) may contribute to the atmospheric statistics $a^{+}$and $a^{-}$in various ways, and may even give rise to neutral atmospheres which do not change the length of the walk (but which may change the energy of the walk).

Thus, in what follows, let $a_{v}^{+}(w)$ be the size of the positive atmosphere of a walk $w$ of length $\ell_{n}=|w|$, and which changes the energy of $w$ by $v$ units. For example, the walk on the left in figure 2 has $a_{0}^{+}(w)=1$ and $a_{1}^{+}(w)=1$, and if this was a walk in the three dimensional half-lattice $\mathbb{L}_{3}^{+}$, then $a_{0}^{+}(w)=3$ and $a_{1}^{+}(w)=1$. The walk on the left in figure 4 (with energy given by the number of contacts), has, in a similar way, $a_{0}^{+}(w)=2, a_{1}^{+}(w)=0$ and $a_{2}^{+}(w)=1$.

In exactly the same way one may define the size of the negative atmosphere of a walk $w$ of length $\ell$ which changes the energy by $v$, denoted by $a_{v}^{-}(w)$.

The neutral atmosphere (an elementary move which does not change the length of the walk) of a walk $w$ is similarly given by $a_{v}^{0}(w)$, if it changes the energy of a walk by $v$. If the endpoint elementary move in figure 2 is used, then $a_{v}^{0}(w)=0$ by default (since there are no neutral elementary moves implemented), but in general assume that a more general set of elementary moves (for example BFACF elementary moves) is used to sample walks, and in that case the neutral atmosphere may have positive size.

\subsection{Implementation of GAS-sampling}

Suppose that an elementary move is implementated on the state space of self-avoiding walks from the origin, and assume the implementation is irreducible (that is, the elementary move gives a connected graph on the state space of walks).

Suppose that the sequence

$$
\phi_{N}=\left\langle w_{0}, w_{1}, w_{2}, \ldots, w_{n}, \ldots, w_{N}\right\rangle
$$

is realised after $N$ steps and that the atmospheres of the states $w_{n}$ have sizes (or statistics) $a_{v}^{+}\left(w_{n}\right), a_{v}^{0}\left(w_{n}\right)$ and $a_{v}^{-}\left(w_{n}\right)$. These elementary moves may be classified as follows, depending on whether they increase or decrease the lengths of the walks (or are 
neutral), or whether they increase or decrease, or leave unchanged, the energy of the walk. This is done by defining atmospheric statistics as follows: Let the states $w_{n}$ be walks of length $\ell_{n}=\left|w_{n}\right|$. Define

$$
\begin{array}{rlrl}
\alpha_{n}^{--} & =\sum_{v<0} a_{v}^{-}\left(w_{n}\right), & \alpha_{n}^{-0}=\sum_{v=0} a_{v}^{-}\left(w_{n}\right), & \alpha_{n}^{-+}=\sum_{v>0} a_{v}^{-}\left(w_{n}\right) ; \\
\alpha_{n}^{0-}=\sum_{v<0} a_{v}^{0}\left(w_{n}\right), & \alpha_{n}^{00}=\sum_{v=0} a_{v}^{0}\left(w_{n}\right), & \alpha_{n}^{0+}=\sum_{v>0} a_{v}^{0}\left(w_{n}\right) ; \\
\alpha_{n}^{+-}=\sum_{v<0} a_{v}^{+}\left(w_{n}\right), & \alpha_{n}^{+0}=\sum_{v=0} a_{v}^{+}\left(w_{n}\right), & \alpha_{n}^{++}=\sum_{v>0} a_{v}^{+}\left(w_{n}\right) .
\end{array}
$$

For example, $\alpha_{n}^{--}$is the number of negative elementary moves which also decreases the energy of the walk, and $\alpha_{n}^{-+}$is the number of negative elementary moves which also increases the energy of the walk. The rest of the $\alpha$ 's are similarly defined.

With these atmospheric statistics defined, a rule needs to be constructed in order to realise the sequence $\phi_{N}$ in equation (22).

The endpoint elementary moves in figures 3 and 4 have the property that no positive elementary move can decrease the energy, and no negative elementary move can increase the energy. Moreover, there are no neutral moves amongst the elementary moves. Thus, assume that

$$
\alpha_{n}^{-+}=0, \quad \alpha_{n}^{+-}=0, \quad \alpha_{n}^{0-}=0, \quad \alpha_{n}^{00}=0 \quad \text { and } \alpha_{n}^{0+}=0 .
$$

The algorithm can be modified appropriately to account for such transitions in models where this is not the case. This assumption leaves the following atmospheric statistics: $\left\{\alpha_{n}^{--}, \alpha_{n}^{-0}, \alpha_{n}^{+0}, \alpha_{n}^{++}\right\}$. That is, distinguish between negative moves which decrease the energy, or negative moves which leave the energy unchanged, or positive moves which leave the energy unchanged, and positive moves which increase the energy.

Introduce parameters $\left\{\beta_{\ell, u}\right\}$ to control positive elementary moves on walks of length $\ell$ and energy $u$, and which leave the energy unchanged (that is, the elementary moves contributing to $\left.\alpha_{n}^{+0}\right)$. Similarly, introduce the parameters $\left\{\gamma_{\ell, u}\right\}$ to control positive elementary moves which increase the energy on walks of length $\ell$ and energy $u$.

The transition probabilities of positive elementary moves which leave the energy unchanged will be proportional to $\beta_{\ell, u}$; if the state has length $\ell$ and energy $u$. For example, since $\ell=16$ and $u=2$ in the walk in figure 2, the transition probability of the positive move in that figure is proporsional to $\beta_{16,2}$. Similarly, the positive elementary move in figure 3 increases the energy; so here the transition probability is proportional to $\gamma_{16,2}$, instead. In figure 4 the positive elementary move also increases the energy, and so its transition probability is proportional to $\gamma_{16,4}$.

Thus, if $w_{n}$ is the current state (of length $\ell_{n}$ and energy $u_{n}$ ), and $w_{n+1}$ is the next state (of length $\ell_{n+1}$ and energy $u_{n+1}$ ), then define the change in length by $\Delta_{n}=\ell_{n+1}-\ell_{n}$, and the change in energy by $\delta_{n}=u_{n+1}-u_{n}$. Notice that $\Delta_{n}= \pm 1$ for endpoint elementary moves, and that $\delta_{n}= \pm 1$, or $\delta_{n}=0$, for the model of adsorbing walks in figures 2 and 3 (but these quantities may take on other values in the model in figure 4). The transition probabilities are chosen such that

$$
P_{r}\left(w_{n} \rightarrow w_{n+1}\right) \propto \begin{cases}\beta_{\ell, u}, & \text { if } \Delta_{n}=+1 \text { and } \delta_{n}=0 \\ 1 & \text { if } \Delta_{n}=-1 \text { and } \delta_{n}=0\end{cases}
$$


where $\ell=\ell_{n}$ and $u=u_{n}$ are functions of $n$. In the case that the energy is changed, then the transition probability is constructed such that

$$
P_{r}\left(w_{n} \rightarrow w_{n+1}\right) \propto \begin{cases}\gamma_{\ell, u}, & \text { if } \Delta_{n}=+1 \text { and } \delta_{n}>0 \\ 1 & \text { if } \Delta_{n}=-1 \text { and } \delta_{n}<0\end{cases}
$$

Normalising the transition probabilities gives

$$
P_{r}\left(w_{n} \rightarrow w_{n+1}\right)= \begin{cases}\frac{\beta_{\ell, u}}{\alpha_{n}^{--}+\alpha_{n}^{-0}+\alpha_{n}^{+0} \beta_{\ell, u}+\alpha_{n}^{++} \gamma_{\ell, u}}, & \text { if } \Delta_{n}=1 \text { and } \delta_{n}=0 ; \\ \frac{\gamma_{\ell, u}}{\alpha_{n}^{--}+\alpha_{n}^{-0}+\alpha_{n}^{+0} \beta_{\ell, u}+\alpha_{n}^{++} \gamma_{\ell, u}}, & \text { if } \Delta_{n}=1 \text { and } \delta_{n}>0 ; \\ \frac{1}{\alpha_{n}^{--}+\alpha_{n}^{-0}+\alpha_{n}^{+0} \beta_{\ell, u}+\alpha_{n}^{++} \gamma_{\ell, u}}, & \text { if } \Delta_{n}=-1 \text { and } \delta_{n} \leq 0 ;\end{cases}
$$

The probability for any type of move can be explicitly computed for any given state $w_{n}$ (of length $\ell$ and energy $u$ ) by computing the $\alpha$ 's. For example, the probability for a positive elementary move which leaves the energy unchanged is $\alpha_{n}^{+0} \beta_{\ell, u} /\left(\alpha_{n}^{--}+\alpha_{n}^{+0} \beta_{\ell, u}+\alpha_{n}^{++} \gamma_{\ell, u}\right)$. For the state on the left in figure 2 this becomes $\beta_{16,2} /\left(1+\beta_{16,2}+\gamma_{16,2}\right)$ since $\alpha_{16}^{--}=1, \alpha_{16}^{+0}=1$ and $\alpha_{16}^{++}=1$.

\subsection{GAS-weights}

Let $w_{0}$ be a starting state (possibly the walk consisting of a single vertex at the origin, of length $\ell_{0}=0$ and energy $u_{0}=0$ ). Implement the endpoint elementary moves on $w_{0}$ by computing its atmosphere recursively and updating it using the transition probabilities in equation (26), starting at $n=0$. This generates a Markov Chain of states in a sequence $\phi_{N}$ (see equation (22)).

Assume that the parameters $\left\{\beta_{n, u}, \gamma_{n, u}\right\}$ are known and fixed, so that the sampling can be implemented by simply computing the transtition probabilities and selecting positive and negative elementary moves with appropriate probabilities.

The probability of the sequence $\phi_{N}$ is given by

$\mathrm{P}_{r}\left(\phi_{N}\right)=\prod_{n=0}^{N-1} \frac{1}{\alpha_{n}^{--}+\alpha_{n}^{-0}+\alpha_{n}^{+0} \beta_{\ell, u}+\alpha_{n}^{++} \gamma_{\ell, u}} \prod_{m}^{\prime} \beta_{\ell, u} \prod_{k}^{\prime \prime} \gamma_{\ell, u}$

where the primed product $\prod^{\prime}$ is over all the $\beta_{\ell, u}$ for transitions through positive elementary moves leaving the energy unchanged, and the double primed product $\Pi^{\prime \prime}$ is over all the $\gamma_{\ell, u}$ where the transition is a positive elementary move increasing the energy. In this expression the $\ell$ and $u$ are functions of $n, m$, and $k$ in each of the products.

A weight $W\left(\phi_{N}\right)$ will be assigned to the sequence $\phi_{N}$. In order to compute the weight, define

$\sigma(j, j+1)=\sigma\left(w_{j} \rightarrow w_{j+1}\right)=\left\{\begin{aligned}-1, & \text { if } \Delta_{j}=+1 \text { and } \delta_{j}=0 \\ +1, & \text { if } \Delta_{j}=-1 \text { and } \delta_{j}=0 \\ 0, & \text { otherwise. }\end{aligned}\right.$ 
Similarly, define

$\rho(j, j+1)=\rho\left(w_{j} \rightarrow w_{j+1}\right)=\left\{\begin{aligned}-1, & \text { if } \Delta_{j}=+1 \text { and } \delta_{j}>0 \\ +1, & \text { if } \Delta_{j}=-1 \text { and } \delta_{j}<0 \\ 0, & \text { otherwise. }\end{aligned}\right.$

That is, the function $\sigma(j, j+1)$ tracks the negative and positive moves along $\phi_{N}$ where the energy is not changed, and the function $\rho(j, j+1)$ tracks the negative and positive transitions along $\phi_{N}$ where the energy is also changed.

Assign the weight

$W\left(\phi_{N}\right)=\left(\frac{\alpha_{0}^{--}+\alpha_{0}^{-0}+\alpha_{0}^{+0} \beta_{0}+\alpha_{0}^{++} \gamma_{0}}{\alpha_{N}^{--}+\alpha_{N}^{-0}+\alpha_{N}^{+0} \beta_{N}+\alpha_{N}^{++} \gamma_{N}}\right) \prod_{j=0}^{N-1} \beta_{\ell, u}^{\sigma(j, j+1)} \prod_{j=0}^{N-1} \gamma_{\ell, u}^{\rho(j, j+1)}$

to the sequence $\phi_{N}$.

The expected value of the weight over sequences of length $N$ from state $w_{0}$ to state $W_{N}$ is

$$
\left\langle W\left(w_{0} \rightarrow w_{N}\right)\right\rangle_{N}=\sum_{\phi: w_{0} \rightarrow w_{N}} P_{r}(\phi) W(\phi) .
$$

Inserting equations (27) and (30) in this, and simplifying, gives

$$
\left\langle W\left(w_{0} \rightarrow w_{N}\right)\right\rangle_{N}=\sum_{\phi: w_{0} \rightarrow w_{N}} \prod_{j=1}^{N} \frac{1}{\alpha_{j}^{--}+\alpha_{j}^{-0}+\alpha_{j}^{+0} \beta_{\ell, u}+\alpha_{j}^{++} \gamma_{\ell, u}} \prod^{n} \beta_{\ell, u} \prod^{n n} \gamma_{\ell, u}
$$

where the product $\prod^{n}$ is over all the $\beta_{\ell, u}$ for transitions through negative elementary moves leaving the energy unchanged, and the product $\prod^{n n}$ is over all $\gamma_{\ell, u}$ where the transition is a negative elementary move decreasing the energy. As before, the $\ell \equiv \ell(j)$ and $u \equiv u(j)$ are functions of $j$ (in other words, functions of the states $w_{j}$ in the sequence $\phi)$.

Reverse all the sequences in equation (32) so that the starting state is $w_{N}$ and the final state is $w_{0}$. Under this reversal all negative elementary moves become positive elementary moves and vice versa. That is, equation (32) becomes

$$
\left\langle W\left(w_{0} \rightarrow w_{N}\right)\right\rangle_{N}=\sum_{\psi: w_{N} \rightarrow w_{0}} \prod_{j=1}^{N} \frac{1}{\alpha_{j}^{--}+\alpha_{j}^{-0}+\alpha_{j}^{+0} \beta_{\ell, u}+\alpha_{j}^{++} \gamma_{\ell, u}} \prod^{n} \beta_{\ell, u} \prod^{n n} \gamma_{\ell, u},
$$

where, as before, the product $\prod^{n}$ is over all the $\beta_{\ell, u}$ for transitions through negative elementary moves along the reverse sequence $\psi$ leaving the energy unchanged, and the product $\prod^{n n}$ is over all $\gamma_{\ell, u}$ where the transition is a negative elementary move along the reverse sequence $\psi$ decreasing the energy.

For example, consider the model of collapsing walks in figure 4 and suppose the sequence $\phi$ is realised, where

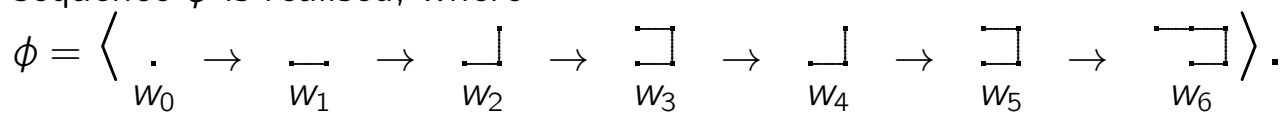

The probability of this sequence is

$\mathrm{P}_{r}(\phi)=\frac{\beta_{0,0}}{4 \beta_{0,0}} \frac{\beta_{1,0}}{\left(1+3 \beta_{1,0}\right)} \frac{\gamma_{2,0}}{\left(1+2 \beta_{2,0}+\gamma_{2,0}\right)} \frac{1}{\left(1+2 \beta_{3,1}\right)} \frac{\gamma_{2,0}}{\left(1+2 \beta_{2,0}+\gamma_{2,0}\right)} \frac{\beta_{3,1}}{\left(1+2 \beta_{3,1}\right)}$ 
The weight of $\phi$ can similarly be computed from equation (30). This gives

$$
W(\phi)=\frac{4 \beta_{0,0}}{1+3 \beta_{4,1}} \times\left(\beta_{0,0}^{-1} \beta_{1,0}^{-1} \gamma_{2,0}^{-1} \gamma_{3,1}^{+1} \gamma_{2,0}^{-1} \beta_{3,1}^{-1}\right) .
$$

The consequence is that

$P_{r}(\phi) W(\phi)=\frac{1}{\left(1+3 \beta_{4,1}\right)} \frac{1}{\left(1+2 \beta_{3,1}\right)} \frac{1}{\left(1+2 \beta_{2,0}+\gamma_{2,0}\right)} \frac{\gamma_{3,1}}{\left(1+2 \beta_{3,1}\right)} \frac{1}{\left(1+2 \beta_{2,0}+\gamma_{2,0}\right)} \frac{1}{\left(1+3 \beta_{1,0}\right)}$,

and this is the probability that a sequence $\psi$, starting in the state $w_{6}$ and terminating in the state $w_{0}$, is realised by the algorithm.

The same observation is true generally for equation (33): The summand is the probability that a particular sequence $\psi$ from state $w_{N}$ to $w_{0}$ is realised by the algorithm, and the summation is over all such sequences $\psi$. That is, $\left\langle W\left(w_{0} \rightarrow w_{N}\right)\right\rangle_{N}=P_{r}\left(w_{N} \rightarrow\right.$ $\left.W_{0}\right)$ is the probability that the algorithm realises a sequence $\psi$ of length $N$ from state $w_{N}$ to state $w_{0}$.

The sequence $\psi$ is a Markov Chain, and if it is aperiodic and irreducible, and if $w_{0}$ is a recurrent state, then asymptotically (for large $N$ ) the probability that the sequence hits the state $w_{0}$ is positive and independent of the starting state $w_{N}$. That is, $P_{r}\left(w_{N} \rightarrow w_{0}\right) \rightarrow C\left(w_{0}\right)>0$ as $N \rightarrow \infty$ where $C\left(w_{0}\right)$ is dependent on the parameters of the algorithm, and independent of $w_{N}$. Thus, the average weight $\left\langle W\left(w_{0} \rightarrow w_{N}\right)\right\rangle_{N} \rightarrow C\left(w_{0}\right)$ as $N \rightarrow \infty$. Summing $\left\langle W\left(w_{0} \rightarrow w_{N}\right)\right\rangle_{N}$ over all the states $w_{N}$ of length $n=\ell\left(w_{N}\right)$ and energy $u$, shows that the average weights of sequences of length $N$ ending in walks of length $n$ and energy $u$ is

$$
\left\langle W_{n, u}\right\rangle_{N}=\sum_{w_{N}: n, u}\left\langle W\left(w_{0} \rightarrow w_{N}\right)\right\rangle_{N} \rightarrow c_{n}(u) C\left(w_{0}\right),
$$

where the summation is over all walks of length $n$ and energy $u$ (and $c_{n}(u)$ is the number of walks of length $n$ and energy $u$ ).

Taking ratios of average weights give

$$
\frac{\left\langle W_{n, u}\right\rangle_{N}}{\left\langle W_{m, v}\right\rangle_{N}} \rightarrow \frac{c_{n}(u)}{c_{m}(v)}
$$

That is, if $c_{n}(u)$ is known for some values of $n$ and $u$, then the ratios of average weights can be used to estimate $c_{m}(v)$.

\subsection{Sampling with GAS}

The algorithm is implemented by choosing a starting state $w_{0}$ and then sampling along a sequence $\phi$ of length $N$. The weight is updated along $\phi$, and collected into bins for walks of length $n$ and energy $u$. Once the sequence is completed, then the average of each bin is computed, giving the average weight $W_{n, u}$ of walks of length $n$ and energy $u$ seen along $\phi$.

This process is repeated $M$ times, so that $M$ sequences of length $N$, denoted by $\left\langle\phi_{1}, \phi_{2}, \ldots, \phi_{M}\right\rangle$ are realised, and for each sequence $\phi_{j}$ the average weight $W_{n, u}^{(j)}$ is calculated. The estimated average weight is computed over the $M$ sequences:

$$
\left[W_{n, u}\right]_{N, M}^{\text {est }}=\frac{1}{M} \sum_{j=1}^{M} W_{n, u}^{(j)}
$$


Table 2: Numerical estimates of $\beta_{n, u}$ for adsorbing walks in $\mathbb{L}_{+}^{2}$

\begin{tabular}{l|lllllllllll}
\hline$n \backslash u$ & 0 & 1 & 2 & 3 & 4 & 5 & 6 & 7 & 8 & 9 & 10 \\
\hline 0 & 1.000 & & & & & & & & & & \\
1 & 0.333 & 1.000 & & & & & & & & & \\
2 & 0.428 & 0.250 & 1.001 & & & & & & & & \\
3 & 0.368 & 0.499 & 0.200 & 1.000 & & & & & & & \\
4 & 0.387 & 0.380 & 0.416 & 0.166 & 1.000 & & & & & \\
5 & 0.373 & 0.396 & 0.427 & 0.429 & 0.142 & 1.000 & & & & & \\
6 & 0.386 & 0.375 & 0.377 & 0.359 & 0.438 & 0.124 & 1.000 & & & & \\
7 & 0.377 & 0.392 & 0.384 & 0.401 & 0.348 & 0.445 & 0.111 & 1.000 & & & \\
8 & 0.383 & 0.378 & 0.388 & 0.356 & 0.368 & 0.327 & 0.449 & 0.100 & 0.999 & & \\
9 & 0.378 & 0.386 & 0.385 & 0.395 & 0.370 & 0.368 & 0.308 & 0.454 & 0.0908 & 0.999 & \\
10 & 0.381 & 0.379 & 0.383 & 0.373 & 0.368 & 0.352 & 0.364 & 0.289 & 0.458 & 0.083 & 0.999 \\
\hline
\end{tabular}

The estimated weight $\left[W_{n, u}\right]_{N, M}^{\text {est }}$ is an estimator of $\left\langle W_{n, u}\right\rangle_{N}$, and so as $M \rightarrow \infty$ and $N \rightarrow \infty$, it is expected that $\left[W_{n, u}\right]_{N, M}^{e s t} \rightarrow c_{n}(u) C\left(W_{0}\right)$. Taking ratios give

$$
\frac{\left[W_{n, u}\right]_{N, M}^{e s t}}{\left[W_{m, v}\right]_{N, M}^{s s t}} \rightarrow \frac{c_{n}(u)}{c_{m}(v)} .
$$

The sampling requires that both $N$ and $M$ are sufficiently large, and there is a tradeoff between these quantities. M should be large enough to have sufficient independent estimates of the weights to compute sound averages, and $N$ should be large enough to have sufficiently long sequences to have sampled large enough regions of state space.

There remains the additional issue of the GAS parameters $\beta_{n, u}$ and $\gamma_{n, u}$. These can be estimated using training runs prior to the simulation. Best results are obtained when the sampling is flat, so that states of size and energy $\{n, u\}$ are sampled uniformly in $\{n, u\}$. This is best achieved when the GAS sequences are random walks on $n$ and $u$. Thus, the probability of a positive move should be, on average, equal to the probability of a negative move. A good choice for $\beta_{n, u}$ is

$$
\beta_{n, u}=\frac{\left\langle\alpha_{n, u}^{-0}\right\rangle}{\left\langle\alpha_{n, u}^{+0}\right\rangle}
$$

where $\left\langle\alpha_{n, u}^{-0}\right\rangle$ is the average negative atmosphere which does not decrease the energy, and $\left\langle\alpha_{n, u}^{+0}\right\rangle$ is the average positive atmosphere which does not increase the energy, of walks of length $n$ and energy $u$. 
Table 3: Numerical estimates of $\gamma_{n, u}$ for adsorbing walks in $\mathbb{L}_{+}^{2}$

\begin{tabular}{|c|c|c|c|c|c|c|c|c|c|c|c|}
\hline$n \backslash u$ & 0 & 1 & 2 & 3 & 4 & 5 & 6 & 7 & 8 & 9 & 10 \\
\hline 0 & 0. 499 & & & & & & & & & & \\
\hline 1 & 0. 499 & 1. 000 & & & & & & & & & \\
\hline 2 & 0. 374 & 1. 001 & 0. 999 & & & & & & & & \\
\hline 3 & 0. 436 & 0. 801 & 0. 998 & 0. 999 & & & & & & & \\
\hline 4 & 0. 451 & 0. 668 & 0. 832 & 0. 998 & 1. 000 & & & & & & \\
\hline 5 & 0. 461 & 0. 751 & 0.857 & 0.855 & 1. 000 & 0. 999 & & & & & \\
\hline 6 & 0. 463 & 0.716 & 0. 721 & 0.873 & 0. 874 & 0. 999 & 0. 999 & & & & \\
\hline 7 & 0. 470 & 0. 734 & 0. 767 & 0. 845 & 0. 887 & 0. 889 & 0. 998 & 1. 000 & & & \\
\hline 8 & 0. 470 & 0. 728 & 0. 709 & 0. 775 & 0.834 & 0. 898 & 0. 899 & 0. 999 & 1. 00 & & \\
\hline 9 & 0. 473 & 0. 741 & 0. 721 & 0. 807 & 0.835 & 0.845 & 0. 908 & 0. 907 & 1. 000 & 0. 999 & \\
\hline 10 & 0. 474 & 0. 736 & 0. 699 & 0. 752 & 0. 795 & 0. 836 & 0. 853 & 0. 915 & 0. 915 & 1. 000 & 0. 999 \\
\hline
\end{tabular}

A similar argument shows that a good choice for $\gamma_{n, u}$ is

$$
\gamma_{n, u}=\frac{\left\langle\alpha_{n, u}^{--}\right\rangle}{\left\langle\alpha_{n, u}^{++}\right\rangle}
$$

where $\left\langle\alpha_{n, u}^{--}\right\rangle$is the average negative atmosphere which decreases the energy, and $\left\langle\alpha_{n, u}^{++}\right\rangle$ is the average positive atmosphere which increases the energy, of walks of length $n$ and energy $u$.

Computed values for $\beta_{n, u}$ and $\gamma_{n, u}$ for a square lattice adsorbing walk model in figure 2 are given in tables 2 and 3 for $0 \leq n \leq 10$ and $0 \leq u \leq 10$. These data are chopped at an accuracy of three decimal places.

Observe that $\beta_{n, n}=1$ (when rounded) and $\gamma_{n, n}=1$. This is expected, since there are exactly two states if $n>0$ and $n=u$ (a completely adsorbed walk which never leaves the adsorbing line).

With these values of $\beta_{n, u}$ and $\gamma_{n, u}$ the sampling is reasonably flat, as shown in table 4. The data in table 4 is the number of times a sequence of length $10^{6}$ visited states of length $n$ and energy $u$. The maximum length was set at $n=500$, so that the number of pairs $(n, u)$ is 124750; thus, the expected number of visits to states of length $n$ and energy $u$ is roughly 8000 . The data in table 4 are spread around this number, and the distribution is a reasonably flat histogram. There are exceptions for data along the diagonal where a larger number is seen. The explanation for this is that the sequence can only visit states along the diagonal if it starts in $(n, u)=(0,0)$ and then stay on the diagonal (doing a random walk on states of length and energy both equal to $n$ ) - 
Table 4: The frequency of states sampled to $n=10$ and $u=10$ for adsorbing walks in $\mathbb{L}_{+}^{2}$

\begin{tabular}{l|lllllllllll}
\hline$n \backslash u$ & 0 & 1 & 2 & 3 & 4 & 5 & 6 & 7 & 8 & 9 & 10 \\
\hline 0 & 8658 & & & & & & & & & & \\
1 & 8616 & 13208 & & & & & & & & & \\
2 & 9642 & 7711 & 13261 & & & & & & & & \\
3 & 8971 & 9622 & 7144 & 13012 & & & & & & \\
4 & 8919 & 9576 & 9261 & 6528 & 12883 & & & & & \\
5 & 8957 & 8947 & 9501 & 9388 & 5998 & 13072 & & & & & \\
6 & 8982 & 8736 & 9220 & 9019 & 9281 & 6055 & 13263 & & & & \\
7 & 9059 & 8562 & 9026 & 9095 & 8711 & 9733 & 5812 & 13278 & & & \\
8 & 8996 & 8398 & 9030 & 8844 & 8568 & 8914 & 9493 & 5728 & 13362 & & \\
9 & 9169 & 8162 & 8788 & 8772 & 8579 & 8843 & 8417 & 9689 & 5678 & 13204 & \\
10 & 9134 & 8182 & 8580 & 8434 & 8559 & 8898 & 8390 & 8221 & 10101 & 5477 & 12883 \\
\hline
\end{tabular}

the elementary moves chosen in the simulation do not include neutral moves, and so the sequence cannot enter the diagonal except at $n=0$.

Observe that since $c_{n}^{+}(n)=2$ for $n>0$ in this model, that these states are rare, but are sampled frequently by the algorithm. This is an example of rare event sampling, where an algorithm spends significant time sampling rare states in the tails of a distribution in order to get good estimates of microcanonical quantities.

Simulations were performed by collecting data over 500 realised sequences, each of length $10^{9}$. Numerical estimates of $c_{n, u}$ are shown in table 5 . The data in table 5 are rounded to the nearest integer. These data can be compared to exact counts to verify the algorithm and its implementation.

Partition functions (see equation (6)) can be directly estimated from the microcanonical data in table 5, for example, for $n=6$ the partition function is approximated by

$$
Z_{6}(a)=131+106 a+56 a^{2}+28 a^{3}+14 a^{4}+2 a^{5}+2 a^{6}
$$

where $a=e^{v / k T}$ is a Boltzmann factor ( $v$ is the interaction energy associated with a single visit).

Additional data were collected in the square and cubic lattices. For example, the average mean square radius of gyration of walks of length $n$ and energy $u$ were also determined, as was the average height of the endpoint of the walks. In addition, data were also collected on the average positive and negative atmospheric statistics from which estimates of the $\beta_{n, u}$ and $\gamma_{n, u}$ were made (see equations (41) and (42)). 
Table 5: Estimates of $c_{n}^{+}(u)$ to $n=10$ and $u=10$ for adsorbing walks in $\mathbb{L}_{+}^{2}$

\begin{tabular}{l|lllllllllll}
\hline$n \backslash u$ & 0 & 1 & 2 & 3 & 4 & 5 & 6 & 7 & 8 & 9 & 10 \\
\hline 0 & 1 & & & & & & & & & & \\
1 & 1 & 2 & & & & & & & & & \\
2 & 3 & 2 & 2 & & & & & & & & \\
3 & 7 & 8 & 2 & 2 & & & & & & & \\
4 & 19 & 16 & 10 & 2 & 2 & & & & & & \\
5 & 49 & 42 & 24 & 12 & 2 & 2 & & & & & \\
6 & 131 & 106 & 56 & 28 & 14 & 2 & 2 & & & & \\
7 & 339 & 283 & 148 & 76 & 32 & 16 & 2 & 2 & & & \\
8 & 897 & 720 & 385 & 193 & 92 & 36 & 18 & 2 & 2 & & \\
9 & 2338 & 1905 & 990 & 543 & 249 & 110 & 40 & 20 & 2 & 2 & \\
10 & 6178 & 4932 & 2571 & 1372 & 672 & 298 & 130 & 44 & 22 & 2 & 2 \\
\hline
\end{tabular}

\section{Numerical Results}

\subsection{Adsorbing walks in the square lattice}

The finite size free energy $\mathcal{F}_{n}(a)$ was determined from the data and is plotted in figure 5 for walks of lengths $n=50 \mathrm{~N}$ with $=1,2,3, \ldots, 10 . \mathcal{F}_{n}(a)$ is a function of the combined variable $\tau=n^{1 / 2}\left(a-a_{c}^{+}\right)$(see equations 110$)$ and $(12)$, and note that the crossover exponent is $\left.\phi=\frac{1}{2}\right)$. Plotting the free energy $\mathcal{F}_{n}(a)$ against $\tau$ should collapse the data to a single underlying curve (near the critical point $a_{c}^{+}$; that is, for small values of $\tau$ ) which exposes the scaling function $g$ in equation (12). This is displayed in figure 6 .

The energy $\mathcal{E}_{n}(a)$ and specific heat $\mathcal{C}_{n}(a)$ (see equation (13)) can be determined by differentiation of $\mathcal{F}_{n}(a)$ and is plotted in figure 7 against log $a$ and in figure 8 against $\tau$. These curves clearly show the adsorption transition in the model at a critical value of $a$.

Closer inspection of the specific heat curves in figure 7 shows that they intersect each other close to a fixed point. To the left of this point the curves decrease with increasing $n$ to zero, and to the right of this point the curves increase with increasing $n$. The common point of intersection is located approximatedly at the critical adsorption point $a_{c}^{+}$in the model. In figure 9 the specific heat curves are magnified in a region close to the point where they intersect.

3.1.1. Location of the critical point $a_{c}^{+}$: In order to determine $a_{c}^{+}$, consider the finite size energy density $\mathcal{E}_{n}(a)=a \frac{d}{d a} \mathcal{F}(a)$ per edge in the model. It is known that (see, for 


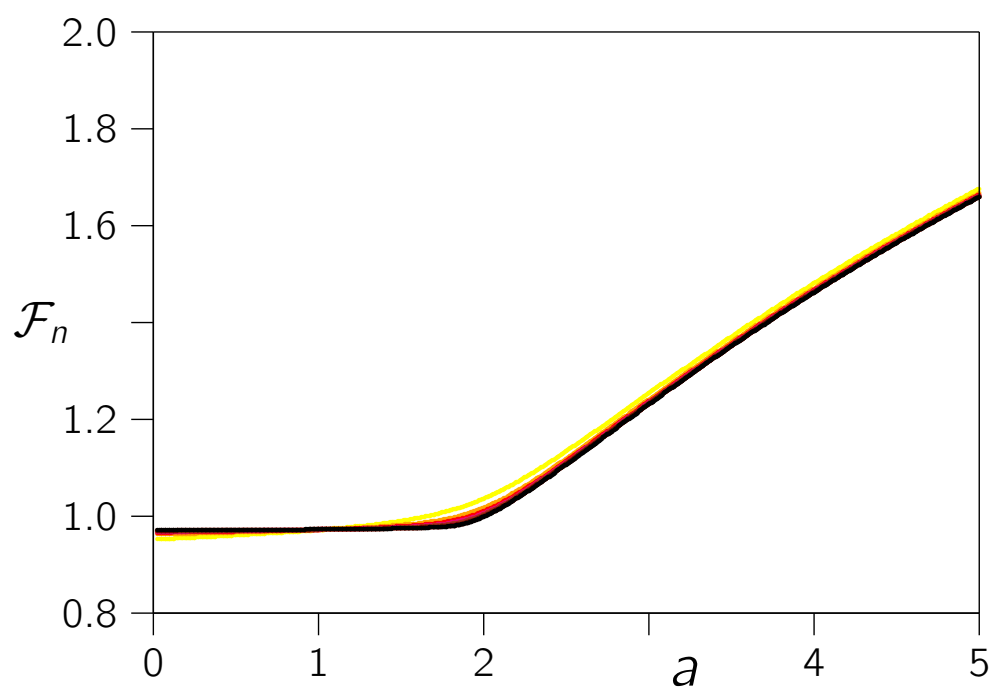

Figure 5: The finite size free energy $\mathcal{F}_{n}(a)$ of adsorbing positive walks as a function of a for adsorbing walks in $\mathbb{L}_{2}^{+}$. The lengths of the walks varied from $n=50$ (yellow) with colours increasing in hue to black when $n=500$ in steps of 50 . For small values of a $\mathcal{F}_{n}(a)$ converges to log $\mu_{2}$, but for a large $\mathcal{F}_{n}(a)>\log \mu_{2}$.

example, equation (13) and reference [23])

$$
\mathcal{E}_{n}(a)=n^{\phi-1} h_{n}(\tau)
$$

where $h_{n}$ is a scaling function. From this equation, construct the ratio

$$
\frac{\log \left(n \mathcal{E}_{n}(a)\right)}{\log \left(m \mathcal{E}_{m}(a)\right)}=\frac{\phi \log n+\log h_{n}(\tau)}{\phi \log m+\log h_{m}(\tau)}
$$

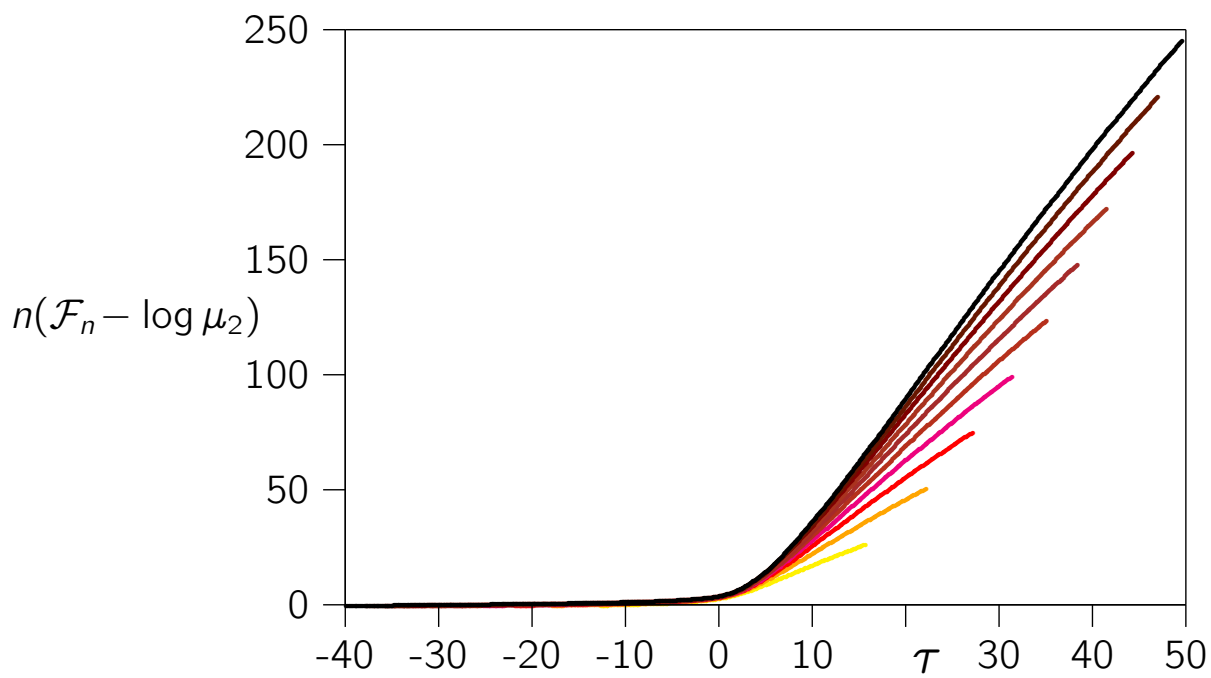

Figure 6: The scaled free energy $n\left(\mathcal{F}_{n}-\log \mu_{2}\right)$ as a function of $\tau=n^{1 / 2}\left(a-a_{c}^{+}\right)$, where $a_{c}^{+}=1.78$, for adsorbing walks in $\mathbb{L}_{2}^{+}$. The lengths of the walks varied from $n=50$ (yellow) with colours increasing in hue to black when $n=500$ in steps of 50. The data collapse for small $|\tau|$ (this is the critical scaling regime), and will approach a limiting curve as $n \rightarrow \infty$, also for large $\tau$, given by the limiting free energy. 

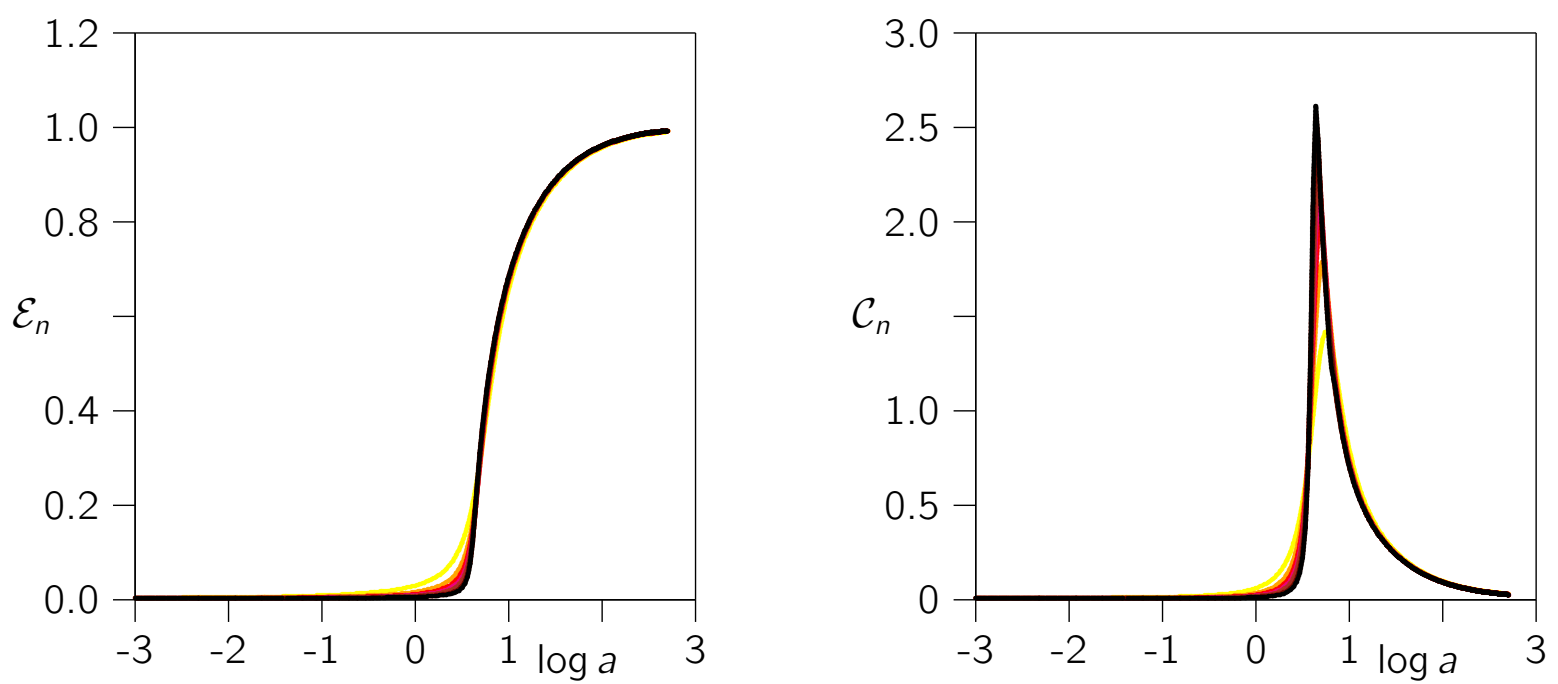

Figure 7: The energy $\mathcal{E}_{n}(a)$ and specific heat $\mathcal{C}_{n}(a)$ (see equation (13)) as a function of log a for adsorbing walks in $\mathbb{L}_{2}^{+}$. The lengths of the walks varied from $n=50$ (yellow) with colours increasing in hue to black when $n=500$ in steps of 50 .

If $a=a_{c}^{+}$, then $\tau=0$, and the above simplifies to

$$
\frac{\log \left(n \mathcal{E}_{n}\left(a_{c}^{+}\right)\right)}{\log \left(m \mathcal{E}_{m}\left(a_{c}^{+}\right)\right)}=\frac{\phi \log n+\log h_{n}(0)}{\phi \log m+\log h_{m}(0)} .
$$

For large values of $n$ and $m, \log h_{n}(0)$ and $\log h_{m}(0)$ approaches the same constant $C$, so that the above becomes

$$
\frac{\log \left(n \mathcal{E}_{n}\left(a_{c}^{+}\right)\right)}{\log \left(m \mathcal{E}_{m}\left(a_{c}^{+}\right)\right)}=\frac{\phi \log n+C}{\phi \log m+C}+\text { small correction. }
$$
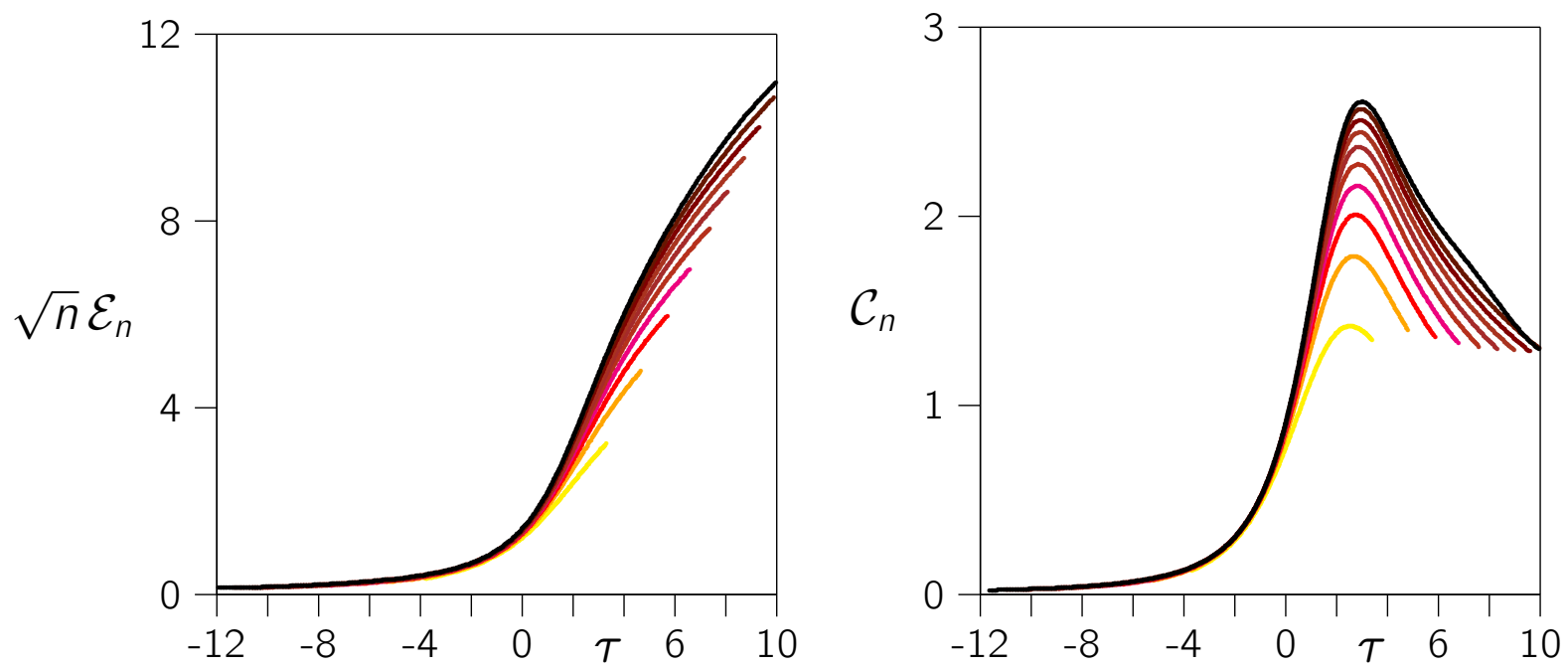

Figure 8: The rescaled energy density (left) and the specific heat (right) plotted as a function of $\tau=n^{1 / 2}\left(a-a_{c}^{+}\right)$near the critical point $a_{c}^{+}$for adsorbing walks in $\mathbb{L}_{2}^{+}$. The curves approach a limiting curve with increasing $n$. The lengths of the walks varied from $n=50$ (yellow) with colours increasing in hue to black when $n=500$ in steps of 50 . The data collapse for small values of $|\tau|$ (this is the critical scaling regime which contains the critical point). For larger values of $|\tau|$ the curves will approach a limiting curve as $n \rightarrow \infty$ given by the limiting free energy. 


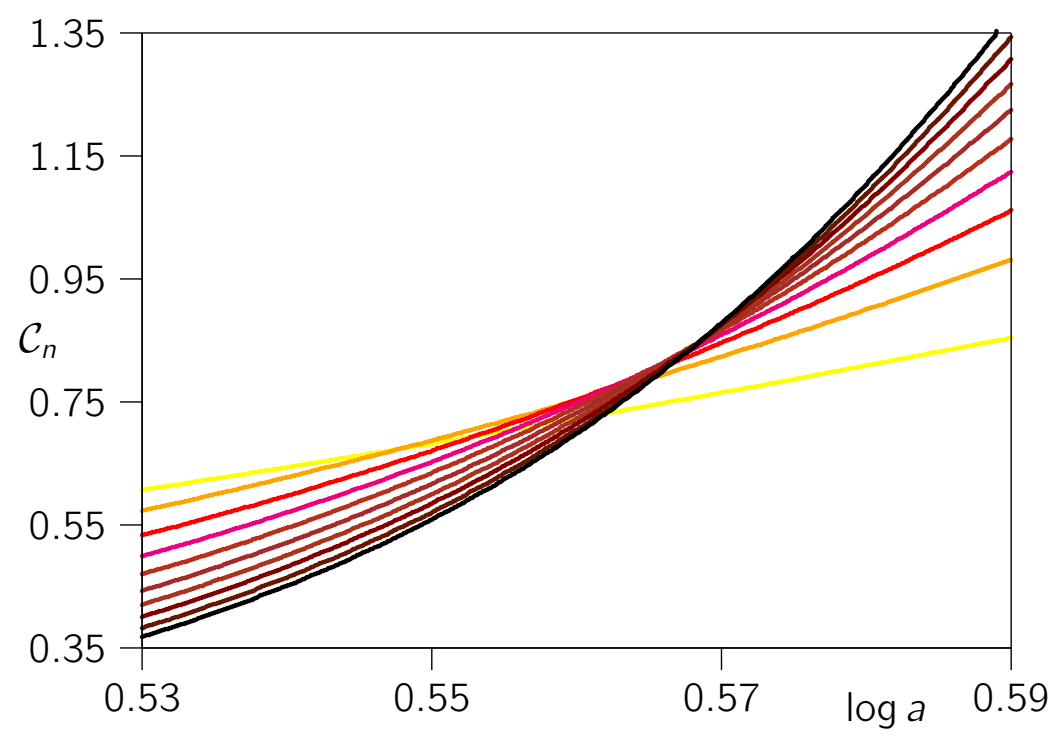

Figure 9: A magnification of the point close to where the specific heat curves in figure 7 intersect, for adsorbing walks in $\mathbb{L}_{2}^{+}$. The lengths of the walks varied from $n=50$ (yellow) with colours increasing in hue to black when $n=500$ in steps of 50 .

When $C$ is small compared to $\log m$, the right hand side may be expanded to obtain

$$
\frac{\log \left(n \mathcal{E}_{n}\left(a_{c}^{+}\right)\right)}{\log \left(m \mathcal{E}_{m}\left(a_{c}^{+}\right)\right)}=\frac{\log n}{\log m}+\left[\frac{C}{\phi \log m}-\frac{C \log n}{\log ^{2} m}\right]-\frac{C^{2}}{\phi^{2} \log ^{2} m}+\text { small correction. }
$$

The signs of the two terms in square brackets are opposite, and for $n$ and $m$ not too far apart, these terms grow about at the same rate as the last term $\frac{C^{2}}{\phi^{2} \log ^{2} m}$. That is, the approximation

$$
\frac{\log \left(n \mathcal{E}_{n}\left(a_{c}^{+}\right)\right)}{\log \left(m \mathcal{E}_{m}\left(a_{c}^{+}\right)\right)}=\frac{\log n}{\log m}-\frac{C_{0}}{\phi^{2} \log ^{2} m}+\text { small correction, }
$$

where $C_{0}$ is a constant, should be accurate at the critical point. Divide both sides by $\frac{\log n}{\log m}$ to obtain

$$
P_{n, m}\left(a_{c}^{+}\right)=\frac{\log \left(n \mathcal{E}_{n}\left(a_{c}^{+}\right)\right)}{\log \left(m \mathcal{E}_{m}\left(a_{c}^{+}\right)\right)} \frac{\log m}{\log n}=1-\frac{C^{2}}{\phi^{2} \log n \log m}+\text { small correction, }
$$

Solving for the critical point by inverting $P_{n, m}$ gives the solution $a_{n, m}^{+}$as an approximation of the critical point, namely $a_{n, m}^{+}=P_{n, m}^{-1}\left(1-\frac{c^{2}}{\phi^{2} \log n \log m}+\right.$ small correction $)$ which may be expanded to

$$
a_{n, m}^{+}=P_{n, m}^{-1}(1)-\frac{C_{1}}{\log n \log m}+\text { small correction, }
$$

where $C_{1}$ is a constant. That is, for given values of $n$ and $m$, an estimate of $a_{c}^{+}$can be obtained by determining the solution of $P_{n, m}(a)=1$, or $a_{n, m}^{+}$is estimated obtained by solving for $a$ in

$$
\frac{\log \left(n \mathcal{E}_{n}(a)\right)}{\log \left(m \mathcal{E}_{m}(a)\right)} \frac{\log m}{\log n}=1
$$




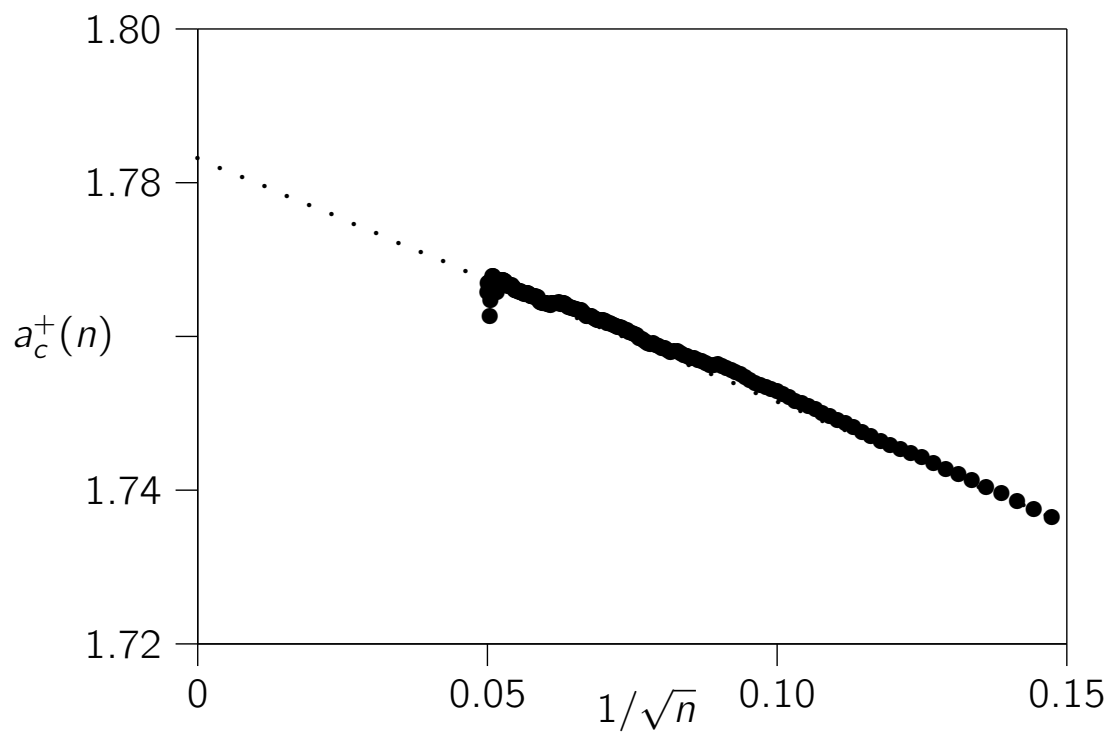

Figure 10: Extrapolating the intersections between specific heat curves for adsorbing walks in $\mathbb{L}_{2}^{+}$. These data points are the intersections of the specific heat for $n$ and $n+100$, for $n$ even and $n \in[44,400]$. The data (except for some data points at the largest values of $n$ ) line up along a line if plotted against $1 / \sqrt{n}$. The location of the critical point can be estimated by extrapolating the line to the left vertical axis; this gives the estimate in equation (55).

In the above, $m$ was put equal to $n-100$, and $n$ was assigned values starting at $n=200$ to $n=500$ in steps of 1 . The estimates $a_{n}^{+} \equiv a_{n, n-100}^{+}$showed no systematic dependence on $n$, and a simple average over all $n \in[200,500]$ gives the best value

$$
a_{c}^{+}=1.7788 \pm 0.0029 \text {. }
$$

The confidence interval is obtained by doubling the square root of the variance of the estimates $a_{n}^{+}$. This result compares well with the result in reference [3], namely $a_{c}^{+}=1.77564$ (obtained by the exact enumeration of adsorbing walks), and also with $a_{c}^{+}=1.759 \pm 0.018$ in reference [23] (obtained by using a Multiple Markov Chain implementation of the Berretti-Sokal algorithm [6]).

The estimate (53) can be used to determine the crossover exponent $\phi$. By equation (13), the specific heat scales as $\mathcal{C}_{n}\left(a_{c}^{+}\right) \sim n^{\alpha \phi} h_{c}(0)$ when $a=a_{c}^{+}$. That is, an estimate of $\alpha \phi$ is obtained by computing $\frac{\log \mathcal{C}_{n}\left(a_{c}^{+}\right)}{\log n}$. Computing this for $100 \leq n \leq 500$, and taking the mean as the best estimate (and estimating a confidence interval by doubling the square root of the variance of the estimates), gives $\alpha \phi=-0.0091 \pm 0.0162$. Determining $\phi$ by using equation (11) then gives the best estimate for $\phi$ :

$$
\phi=0.4955 \pm 0.0081 \text {. }
$$

This result is consistent with $\phi=\frac{1}{2}[2,4,9]$, and compares well with other estimates in the literature (for example, $\phi=0.501 \pm 0.014$ in reference [23]).

3.1.2. The critical point $a_{c}^{+}$and the specific heat $\mathcal{C}_{n}(a)$ : The best estimates above may be compard to estimates obtained from the specific heat curves in figure 7 . These curves intersect each other near $a_{c}^{+}$, and the region containing the intersections (in figure 9 ) is magnified in figure 10 . 
In general the location of the intersection between $\mathcal{C}_{n}(a)$ and $\mathcal{C}_{m}(a)$ is a function of $n$ and $m$. The location of the critical point $a_{c}^{+}$can be estimated by extrapolating this dependence. Consider for example the intersections between the curves $\mathcal{C}_{n}(a)$ and $\mathcal{C}_{n+100}(a)$. The location of these intersections are plotted against $\frac{1}{\sqrt{n}}$ in figure 10 , where $n=2 N$ and $N \in[23,200]$. The data lie along a straight line, except for a few points at the largest values of $n$ (where the data is more uncertain). The best line through the data can be extrapolated to its intersection with the vertical axis (where $\frac{1}{\sqrt{n}}=0$ ). This gives an estimate of $a_{c}^{+}$as being located in the interval $[1.77,1.79]$. Using a linear least squares model for $n \geq 10$ gives the (extrapolated) estimate $a_{c}^{+} \approx 1.7839$. By examining the spread of the data in figure 10, a confidence interval can be estimated. The result is

$$
a_{c}^{+}=1.784 \pm 0.010 \text {. }
$$

This estimate is consistent with the best estimate in equation (53).

An alternative approach to determining the critical point $a_{c}^{+}$, is to consider the scaling of the specific heat in equation (13). Taking ratios for $n$ and $m$, and then logarithms, give

$$
\log \left(\frac{\mathcal{C}_{n}(a)}{\mathcal{C}_{m}(a)}\right)=\alpha \phi \log \left(\frac{n}{m}\right)+\log \left(\frac{h_{c}\left(n^{\phi}\left(a-a_{c}^{+}\right)\right)}{h_{c}\left(m^{\phi}\left(a-a_{c}^{+}\right)\right)}\right) .
$$

Observe that the last term is zero when $a=a_{c}^{+}$. Since, in addition, $\alpha=2-\frac{1}{\phi}=0$ in this model, this shows that an estimate of the critical point is given by the solution of

$$
\log \left(\frac{\mathcal{C}_{n}(a)}{\mathcal{C}_{m}(a)}\right)=0
$$

Solving this for $150 \leq n \leq 500$ (and $n$ a multiple of 10), and for $m=n-k$ where $k \in\{10,20, \ldots, 100\}$ gives a large collection of estimates of $a_{c}^{+}$, with mean

$$
a_{c}^{+}=1.762 \pm 0.016
$$

The confidence interval is one-half of difference between the maximum and minimum estimates of $a_{c}^{+}$. This estimate is slightly less than, but still consistent with, the results in equation (53) and equation (55). Recall that it is also predicated on the assumption that $\alpha=0\left(\right.$ or $\left.\phi=\frac{1}{2}\right)$.

3.1.3. The microcanonical density function: The microcanonical density function of visits in adsorbing positive walks is determined from the microcanonical data in the model, and is given by

$$
P^{+}(\epsilon)=\lim _{n \rightarrow \infty}\left(c_{n}^{+}(\lfloor\epsilon n\rfloor)\right)^{1 / n}=\lim _{n \rightarrow \infty} P_{n}^{+}(\epsilon),
$$

where $P_{n}^{+}(\epsilon)=\left(c_{n}^{+}(\lfloor\epsilon n\rfloor)\right)^{1 / n}$ is a finite size approximation to $P^{+}(\epsilon)$. Existence of the limit is known (see for example reference [22]), and $\log P^{+}(\epsilon)$ is a concave function of $\epsilon$.

$P^{+}(\epsilon)$ can be estimated by interpolating the finite size approximations $P_{n}^{+}(\epsilon)$ and then extrapolating to $n=\infty$ by fitting a least squares model to the data. In figure 11 the data for the extrapolated function $P^{+}(\epsilon)$ is plotted together with $P_{n}^{+}(\epsilon)$ for $n=100$ and $n=500$. 


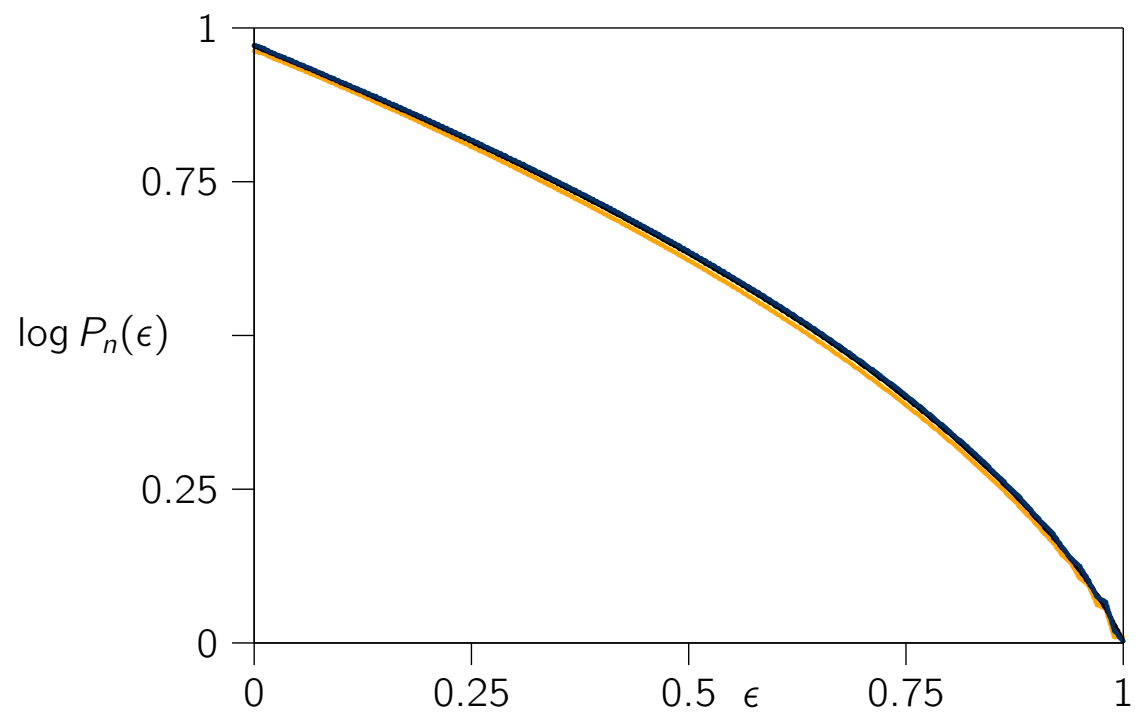

Figure 11: The microcanonical density function $P^{+}(\epsilon)$ of visits of adsorbing square lattice walks. These data the finite size approximations $P_{n}^{+}(\epsilon)$ to $P^{+}(\epsilon)$ for $n=100$ and $n=500$, as well as the extrapolated estimate of $P^{+}(\epsilon)$. The right derivative of $\log P^{+}(\epsilon)$ at $\epsilon=0$ is an estimate of the location of the critical adsorption point $a_{c}^{+}$in the model (see, for example, reference [22]).

A least squares fit of a quadratic to $\log P^{+}(\epsilon)$ for $\epsilon \in[0,0.1]$ gives the $\log P^{+}(\epsilon) \approx$ $0.97007-0.58190 \epsilon-0.13030 \epsilon^{2}$, and by taking the right derivative and then taking $\epsilon \rightarrow 0^{+}$, an estimate for the critical point is obtained:

$$
a_{c}^{+} \approx 1.789 \text {. }
$$

This is close to the estimates obtained in equations (53) and (55), showing consistency in the data and the analysis above.

The free energy $\mathcal{F}(a)$ is the Legendre transform of $\log P^{+}(\epsilon)$. This may be estimated by fitting a polynomial to $\log P^{+}(\epsilon)$. If a cubic polynomial in $\epsilon$ is fitted to $\log P^{+}(\epsilon)$ for $0 \leq \epsilon \leq 0.5$, then the estimated free energy for $a>a_{c}^{+}$is approximately

$F(a) \approx 1.0376-0.1153 \log a-(0.3306-0.5693 \log a) \sqrt{-1.3603+2.3422 \log a}$.

The critical point can be estimated as that location where the square root in the above is zero. This gives $a_{c}^{+} \approx 1.787$. Similarly, the factor $(0.3306-0.5693 \log a)$ vanishes when $a_{c}^{+} \approx 1.787$.

3.1.4. Metric data: The mean square radius of gyration $R_{n}^{2}$, and the mean height $H_{n}$ of the endpoint of the walk, are functions of the adsorption activity $a$. In the desorbed phase (for $a<a_{c}^{+}$) it is expected that $R_{n}^{2} \sim n^{2 \nu}$, and $H_{n} \sim n^{\nu}$, where $\nu=\frac{3}{4}$ is the metric exponent [14]. This scaling changes in the adsorbed phase (when $a>a_{c}^{+}$) to $R_{n}^{2} \sim n^{2}$ and $H_{n} \sim$ constant. These expectations are confirmed by the data, as seen, for example, in figure 12, where data for the mean square radius of gyration are normalised and then plotted as a function of a. These graphs clearly show two scaling regimes, namely a high temperature phase (when $a<a_{c}^{+}$) where the walk has bulk critical exponents and is desorbed, and a low temperature phase where the walk stays near the adsorbing boundary and has critical exponents of a linear object. 

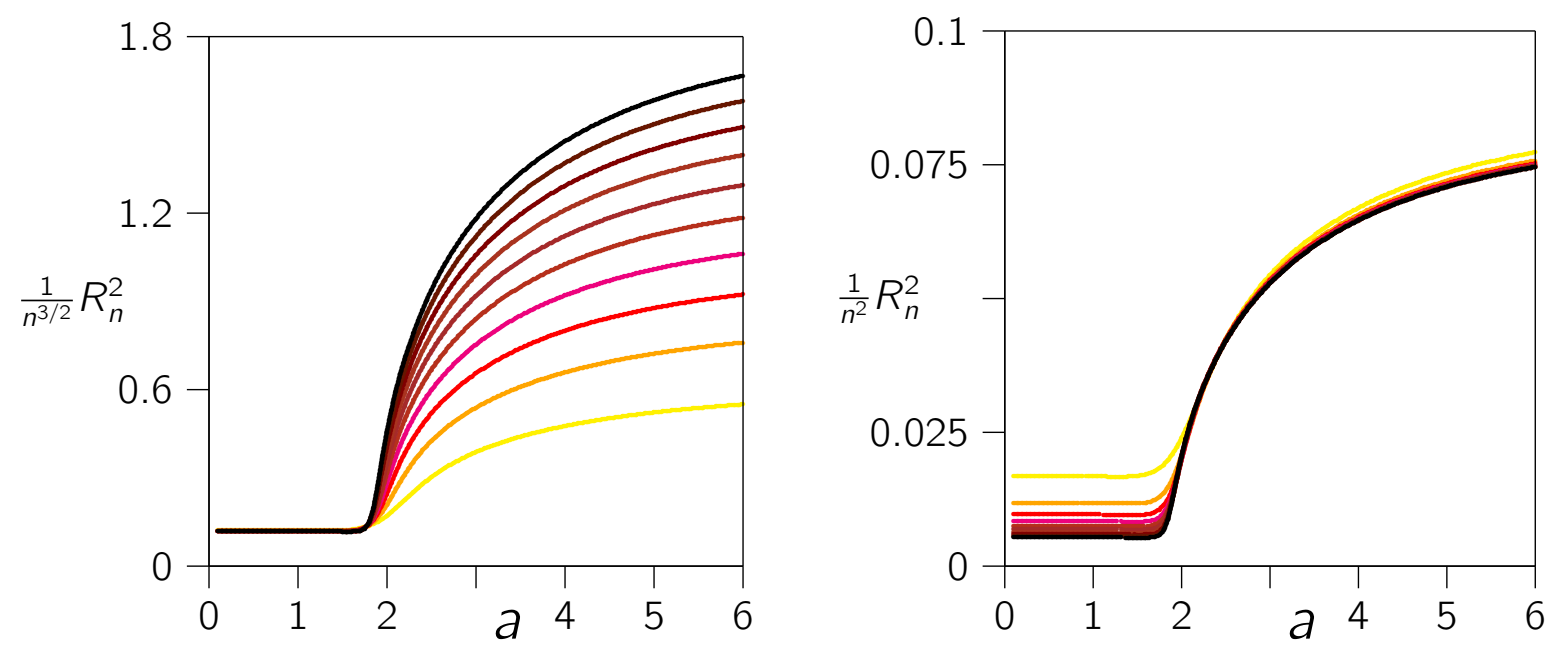

Figure 12: The mean square radius of gyration as a function of $a$, for adsorbing walks in $\mathbb{L}_{2}^{+}$. In the left panel $R_{n}^{2}$ is divided by $n^{2 \nu}$. For $a<a_{c}^{+}$this shows that $R_{n}^{2} \sim n^{2 \nu}$, and for $a>a_{c}^{+} R_{n}^{2}$ increases in size faster than $n^{2 \nu}$ (since it is adsorbed, it stays in the vicinity of the adsorbing boundary, and $R_{n}^{2} \sim n^{2}$ in this regime. This is seen in the right panel, where $R_{n}^{2}$ is divided by $n^{2}$. For $a>a_{c}^{+}$the curves collapse to a single underlying function, exposing the scaling in the adsorbed phase. The lengths of the walks varied from $n=50$ (yellow) with colours increasing in hue to black when $n=500$ in steps of 50 .

In general, the metric exponent associated with $R_{n}^{2}$ is a function of $a$, and it will be denoted by $\nu_{a}$, where $\nu_{a}=\frac{3}{4}$ in the desorbed phase, and $\nu_{a}=1$ in the adsorbed phase. This exponent may be estimated from the mean square radius of gyration $R_{n}^{2}$ data by examining the ratio

$$
2 \nu_{n, m}(a)=\frac{\log \left(R_{n}^{2} / R_{m}^{2}\right)}{\log (n / m)}
$$

Here, $\nu_{n, m}(a)$ is a function of $n$ and $m$. By averaging over $m$, the estimate $\nu_{n}(a)=$ $\left\langle\nu_{n, m}(a)\right\rangle_{m}$ may be determined. In particular, fixing $n$ and taking the average over $m$ for $100 \leq m \leq 500$ in multiples of 5 (and for $m \neq n$ ) gives estimates of $\nu_{n}(a)$. These results are plotted in figure 13 for $n \in\{50,100,150, \ldots, 500\}$. The data for $a \leq 1.5$ gives $\nu \approx 0.747$, and for $a \geq 1.95, \nu \approx 1.01$. These results are evidence for the exact value $\nu_{a}=\frac{3}{4}$ in the desorbed phase, and $\nu_{a}=1$ in the adsorbed phase.

The function $\nu_{n}(a)$ should scale with the combined variable $\tau=n^{\phi}\left(a-a_{c}^{+}\right)$. That is, one may expect that $\nu_{n}(a)=\nu(\tau)$, where $\nu$ is a scaling function. In figure 14 the data in figure 13 are rescaled by plotting against $\tau$ to uncover the scaling function $\nu$.

The (normalised) average height of the endpoint of the walk is plotted as a function of $a$ in figure 15. These data show a clear transition where the scaling of $H_{n}$ changes. That is, the metric exponent associated with $H_{n}$ is a function of $a$, and is denoted by $\nu_{a}^{\perp}$, where $\nu_{a}^{\perp}=\nu=\frac{3}{4}$ if $a<a_{c}^{+}$, and $\nu_{a}^{\perp}=0$ if $a>a_{c}^{+}$, so that $H_{n} \sim n^{\nu \frac{\perp}{a}}$. The graph of $n^{-3 / 4} H_{n}$ contains a set of curves which decreases with increasing $a$. These curves intersect each other close to $a_{c}^{+}$.

An approach similar to the ratio method in equation (62) may be used to estimate $\nu_{a}^{\perp}$ (which will be referred to as the vertical metric exponent):

$$
\nu_{n, m}^{\perp}(a)=\frac{\log \left(H_{n} / H_{m}\right)}{\log (n / m)} .
$$




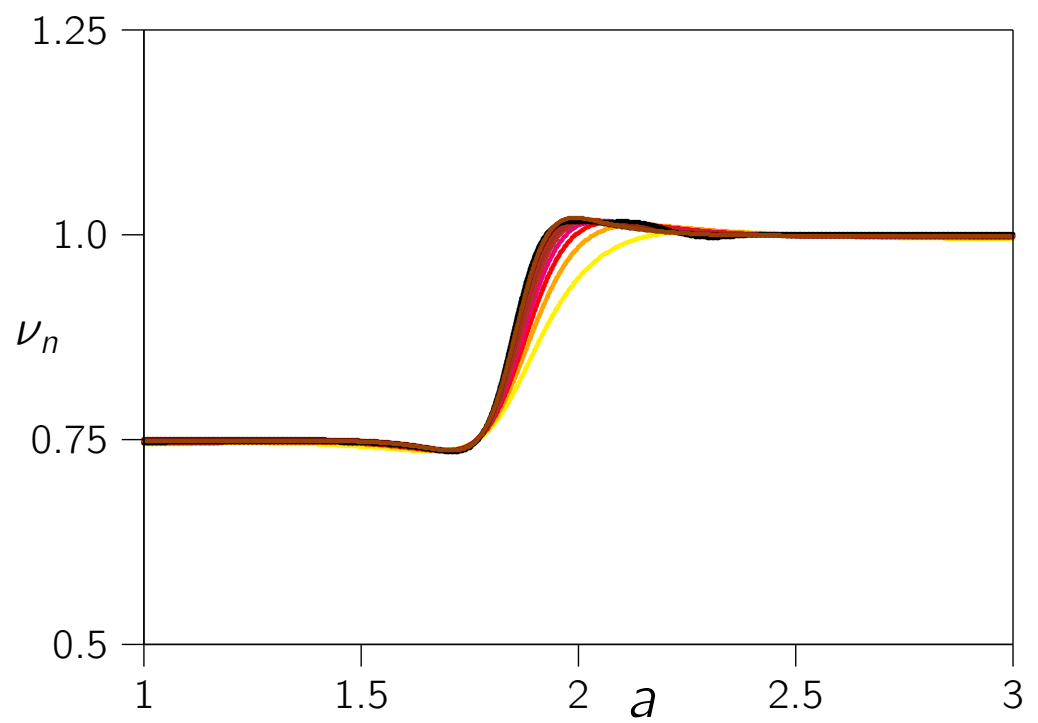

Figure 13: The metric exponent as a function of a, for adsorbing walks in $\mathbb{L}_{2}^{+}$. The exponent was estimated from equation (62) by fixing $n$ and then averaging over $m$ for $100 \leq m \leq 500$. With increasing $n$ the change from $\nu=\frac{3}{4}$ in the desorbed phase, to $\nu=1$ in the adsorbed phase will become a step function at $a=a_{c}^{+}$. The lengths of the walks varied from $n=50$ (yellow) with colours increasing in hue to black when $n=500$ in steps of 50 .

This estimate of $\nu_{a}^{\perp}$ is a function of $n$ and $m$, and may be averaged over $m$ to obtain $\nu_{n}^{\perp}(a)=\left\langle\nu_{n, m}(a)\right\rangle_{n}$. Taking the average for $100 \leq m \leq 500$ in multiples of 5 to estimate $\nu_{n}^{\perp}(a)$ gives the curves in figure 16 when plotted against the combined variable $\tau=n^{\phi}\left(a-a_{c}^{+}\right)$. It is seen in the graph that if $a<a_{c}^{+}$, then $\nu_{n}(a) \approx \frac{3}{4}$, but for $a>a_{c}^{+}$, $H_{n} \simeq$ const so that $\nu_{n}^{\perp}(a) \approx 0$.

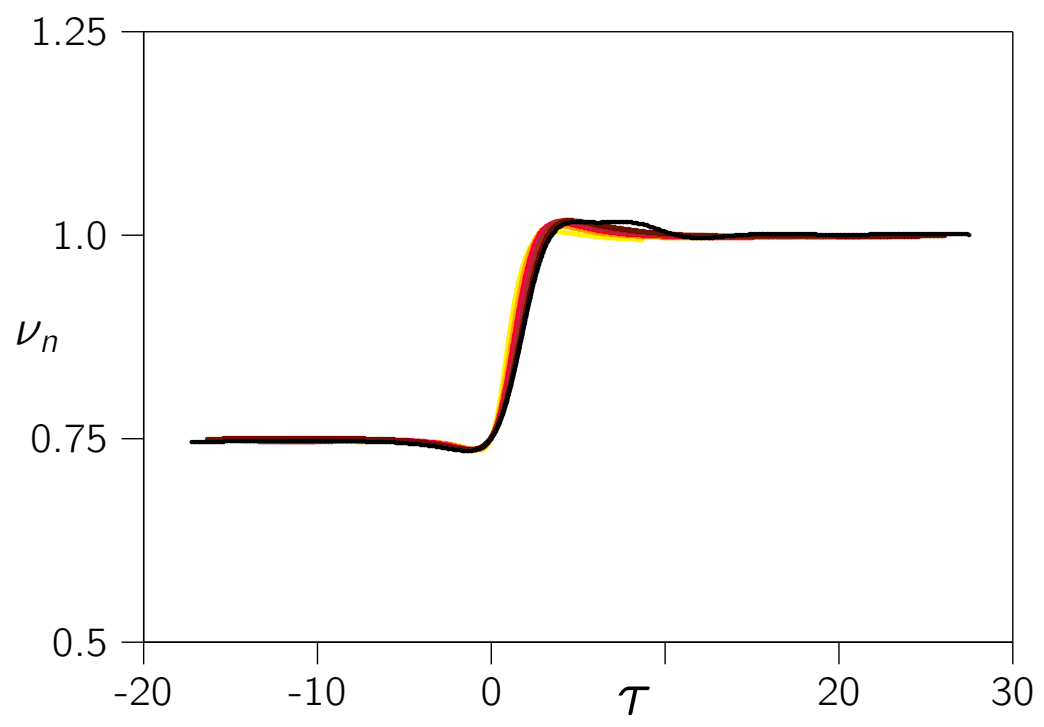

Figure 14: The same data as in figure 13, but now plotted against the rescaled variable $\tau=n^{\phi}\left(a-a_{c}^{+}\right)$, for adsorbing walks in $\mathbb{L}_{2}^{+}$. This rescaling of the activity a collapses the curves to a single underlying curve characterising the metric scaling of the walk in the desorbed and adsorbed phases. The lengths of the walks varied from $n=50$ (yellow) with colours increasing in hue to black when $n=500$ in steps of 50 . 


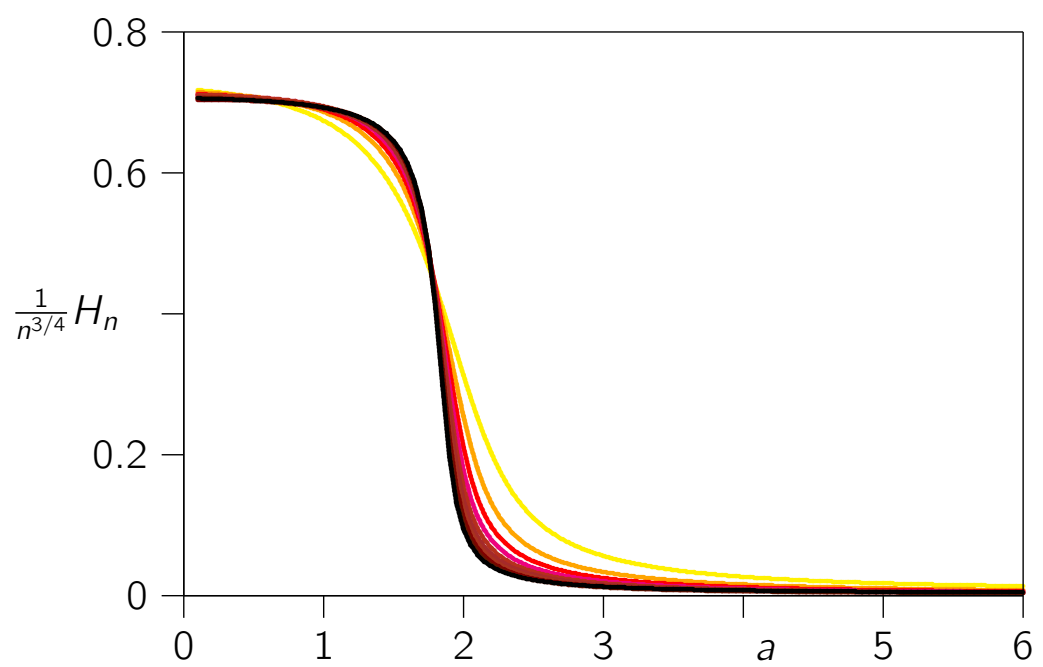

Figure 15: The mean height $H_{n}$ of the endpoint of adsorbing walks in $\mathbb{L}_{2}^{+}$of length $n$ as a function of a. On the left $n^{-\nu} H_{n}$ is plotted as function of a. This quantity decreases sharply as a passes through the adsorption critical point $a_{n}^{+}$. The lengths of the walks varied from $n=50$ (yellow) with colours increasing in hue to black when $n=500$ in steps of 50 .

Since $R_{n}^{2} \sim n^{2 \nu_{a}}$ where $\nu_{a}=\nu=\frac{3}{4}$ if $a<a_{c}^{+}$, and $\nu_{a}=1$ if $a>a_{c}^{+}$, ratios of $R_{n}^{2}$ may be defined by

$$
\frac{R_{n}^{2}}{R_{2 n}^{2}} \approx 2^{-2 \nu_{a}}, \quad \text { or } \quad \frac{2^{2 \nu} R_{n}^{2}}{R_{2 n}^{2}} \approx \begin{cases}1, & \text { if } a<a_{c}^{+} ; \\ 2^{2 \nu-2 \nu_{a}}=2^{-1 / 2}, & \text { if } a>a_{c}^{+} .\end{cases}
$$

In figure 17 the quantity $\frac{2^{2 \nu} R_{n}^{2}}{R_{2 n}^{2}}$ is plotted as a function of the rescaled variable $\tau=$ $n^{1 / 2}\left(a-a_{c}^{+}\right)$for $n$ from 25 to 250 in steps of 25 . In the desorbed phase this ratio should

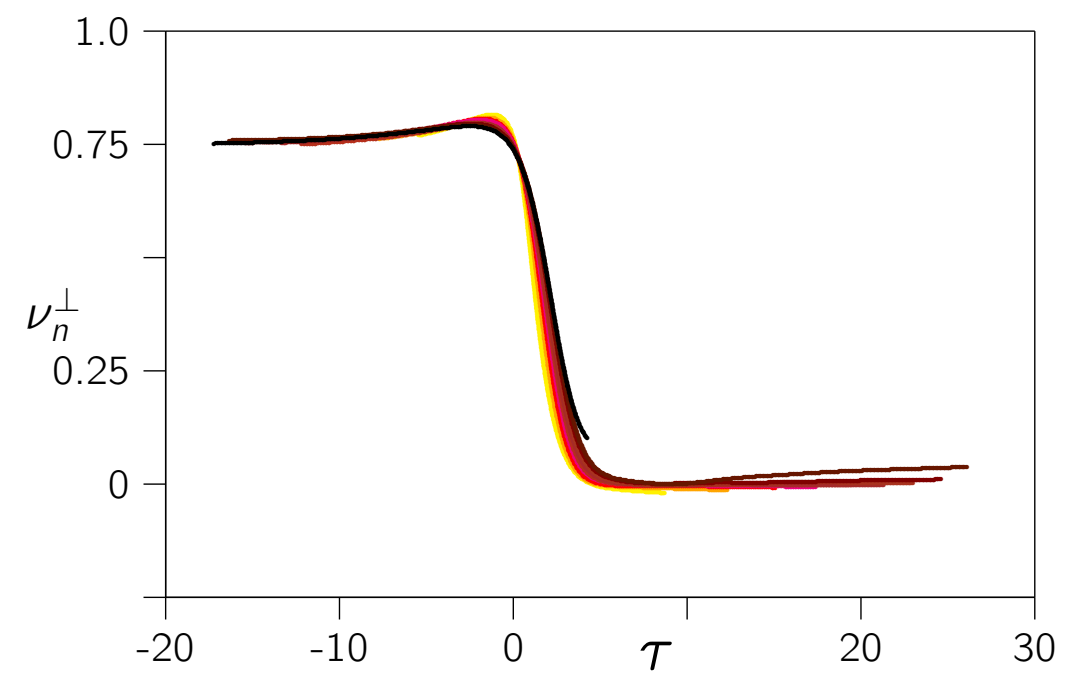

Figure 16: The vertical metric exponent $\nu^{\perp}$ as a function of a for adsorbing walks in $\mathbb{L}_{2}^{+}$. The exponent was estimated from equation (63) by fixing $n$ and then averaging over $m$ for $100 \leq m \leq 500$. With increasing $n$ the exponent changes from $\nu^{\perp}=\nu=\frac{3}{4}$ in the desorbed phase, to $\nu^{\perp}=0$ in the adsorbed phase. Data for $n=500$ and $\tau>5$ did not converge, and is left out of this figure. The lengths of the walks varied from $n=50$ (yellow) with colours increasing in hue to black when $n=500$ in steps of 50 . 


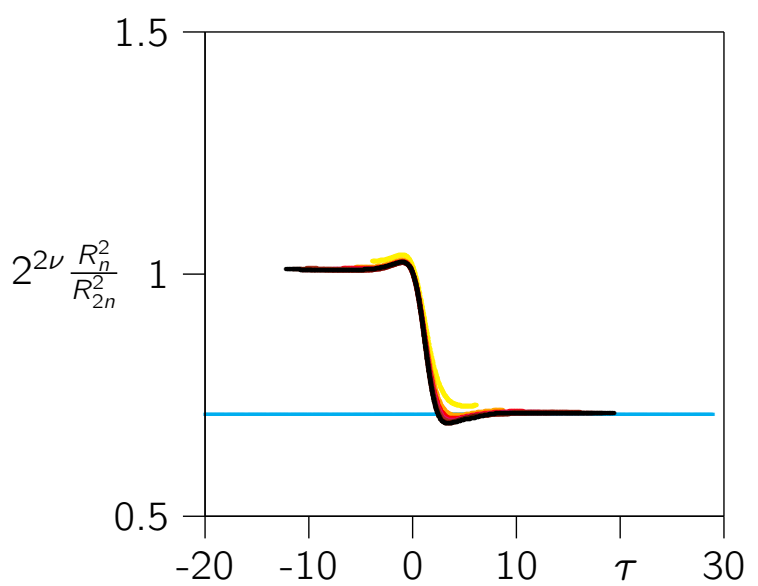

(a)

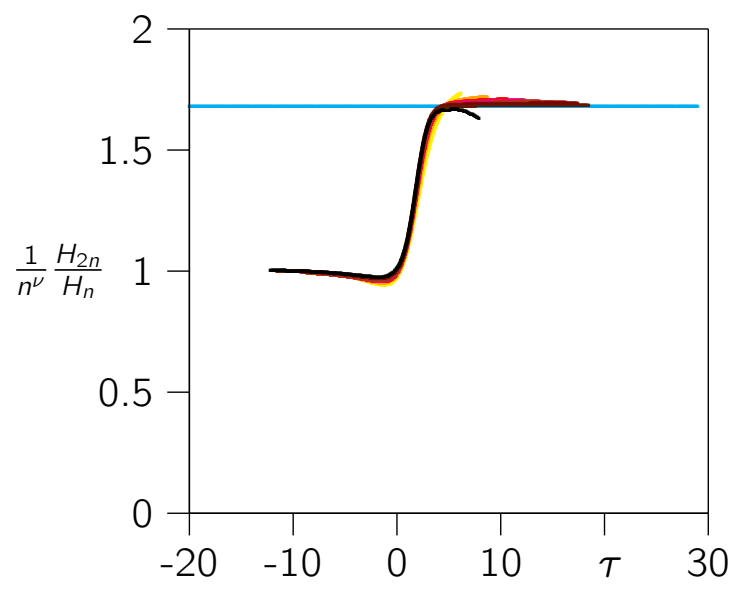

(b)

Figure 17: Metric scaling for adsorbing walks in $\mathbb{L}_{2}^{+}$. (a) The ratio $R_{2 n}^{2} /\left(n^{2 \nu}\right.$ of the mean square radius of gyration plotted as a function of $\tau=n^{\phi}\left(a-a_{c}^{+}\right)$is displayed on the left. The ratio is given in equation (64) and the data shows that it is approximately equal to 1 in the desorbed phase and approximately equal to $2^{3 / 2-2} \approx 0.71$ in the adsorbed phase. (b) The ratio $H_{2 n} /\left(n^{\nu} H_{n}\right)$ plotted as a function of $\tau$. This ratio is defined in equation (65) and is approximately equal to 1 in the desorbed phase, and equal to $2^{3 / 4}$ in the adsorbed phase. The data in both panels are for $n \in\{25,50,75,100, \ldots, 250\}$ and increase in hue from yellow $(n=25)$ to black $(n=250)$.

be equal to 1 , but, in the adsorbed phase, it should be equal to $2^{1.5-2.0} \approx 0.71$. This is clearly seen in the graph. The curves coincide well over the entire range of $n$ and $\tau$, and decreases sharply close to the critical adsorption point at $\tau=0$. A similar approach using the heights of the endpoint involves the ratios

$$
\frac{H_{n}}{H_{2 n}} \approx 2^{-\nu_{a}^{\perp}}, \quad \text { or } \frac{2^{\nu} H_{n}}{H_{2 n}} \approx \begin{cases}1, & \text { if } a<a_{c}^{+} ; \\ 2^{\nu-\nu^{\perp}}=2^{3 / 4}, & \text { if } a>a_{c}^{+} .\end{cases}
$$

The quantity $\frac{2^{\nu} H_{n}}{H_{2 n}}$ is plotted against $\tau$ on the right panel in figure 17 . In the desorbed phase this ratio should be equal to 1 , as seen in the graph. In adsorbed phase the scaling of $H_{n}$ changes, and the ratio should be equal to $2^{3 / 4} \approx 1.68$, as seen in the graph.

\subsection{The generating function}

The generating function is given by the series

$$
G(a, t)=\sum_{n=0}^{\infty} \sum_{v=0}^{n} c_{n}^{+}(v) a^{v} t^{n}
$$

Approximations of $G(a, t)$ is given by the truncated sum

$$
G_{N}(a, t)=\sum_{n=0}^{N} \sum_{v=0}^{n} c_{n}^{+}(v) a^{v} t^{n}
$$

where $c_{n}^{+}(v)$ is approximately enumerated by the GAS algorithm. In this study, $G_{500}(a, t)$ was estimated using the approximate values of $c_{n}^{+}(v)$ obtained in the simulations. 


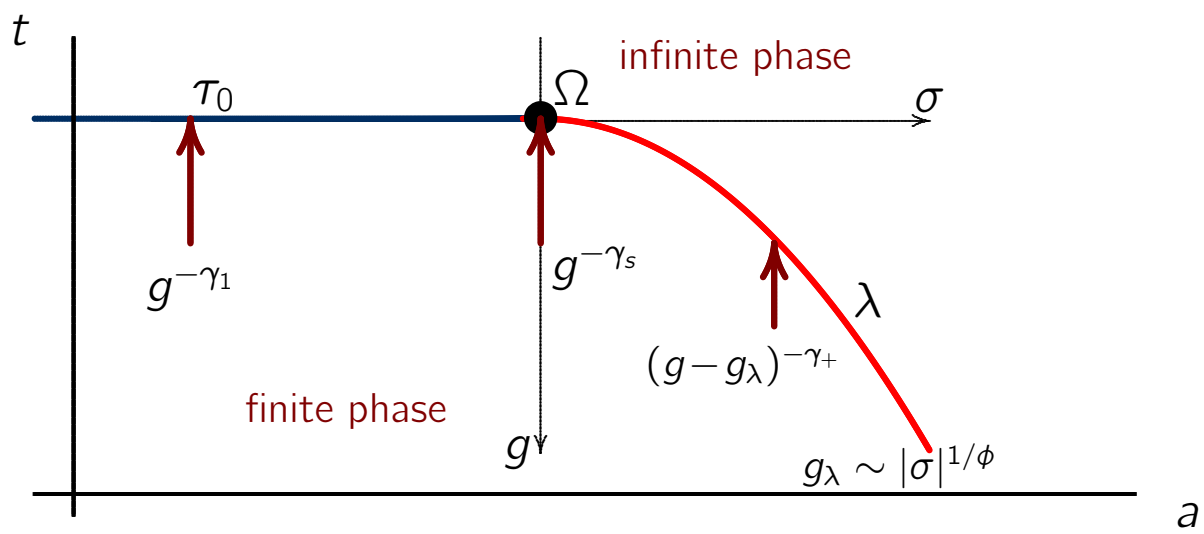

Figure 18: The phase diagram of the generating function $G(a, t)$. The radius of converge of $G(a, t)$ is denoted by $t_{c}^{+}(a)$, and it is the critical curve. This curve is divided into two parts (a transition to a low temperature phase marked by $\tau_{0}$ and a transition to a high temperature phase marked by $\lambda$ ). The $\tau_{0}$ and $\lambda$ curves meet in the critical point $\Omega$ (located at the critical adsorption transition $a_{c}^{+}$). The critical curve separates a "finite phase" (where $G(a, t)$ is convergent and dominated by walks of finite length) from an "infinite phase" (where $G(a, t)$ is divergent). The scaling of $G(a, t)$ is described by setting up a coordinate system $(g, \sigma)$ with origin in $\Omega$, where $\sigma=\left(a-a_{c}^{+}\right)$and $g=\frac{1}{\mu_{2}}-t$. The scaling of $G(a, t)$ on approach to the critical curve is given by equation (69), depending on whether $\sigma<0, \sigma=0$, or $\sigma>0$.

The critical curve $t_{c}^{+}(a)$ of $G(a, t)$ is its radius of convergence as a function of a. By equation (8) and by equation (6),

$$
\log t_{c}^{+}(a)=-\mathcal{F}(a)=-\lim _{n \rightarrow \infty} \frac{1}{n} \log Z_{n}(a) .
$$

The critical curve is shown schematically in figure $18, G(a, t)$ is singular when $t=t_{c}^{+}(a)$, and if $t>t_{c}^{+}(a)$, then $G(a, t)$ is divergent.

The critical curve is parametrized by the scaling fields $(\sigma, g)$ as shown in figure 18 . The critical point when $a=a_{c}^{+}$is located at $\left(a_{c}^{+}, t_{c}^{+}\right)$, where $t_{c}^{+} \equiv t_{c}^{+}\left(a_{c}^{+}\right)$, and it divides the critical curve $t_{c}^{+}(a)$ into two parts. The part marked by $\tau_{0}$ corresponds to a transition to desorbed walks, so that $t_{c}^{+}(a)=1 / \mu_{2}$ in this regime (which is a transition to a high temperature phase). For $a>a_{c}^{+}$the approach to $t_{c}^{+}(a)$ is to adsorbed walks, along the critical curve marked by $\lambda$ (which is a transition to a low temperature phase).

The phase diagram in figure 18 may be described in terms of the coordinates $g=\left(\frac{1}{\mu_{2}}-t\right)$ and $\sigma=\left(a-a_{c}^{+}\right)$. The behaviour of $G(a, t)$ along its singular points along the critical curve is described by

$$
G(a, t) \sim \begin{cases}g^{-\gamma_{1}}, & \text { along } \tau_{0} ; \\ g^{-\gamma_{s}}, & \text { at the critical point } a=a_{c}^{+} ; \\ g^{-\gamma_{+}}, & \text {along } \lambda .\end{cases}
$$

In two dimensions exact values are known for the exponents: The exponent $\gamma_{1}=\frac{61}{64}$ 10] is the entropic exponent of half-space walks, and $\gamma_{s}=\frac{93}{64}[9]$ is the surface exponent of adsorbing half-space walks at the critical point $a_{c}^{+}$. The exponent $\gamma_{+}$is the entropic exponent of adsorbed walks, and is given by the entropic exponent of self-avoiding walks in one dimension lower. In one dimension, this is $\gamma_{+}=1$.

A plot of $G_{N}(a, t)$ is shown in figure 19. The horizontal plane is the $(a, t)$-plane, and the critical curve in figure 18 is shown as a black curve with the critical point shown. 


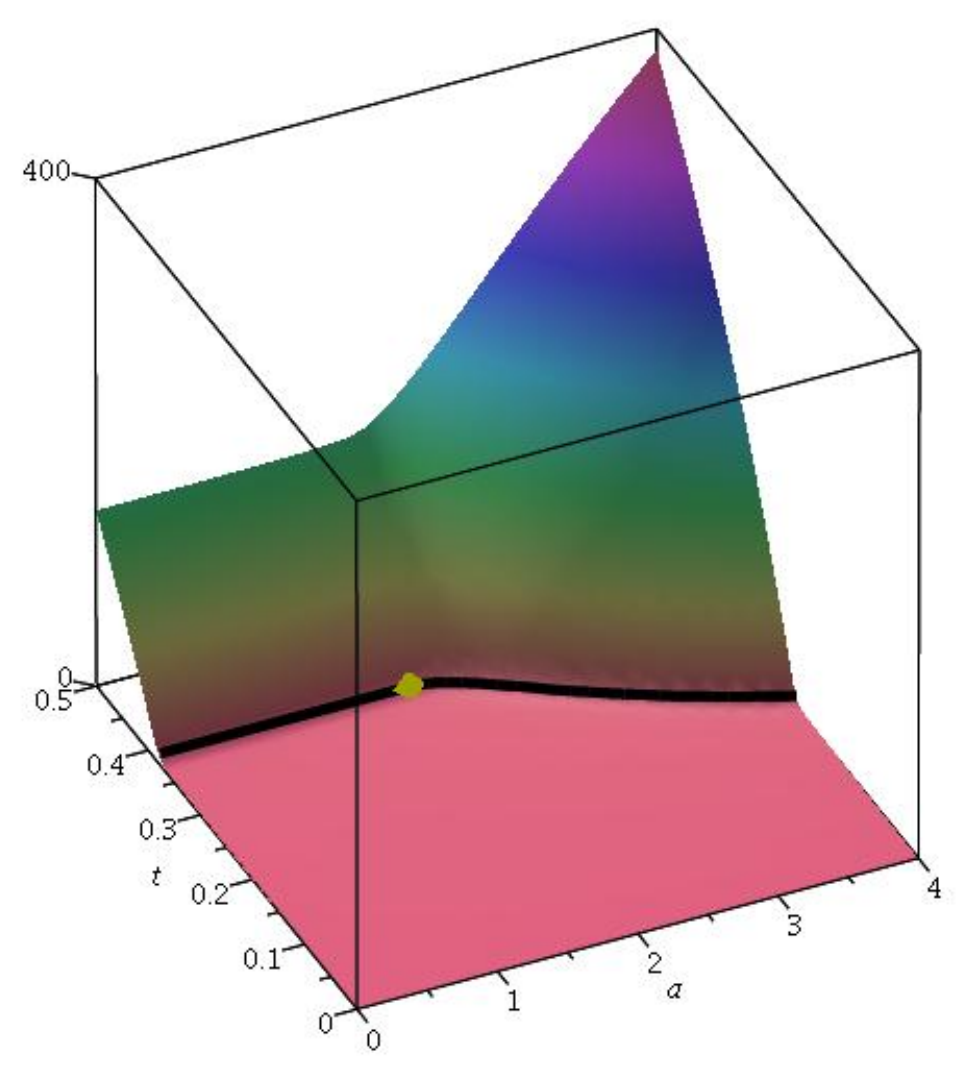

Figure 19: A plot of $\log G_{500}(a, t)$ as a function of $(a, t)$ for adsorbing walks in $\mathbb{L}_{2}^{+}$. The critical curve is the black curve along the surface, and the critical point separating the $\tau_{0}$ and $\lambda$ curves (see figure 18) is denoted. For $t<t_{c}^{+}(a)$ the generating $G(a, t)$ is finite, and for $t>t_{c}^{+}(a)$ it is divergent. These two regimes are clearly visible.

Below the critical curve $G(a, t)$ is finite, and approximated well by $G_{N}(a, t)$ (for large values of $N$ not too close to the critical curve). Above the critical curve $G(a, t)$ is divergent, while $G_{N}(a, t)$, which is a polynomial, is finite.

The exponent $\gamma_{1}$ can be estimated by putting $a=1$ so that $G(1, t) \sim g^{-\gamma_{1}}$ where $g=\left(t_{c}^{+}(1)-t\right)$ and $t_{c}^{+}(1)=\frac{1}{\mu_{2}}$ (and where $\mu_{2}$ is the growth constant of the walks in two dimensions). Thus, estimate $\gamma_{1}$ by noting that

$$
\frac{\log G(1, t)}{\log g}=-\gamma_{1}+\frac{C_{1}}{\log g}+\frac{C_{2}}{\log ^{2} g}+\ldots
$$

Proceed by approximating $G(1, t)$ by $G_{N}(1, t)$ (with $t<t_{c}^{+}(1)$ so that $G_{500}(1, t)$ is a good approximation of $G(1, t)$ ). A least squares fit of the ratio on the left to a quadratic in $\frac{1}{\log g}$ gives the estimate

$$
\gamma_{1}=0.952 \ldots
$$

This result is very close to the exact value $\frac{61}{64}=0.953 \ldots$ Similar analysis for $a>1$ and $a<a_{c}^{+}$gives results slightly larger, since the critical point at $a_{c}^{+}$influences the data in its vicinity for finite values of $N$. 


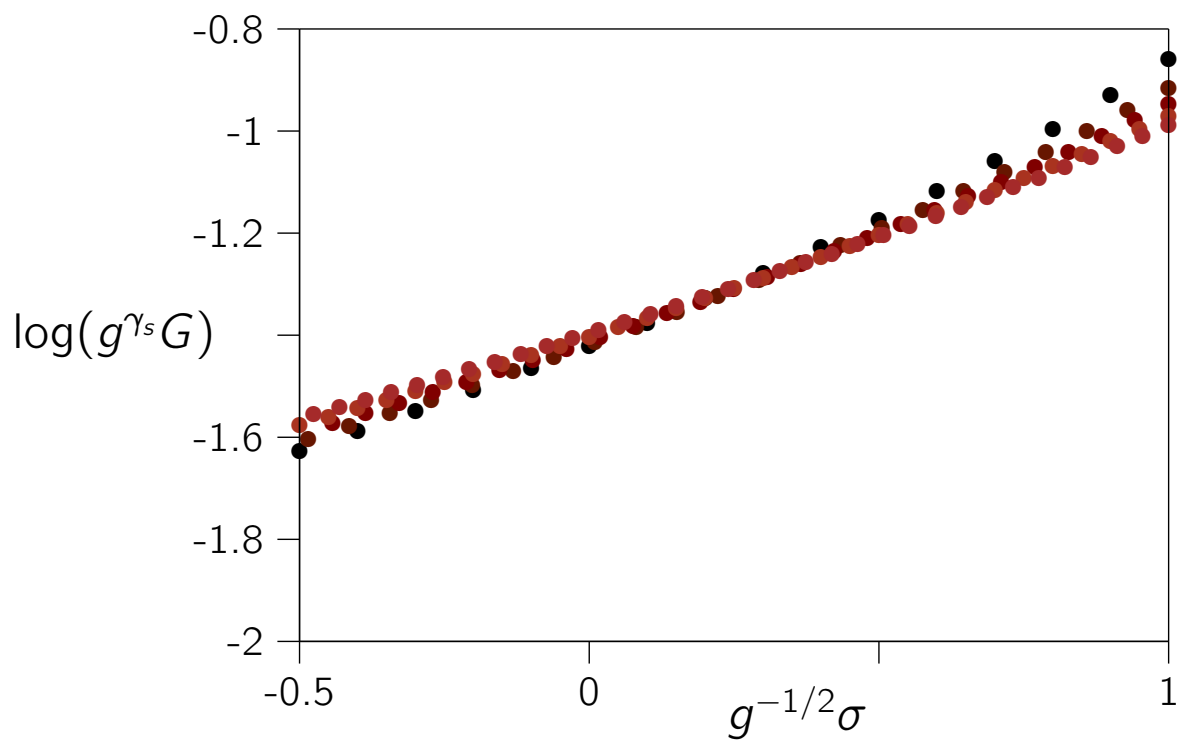

Figure 20: Scaling of the generating function near the critical adsorption point for adsorbing walks in $\mathbb{L}_{2}^{+}$. The data points above are collected for $g \in[0.01,0.05]$ and $-\frac{1}{2} \sqrt{g} \leq \sigma \leq \sqrt{g}$. The surface exponent $\gamma_{s}=\frac{93}{64}$, its exact value.

A similar analysis with $a=a_{c}^{+}$gives the estimate

$$
\gamma_{s}=1.429 \ldots \text {, }
$$

for the surface exponent at the critical adsorption point. This is close in value to the exact result $\frac{93}{64}=1.453 \ldots$

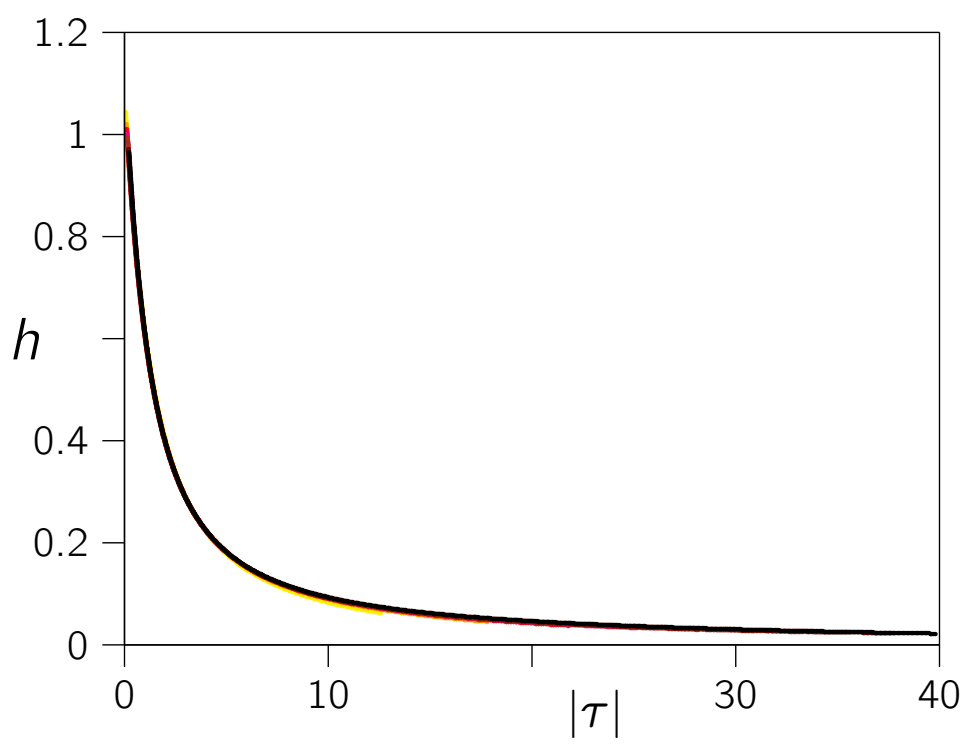

Figure 21: This figure verifies the scaling of the partition function for adsorbing walks in $\mathbb{L}_{2}^{+}$as proposed in equation (77). The curves are plots of $n^{-29 / 64} Z_{n}(a) \mu_{2}^{-n}$ against the combined variable $|\tau|=n^{1 / 2}\left|a-a_{c}^{+}\right|$ (with the critical point approximated by $a_{c}^{+}=1.78$ and $a<a_{c}^{+}$). The curves are walks of lenghts $n \in\{50,100,150, \ldots, 500\}$. All the data collapse to expose the scaling function $h$ in equation (77). The lengths of the walks varied from $n=50$ (yellow) with colours increasing in hue to black when $n=500$ in steps of 50 . 
The case that $a>a_{c}^{+}$may also be analysed. The adsorbed walk should have the statistics of the self-avoiding walk in one dimension, so that the entropic exponent is $\gamma_{+}=1$ (this is the value of the exponent $\gamma$ in one dimension). Putting $a=3.5$ and plotting $Z_{n}^{1 / n}(3.5)$ against $n$ gives an estimate for the critical value $t_{c}^{+}(3.5)$. In this case the data quickly converges to $t_{c}^{+}(3.5)=0.260 \ldots$ (to three decimal places). Assuming that $t_{c}^{+}(3.5)=0.260$ and choosing the scaling field $g=(0.260-t)$ gives the model

$$
\frac{\log G(3.5, t)}{\log g}=-\gamma_{+}+\frac{C_{1}}{\log g}+\frac{C_{2}}{\log ^{2} g}+\ldots
$$

similar to the above, but now with the exponent $\gamma_{+}$. Plotting the left hand side as a function of $\kappa=\frac{1}{\log g}$, and fitting the data to a quadratic in $\kappa$, give the estimate $\gamma_{+}=1.00 \ldots$ There remains, however, some curvature in the model for small values of $g$ (and of $\sigma$ ), so that there may remain strong systematic corrections to this result (however, $\gamma_{+}=1$ is consistent with this result).

In the vicinity of the critical point $\left(a_{c}^{+}, t_{c}^{+}\right)$the generating function should exhibit scaling given by

$$
G(a, t) \sim g^{-\gamma_{s}} f\left(g^{-\phi} \sigma\right),
$$

where $\phi=\frac{1}{2}$ is the crossover exponent and $f$ is a scaling function. That is, plotting $g^{\gamma_{s}} G(a, t)$ against the combined variable $g^{-\phi} \sigma$ should expose the scaling function $f$. In figure 20 this is done by plotting $\log \left(g^{\gamma_{s}} G(a, t)\right)$ against $g^{-1 / 2} \sigma$ for $g=\frac{1}{\mu_{2}}-t \in$ $[0.01,0.05]$ and $\sigma=a-a_{c}^{+} \in\left[-\frac{1}{2} g^{1 / 2}, g^{1 / 2}\right]$.

The partition function (see equation (6)) should also exhibit scaling for large $n$, given by

$$
Z_{n}(a) \sim n^{\gamma_{t}-1} h\left(n^{\phi}\left(a_{c}^{+}-a\right)\right) \mu_{a}^{n}
$$

where $\log \mu_{a}=\mathcal{F}(a)$ and $\log \mu_{a}=-\log t_{c}^{+}(a)$. The exponent $\gamma_{t}$ can be related to the $\gamma$-exponents as $a \rightarrow a_{c}^{+}$(that is, as $\sigma \rightarrow 0$ ), and namely to the surface exponent $\gamma_{s}$, where the walk is critical with respect to the adsorption transition. By noting that $G(a, t)=\sum_{n} Z_{n}(a) t^{n}$, and approximating the summation by an integral, it follows that $G(a, t) \sim g^{-\gamma_{t}} h(0)$ if $a=a_{c}^{+}$and $g=t_{c}^{+}-t$. This shows that

$$
\gamma_{t}-1=\gamma_{s}-1=\frac{93}{64}-1=\frac{29}{64} \text {. }
$$

That is, when $a$ is close to $a_{c}^{+}$(and $a<a_{c}^{+}$), the partition function has asymptotic behaviour

$$
Z_{n}(a) \sim n^{29 / 64} h\left(n^{\phi}\left(a_{c}^{+}-a\right)\right) \mu_{a}^{n} .
$$

This result may be tested by plotting $n^{-29 / 64} Z_{n}(a)\left(t_{c}^{+}(a)\right)^{n}$ against $|\tau|=n^{1 / 2}\left|a-a_{c}^{+}\right|$. All the data should collapse to the same universal curve exposing the scaling function $h$. This is done with $a<a_{c}^{+}$(and $\left.t_{c}^{+}(a)=\frac{1}{\mu_{2}}\right)$ in figure 21 for $n \in\{50,100,150, \ldots, 500\}$ and $0 \leq a<a_{c}^{+}$.

\subsection{Adsorbing walks in the cubic lattice}

The (finite size) free energy $\mathcal{F}_{n}(a)$ is a function of the combined variable $\tau=n^{1 / 2}\left(a-a_{c}^{+}\right)$ (see equations (10) and (12); note that $\phi=\frac{1}{2}$ for adsorbing walks in three dimensions [19]). Plotting $\mathcal{F}_{n}(a)$ against $\tau$ for data in the cubic lattice gives a graph similar to figure 


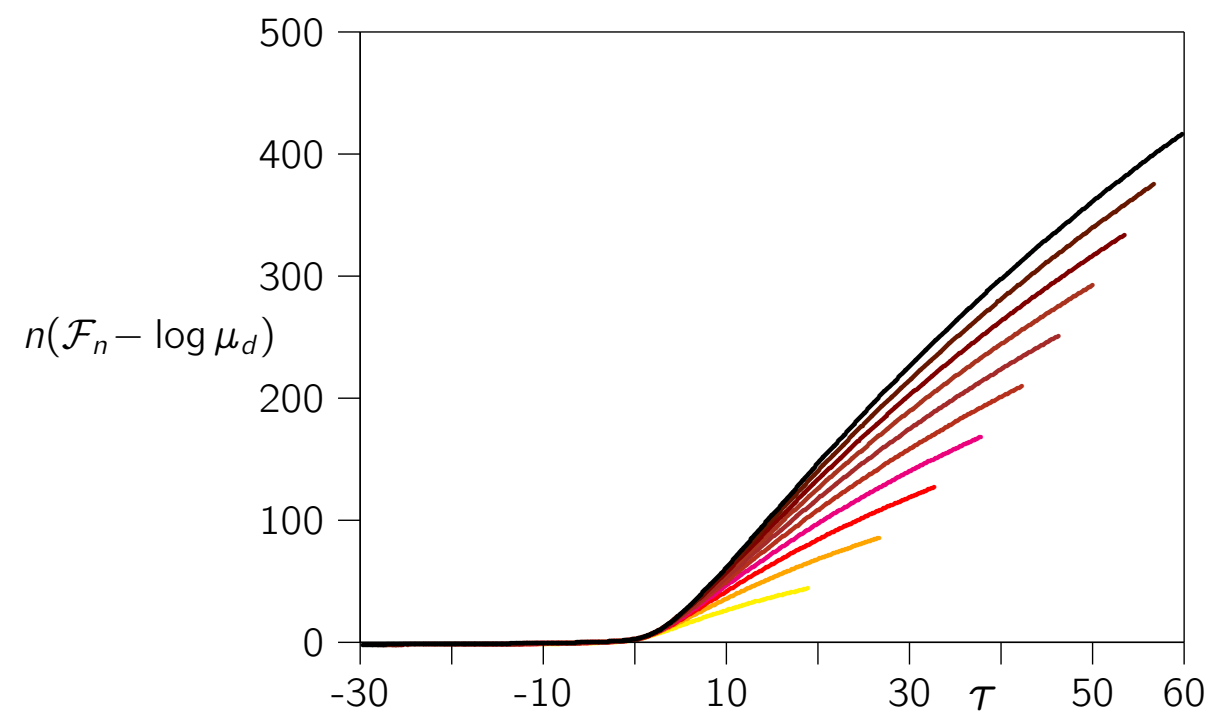

Figure 22: The scaled free energy $n\left(\mathcal{F}_{n}(a)-\log \mu_{d}\right)$ as a function of $\tau=n^{1 / 2}\left(a-a_{c}^{+}\right)$for adsorbing walks in $\mathbb{L}_{3}^{+}$. The lengths of the walks varied from $n=50$ (yellow) with colours increasing in hue to black when $n=500$ in steps of 50. The data collapse for small $|\tau|$ (this is the critical scaling regime), and will approach a limiting curve as $n \rightarrow \infty$, also for large $\tau$, given by the limiting free energy.

6. In figure 22 the scaled free energy $n\left(\mathcal{F}_{n}(a)-\log \mu_{3}\right)$ is plotted against $\tau$. The data shows a clear transition in the model from a desorbed to an adsorbed phase.

Derivatives of the free energy to log a gives the (finite size) energy density $\mathcal{E}_{n}(a)$ and (finite size) specific heat $\mathcal{C}_{n}(a)$. These are plotted in figure 23 against log a and in figure 24 against $\tau$. In these plots, as in figure 22, the critical point was approximated by $a_{c}^{+}=1.31$. This is a close approximation of the best estimate for the critical point from our data (see equation (79)).
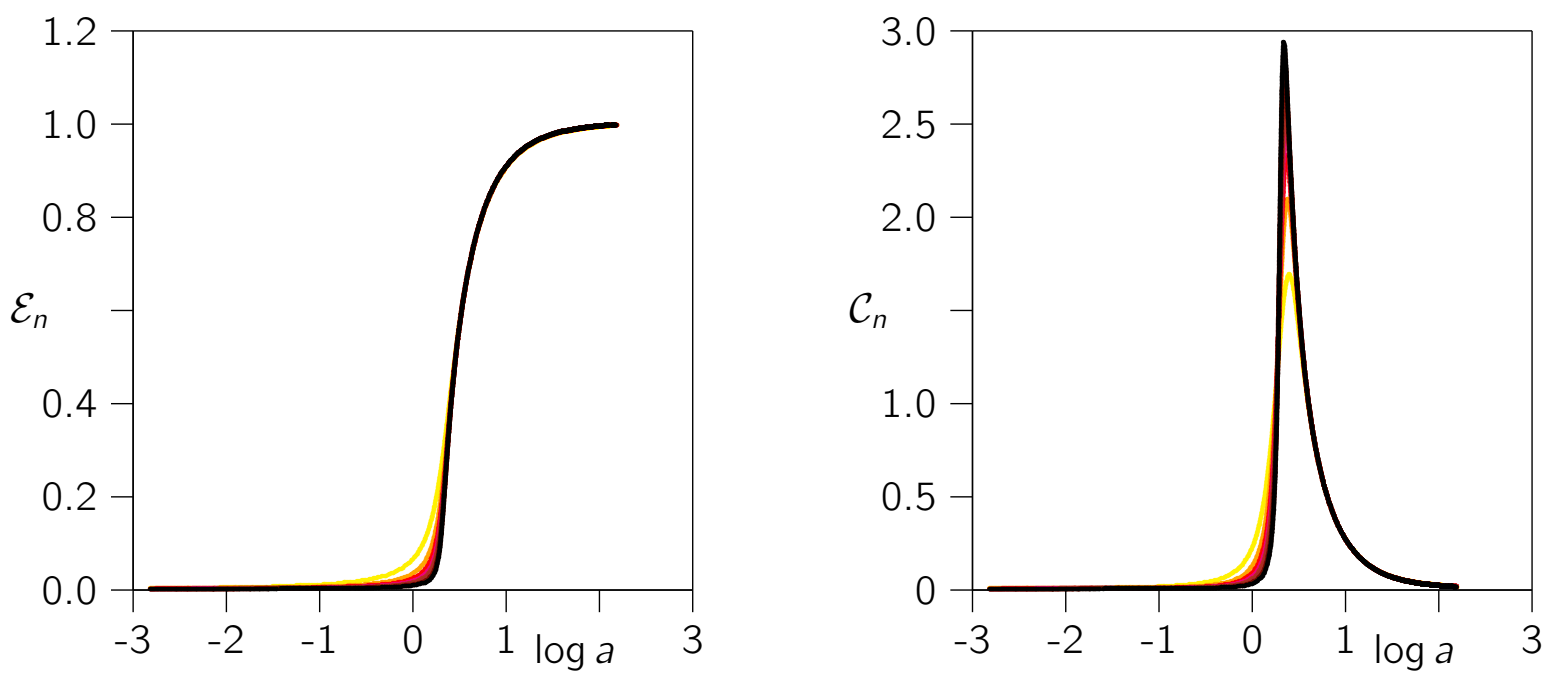

Figure 23: The energy $\mathcal{E}_{n}(a)$ and specific heat $\mathcal{C}_{n}($ a) (see equation $(13)$ ) as a function of log a for adsorbing walks in the cubic lattice. See figure 7 for the similar results in the square lattice. The lengths of the walks varied from $n=50$ (yellow) with colours increasing in hue to black when $n=500$ in steps of 50 . 

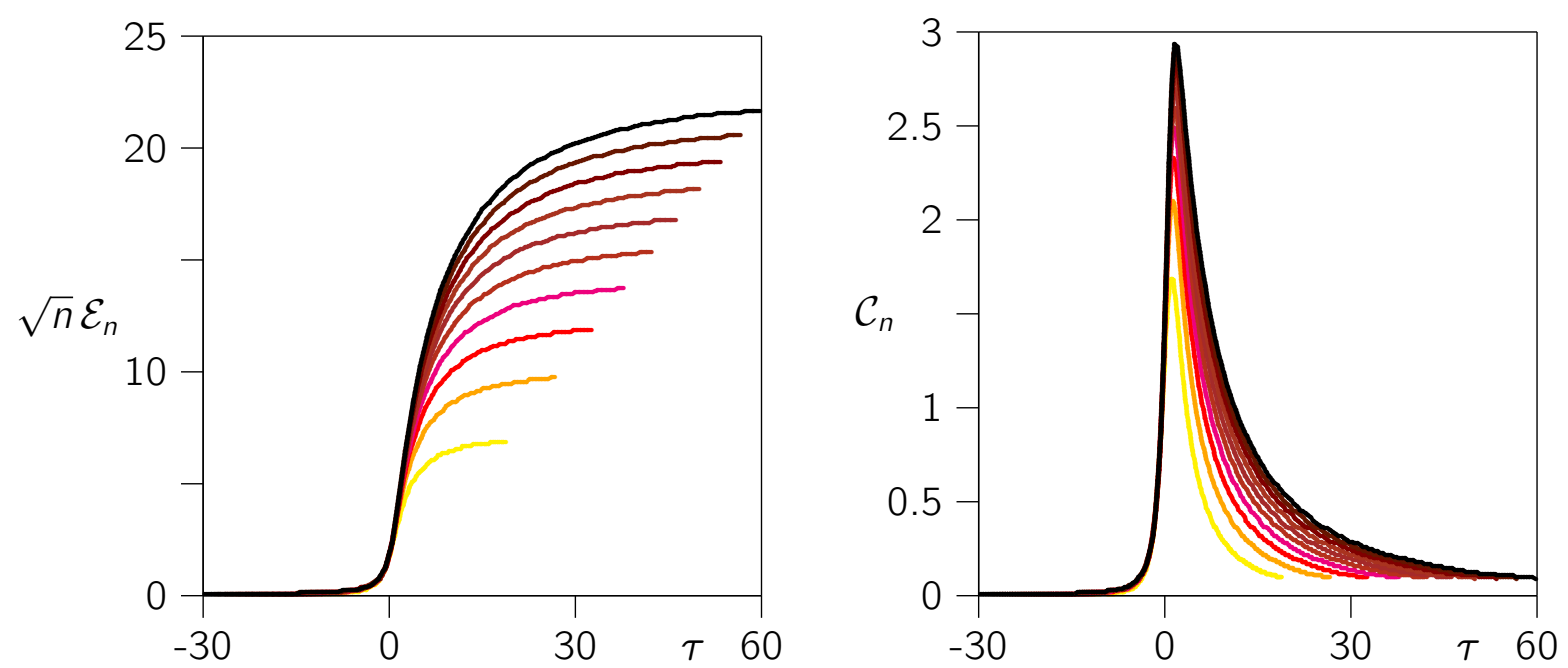

Figure 24: The scaled energy density $\sqrt{n} \mathcal{E}_{n}(a)$ (left) and the specific heat $\mathcal{C}_{n}(a)$ (right) for adsorbing walks in $\mathbb{L}_{3}^{+}$as a function of the $\tau=n^{1 / 2}\left(a-a_{c}^{+}\right)$for $n$ in steps of 50 to 500 . The lengths of the walks varied from $n=50$ (yellow) with colours increasing in hue to black when $n=500$ in steps of 50 . The data collapse for small values of $|\tau|$ (this is the critical scaling regime which contains the critical point). For larger values of $|\tau|$ the curves will approach a limiting curve as $n \rightarrow \infty$ given by the limiting free energy.

3.3.1. Location of the critical point $a_{c}^{+}$: The location of the critical adsorption point can be determined using the same analysis as in section 3.1.1, and in particular using equation (50) as a starting point. That, is for given values of $n$ and $m$, an estimate $a_{n, m}^{+}$ of $a_{c}^{+}$can be obtained by solving for $a$ in

$$
\frac{\log \left(n \mathcal{E}_{n}(a)\right)}{\log \left(m \mathcal{E}_{m}(a)\right)} \frac{\log m}{\log n}=1 .
$$

Here, the choice $m=n-100$ worked well, and $n$ was assigned values starting at $n=200$ to $n=500$ in steps of 1 . The estimates $a_{n}^{+} \equiv a_{n, n-100}^{+}$showed a dependence on $n$, systematically decreasing with increasing $n$. The best estimate is obtained by using the model $a_{n}^{+}=a_{c}^{+}-\frac{c_{1}}{\log ^{2} n}$ suggested by equation (51). A least squares fit for all $n \in[200,500]$ gives the best estimate

$$
a_{c}^{+}=1.3055 \pm 0.0061 .
$$

The confidence interval is obtained by doubling the square root of the variance of the estimates $a_{n}^{+}$. This result is slightly smaller than the result in reference [23], namely $a_{c}^{+}=1.334 \pm 0.027$ (obtained by using a Multiple Markov Chain implementation of the Berretti-Sokal algorithm [6]).

The estimate (79) can be used to determine the crossover exponent $\phi$. This is again done by considering the scaling of the specific heat (equation (13)). It is expected that $\mathcal{C}_{n}\left(a_{c}^{+}\right) \sim n^{\alpha \phi} h_{c}(0)$ when $a=a_{c}^{+}$. An estimate of $\alpha \phi$ is obtained by computing $\frac{\log \mathcal{C}_{n}\left(a_{c}^{+}\right)}{\log n}$ for a range of values of $n$ (in this case $100 \leq n \leq 500$ ). The average is taken as the best estimate and a confidence interval is estimated by doubling the square root of the variance of the estimates. This gives $\alpha \phi=0.0106 \pm 0.0116$. Determine the best estimate for $\phi$ by using equation (11):

$$
\phi=0.5053 \pm 0.0053 \text {. }
$$




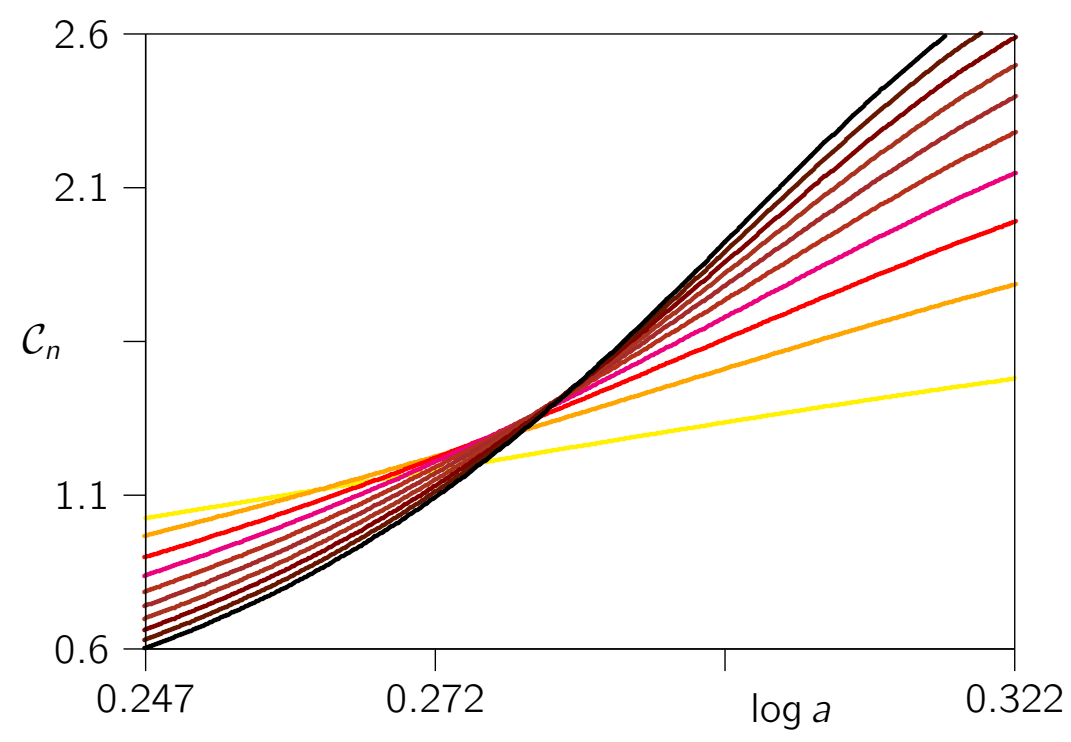

Figure 25: The specific heat curves in figure 23 for adsorbing walks in $\mathbb{L}_{3}^{+}$intersect each other close to the critical adsorption point. This figure is a magnification of the curves close to where they intersect. See figure 9 for the similar results in the square lattice. The lengths of the walks varied from $n=50$ (yellow) with colours increasing in hue to black when $n=500$ in steps of 50 .

This result compares well with the estimate $\phi=0.5005 \pm 0.0036$ for adsorbing walks in reference [23].

3.3.2. The critical point $a_{c}^{+}$and the specific heat $\mathcal{C}_{n}(a)$ : The best estimate for $a_{c}^{+}$above (see equation (790) should be examined by comparing it to estimates obtained from the specific heat curves in figure 23. These curves intersect each other near $a_{c}^{+}$, and the region containing the intersections is magnified in figure 25.

The locations of the intersections between the specific heat curves in figure 25] is a function of $n$ and are estimates of the critical adsorption point $a_{c}^{+}$. By plotting the intersections between $\mathcal{C}_{n}(a)$ and $\mathcal{C}_{n+100}(a)$ against $\frac{1}{\sqrt{n}}$ (see figure 26. where $n=2 \mathrm{~N}$ and $N \in[23,200])$; it is seen that the intersections fall approximately along a curve, which may be extrapolated to its intersection with the vertical axis. This gives a rough estimate of the critical point $\log a_{c}^{+}$as being located in the the interval $[1.33,1.35]$. A more accurate extrapolation is done by using a linear least squares model to extrapolate to $n=\infty$. Fitting to the model $a_{c}^{+}+\frac{a_{0}}{\sqrt{n}}+\frac{a_{1}}{n}$, for all $n \geq 50$, gives the estimate $\log a_{c}^{+} \approx 1.337$. By examining the spread of the data in figure 26, a confidence interval can be estimated:

$$
\log a_{c}^{+}=1.337 \pm 0.020 .
$$

This estimate is slightly larger than the estimate in equation $(79)$, but is consistent with the estimate $a_{c}^{+}=1.334$ in reference [23]. However, the noise in the data in figure 26 makes this a less reliable estimate.

Equation (56) is equally valid for adsorbing walks in the cubic lattice. The last term on the right hand side is equal to zero when $a=a_{c}^{+}$. Thus, by plotting $\log \left(\mathcal{C}_{n}(a) / \mathcal{C}_{m}(a)\right)$ against $\log (n / m)$, a set of curves should be seen which intersect when $a=a_{c}^{+}$. At this 


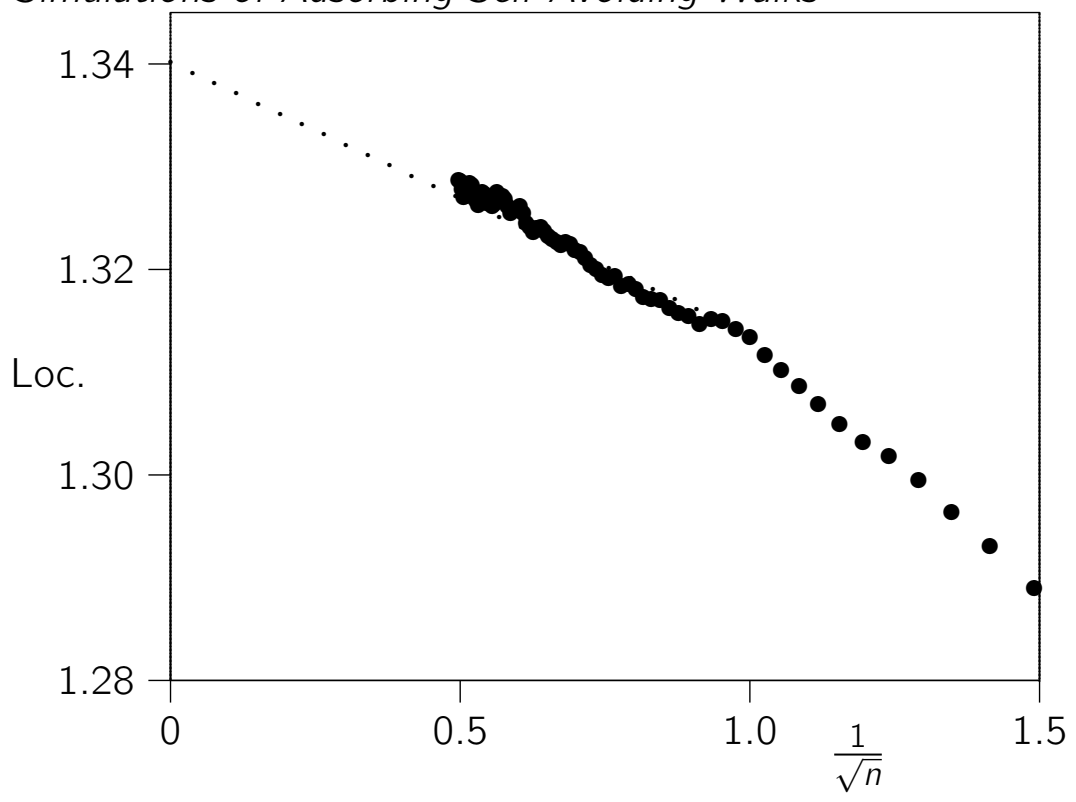

Figure 26: Extrapolating the intersections between specific heat curves for adsorbing walks in $\mathbb{L}_{3}^{+}$. These data points are the locations of intersections between specific heat curves for $n$ and for $n+100$ (and for even $n$ and $n \in[46,400]$ ). The points (except for some points at the largest values of $n$ ) approximately line up linearly if plotted against $1 / \sqrt{n}$. The location of the critical point can be estimated by extrapolating the line to the left vertical axis; this gives the estimate in equation (81).

point the coefficient of $\log \frac{n}{m}$ is an estimate of $\alpha \phi$. Since $\alpha=0$ in this model (and $\left.\phi=\frac{1}{2}\right)$, the critical point can also be determined by solving for $a$ in

$$
\log \left(\frac{\mathcal{C}_{n}(a)}{\mathcal{C}_{m}(a)}\right)=0 .
$$

Solving this for $n \in[150,500]$ and $m \in[n-100, n-10]$ gives a large collection of estimates. The average is

$$
a_{c}^{+}=1.324 \pm 0.012 \text {, }
$$

where the confidence interval is one-half of the largest difference between two estimates in the collection. This result is smaller than the estimate in equation (81), and larger than the best estimate in equation (79).

These results indicate that there may remain sources of systematic errors in the data and in the analysis, and that the estimates for $a_{c}^{+}$should be considered in this context.

3.3.3. The microcanonical density function: The microcanonical density function of visits in adsorbing positive walks is determined from the microcanonical data in the model, and is given by

$$
P^{+}(\epsilon)=\lim _{n \rightarrow \infty}\left(c_{n}^{+}(\lfloor\epsilon n\rfloor)\right)^{1 / n}=\lim _{n \rightarrow \infty} P_{n}^{+}(\epsilon),
$$

where $P_{n}^{+}(\epsilon)=\left(c_{n}^{+}(\lfloor\epsilon n\rfloor)\right)^{1 / n}$ is a finite size approximation to $P^{+}(a)$. Existence of $P^{+}(\epsilon)$ can be shown (see for example reference [22]), and $\log P^{+}(\epsilon)$ is a concave function of $\epsilon$.

$P^{+}(\epsilon)$ can be determined by interpolating the finite size approximations $P_{n}^{+}(\epsilon)$ and then extrapolating to $n=\infty$ by fitting a least squares model to the data. In figure 27 


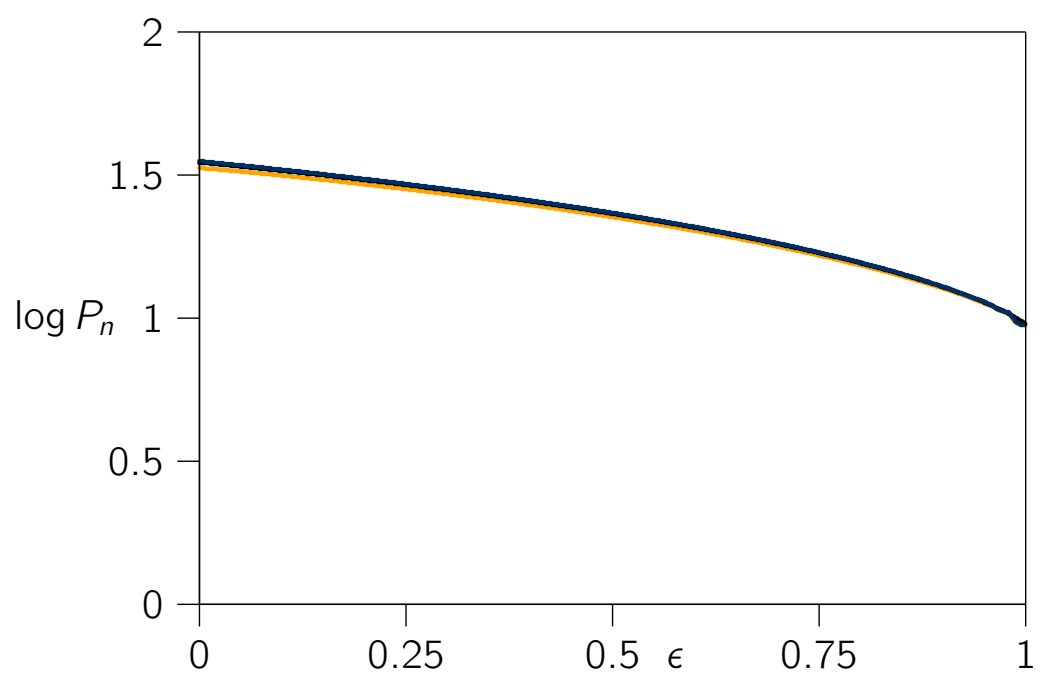

Figure 27: The microcanonical density function $P^{+}(\epsilon)$ of visits for adsorbing walks in $\mathbb{L}_{3}^{+}$. These data the finite size approximations $P_{n}^{+}(\epsilon)$ to $P^{+}(\epsilon)$ for $n=100$ and $n=500$, as well as the extrapolated estimate of $P^{+}(\epsilon)$. The right derivative of $\log P^{+}(\epsilon)$ at $\epsilon=0$ is an estimate of the location of the critical adsorption point $a_{c}^{+}$in the model.

the data for the extrapolated function $P^{+}(\epsilon)$ is plotted together with $P_{n}^{+}(\epsilon)$ for $n=100$ and $n=500$.

A least squares fit of a quadratic to $\log P^{+}(\epsilon)$ for $\epsilon \in[0,0.1]$ gives $\log P^{+}(\epsilon) \approx$ $1.54378-0.28704 \epsilon-0.08784 \epsilon^{2}$. By taking the right derivative and then taking $\epsilon \rightarrow 0^{+}$, an estimate for the critical point is obtained:

$$
a_{c}^{+} \approx 1.332 \text {. }
$$

The free energy is the Legendre transform of $\log P^{+}(\epsilon)$. This may be estimated by fitting a polynomial to $\log P^{+}(\epsilon)$. If a cubic polynomial in $\epsilon$ is fitted to $\log P^{+}(\epsilon)$ for $0 \leq \epsilon \leq 0.5$, then the estimated free energy for $a>a_{c}^{+}$is approximately

$$
F(a) \approx 1.6526-0.4240 \log a-(0.3184-1.319 \log a) \sqrt{-0.2438+1.0105 \log a} \text {. }
$$

The critical point can be estimated as that location where the square root in the above is zero. This gives $a_{c}^{+} \approx 1.273$. Similarly, the factor $(0.3184-1.319 \log a)$ vanishes when $a_{c}^{+} \approx 1.273$. These estimates are far less secure than the estimates above, and are also smaller.

3.3.4. Metric data: The mean square radius of gyration $R_{n}^{2}$ and mean height $H_{n}$ of the endpoint of the walk can be calculated as a function of $a$. In the desorbed phase (for $a<a_{c}^{+}$) it is expected that $R_{n}^{2} \sim n^{2 \nu}$, and $H_{n} \sim n^{\nu}$, where $\nu=0.587 \ldots[11$ is the metric exponent. This scaling changes in the adsorbed phase (when $a>a_{c}^{+}$); in this phase it should be the case that $R_{n}^{2} \sim n^{3 / 2}$ and $H_{n} \sim$ constant, since an adsorbed walk in the cubic lattice should have the statistics of a walk in one dimension lower.

These expectations are supported by the data, as seen, for example, in figure 28 , where data for the mean square radius of gyration are plotted as a function of $a$. These graphs clearly show two scaling regimes, namely a high temperature phase (when $a<a_{c}^{+}$) where the walk has bulk critical exponents and is desorbed, and a low temperature phase 

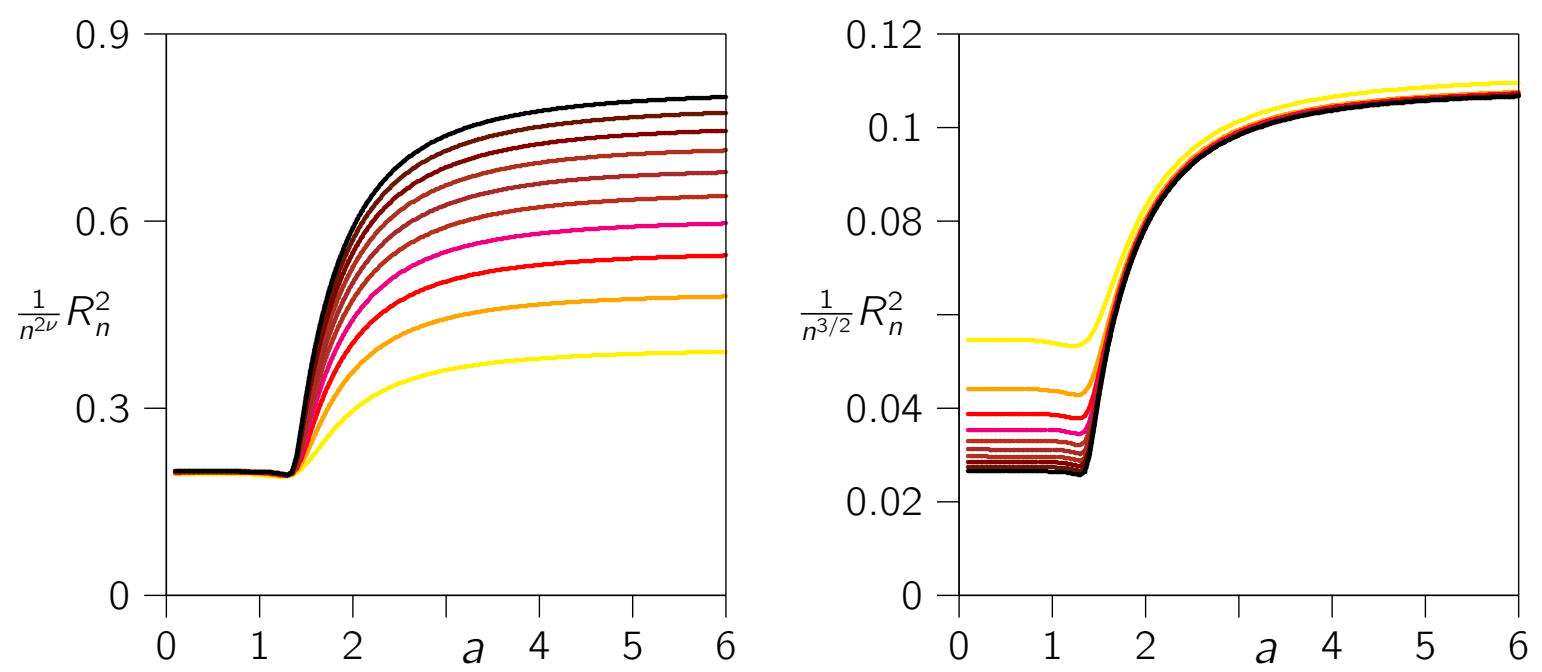

Figure 28: The mean square radius of gyration as a function of a for adsorbing walks in $\mathbb{L}_{3}^{+}$. In the left panel $R_{n}^{2}$ is divided by $n^{2 \nu}$. For $a<a_{c}^{+}$this shows that $R_{n}^{2} \sim n^{2 \nu}$, and for $a>a_{c}^{+} R_{n}^{2}$ increases in size faster than $n^{2 \nu}$ (since it is adsorbed, it stays in the vicinity of the adsorbing boundary, and it should be the case that $R_{n}^{2} \sim n^{3 / 2}$ in this regime. This is seen in the right panel, where $R_{n}^{2}$ is divided by $n^{3 / 2}$. For $a>a_{c}^{+}$the curves collapse to a single underlying, exposing the scaling in the adsorbed phase. The lengths of the walks varied from $n=50$ (yellow) with colours increasing in hue to black when $n=500$ in steps of 50 .

where the walk stays near the adsorbing boundary and has critical exponents of a walk in one dimension lower.

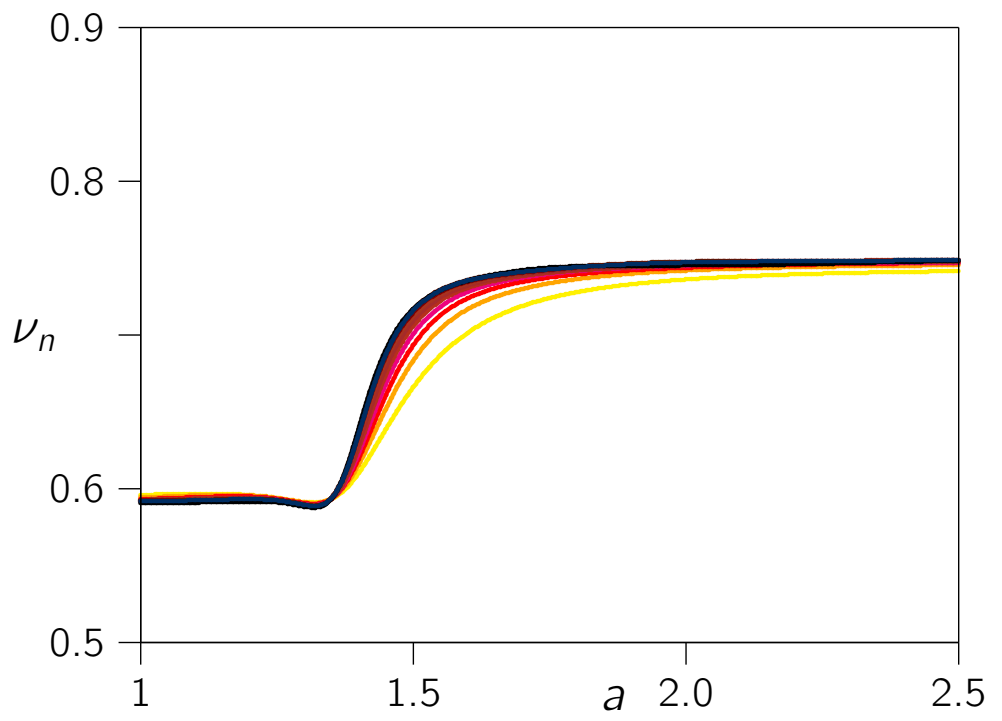

Figure 29: The metric exponent as a function of a for adsorbing walks in $\mathbb{L}_{3}^{+}$. The exponent was estimated from equation (87) by fixing $n$ and then averaging over $m$ for $50 \leq m \leq 500$. With increasing $n$ the change from $\nu=0.588 \ldots$ in the desorbed phase, to $\nu=0.75$ in the adsorbed phase will become a step function at $a=a_{c}^{+}$. The lengths of the walks varied from $n=50$ (yellow) with colours increasing in hue to black when $n=500$ in steps of 50 . 


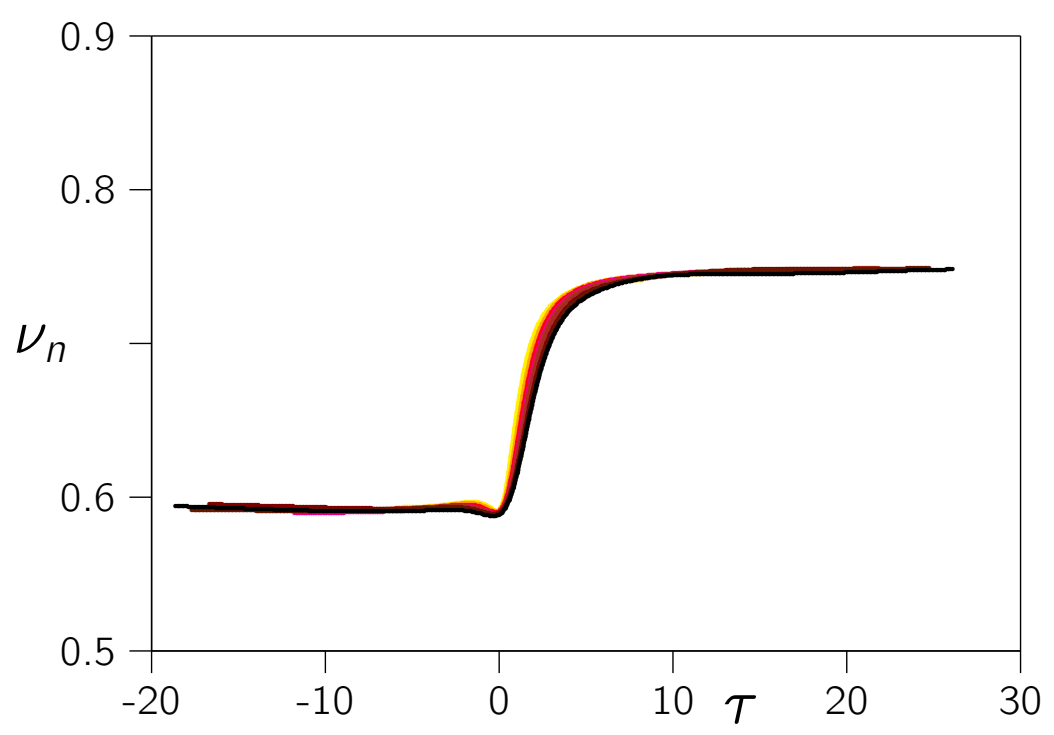

Figure 30: The metric exponent as a function of the rescaled variable $\tau=n^{\phi}\left(a-a_{c}^{+}\right)$for adsorbing walks in $\mathbb{L}_{3}^{+}$. The lengths of the walks varied from $n=50$ (yellow) with colours increasing in hue to black when $n=500$ in steps of 50 .

The metric exponent $\nu$ may be estimated from $R_{n}^{2}$ by examining the ratios

$$
2 \nu_{n, m}(a)=\frac{\log \left(R_{n}^{2} / R_{m}^{2}\right)}{\log (n / m)} .
$$

Here, $\nu_{n, m}(a)$ is a function of $n$ and $m$. By averaging over $m$, the estimate $\nu_{n}(a)=$ $\left\langle\nu_{n, m}\right\rangle_{m}$ can be determined. Taking the average for $100 \leq m \leq 500$ in multiples of 5 (and for $m \neq n$ ) gives an estimate for $\nu_{n}(a)$. The results are plotted in figure 29 for $n \in\{50,100,150, \ldots, 500\}$. The data for $a \leq 1.2$ give $\nu \approx 0.592$, and for $a \geq 1.7$, $\nu \approx 0.740$.

The scaling of $\nu_{n}(a)$ as a function of $\tau=n^{\phi}\left(a-a_{c}^{+}\right)$can be uncovered by plotting the data in figure 29. This gives a set of curves which are very close to one another, uncovering a scaling function $\nu$ where $\nu_{n}(a)=\nu(\tau)$.

The average height of the endpoint of the walk is plotted as a function of $a$ in figure 31. The left panel displays the height normalised by division with $n^{\nu}$ and gives a set of curves which increase with $n$ for $a<a_{c}^{+}$, and decrease with $n$ for $a>a_{c}^{+}$. The curves intersect close to the critical adsorption point, and the limiting curve (in the $n \rightarrow \infty$ limit) should be a step function with critical point at $a=a_{c}^{+}$.

The vertical metric exponent $\nu^{\perp}$ can be estimated from $H_{n}$, by using a method similar to equation (87), namely an approximation by examing the ratios of $H_{n}$ :

$$
\nu_{n, m}^{\perp}(a)=\frac{\log \left(H_{n} / H_{m}\right)}{\log (n / m)} .
$$

The exponent is approximated by $\nu_{n}^{\perp}(a)=\left\langle\nu_{n, m}(a)\right\rangle_{n}$. Taking the average for $100 \leq$ $m \leq 500$ for fixed $n$ gives esimates for $\nu_{n}^{\perp}(a)$. If $a<a_{c}^{+}$, then $\nu_{n}^{\perp}(a)$ should have value approximately equal to $\nu$; that is, $\nu_{n}(a) \approx 0.58 \ldots$, and for $a>a_{c}^{+}, H_{n} \simeq$ const so that $\nu_{n}^{\perp}(a) \approx 0$ in this phase. The results are plotted in figure 32 against $\tau=n^{\phi}\left(a-a_{c}^{+}\right)$. 


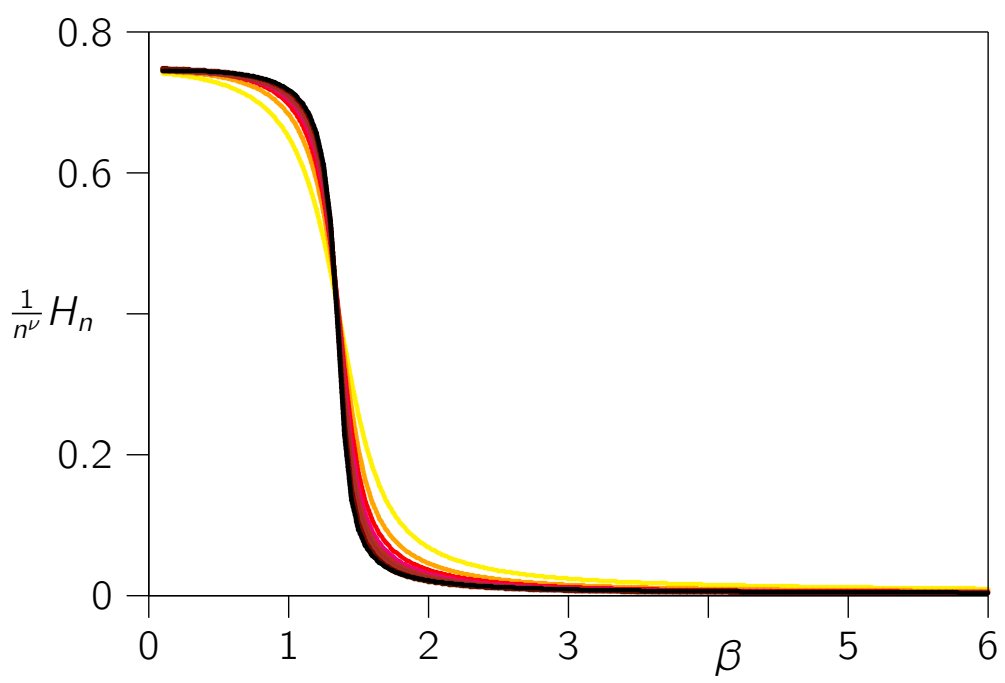

Figure 31: The mean height $H_{n}$ of the endpoint of adsorbing walks of length $n$ as a function of $a$ for adsorbing walks in $\mathbb{L}_{3}^{+}$. On the left $n^{-\nu} H_{n}$ is plotted as function of a. This quantity decreases sharply as a passes through the adsorption critical point $a_{n}^{+}$. This behaviour is also seen on the right, where $n^{-1} H_{n}$ is plotted as a function $n$. The lengths of the walks varied from $n=50$ (yellow) with colours increasing in hue to black when $n=500$ in steps of 50 .

Since $R_{n}^{2} \sim n^{2 \nu_{a}}$ where $\nu_{a}=\nu \approx 0.588 \ldots$ if $a<a_{c}^{+}$, and $\nu_{a}=\frac{3}{4}$ if $a>a_{c}^{+}$, the ratio of $R_{2 n}^{2}$ and $R_{n}^{2}$ is given by

$$
\frac{R_{n}^{2}}{R_{2 n}^{2}} \approx 2^{-2 \nu_{a}}, \quad \text { or } \quad \frac{2^{2 \nu} R_{n}^{2}}{R_{n}^{2}} \approx \begin{cases}1, & \text { if } a<a_{c}^{+} ; \\ 2^{2 \nu-2 \nu_{a}} \approx 2^{-0.32}, & \text { if } a>a_{c}^{+} .\end{cases}
$$

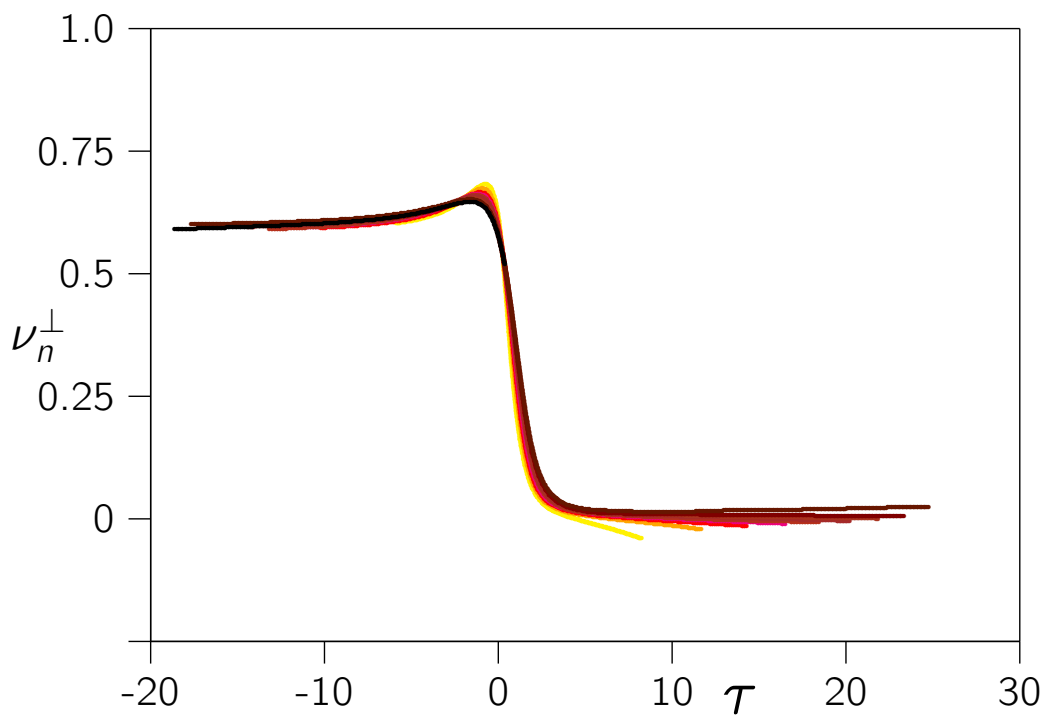

Figure 32: The vertical metric exponent $\nu^{\perp}$ as a function of a for adsorbing walks in $\mathbb{L}_{3}^{+}$. The exponent was estimated from equation (88) by fixing $n$ and then averaging over $m$ for $100 \leq m \leq 500$. With increasing $n$ the exponent changes from $\nu^{\perp}=\nu \approx 0.59$ in the desorbed phase, to $\nu^{\perp}=0$ in the adsorbed phase. The lengths of the walks varied from $n=50$ (yellow) with colours increasing in hue to black when $n=500$ in steps of 50 . 

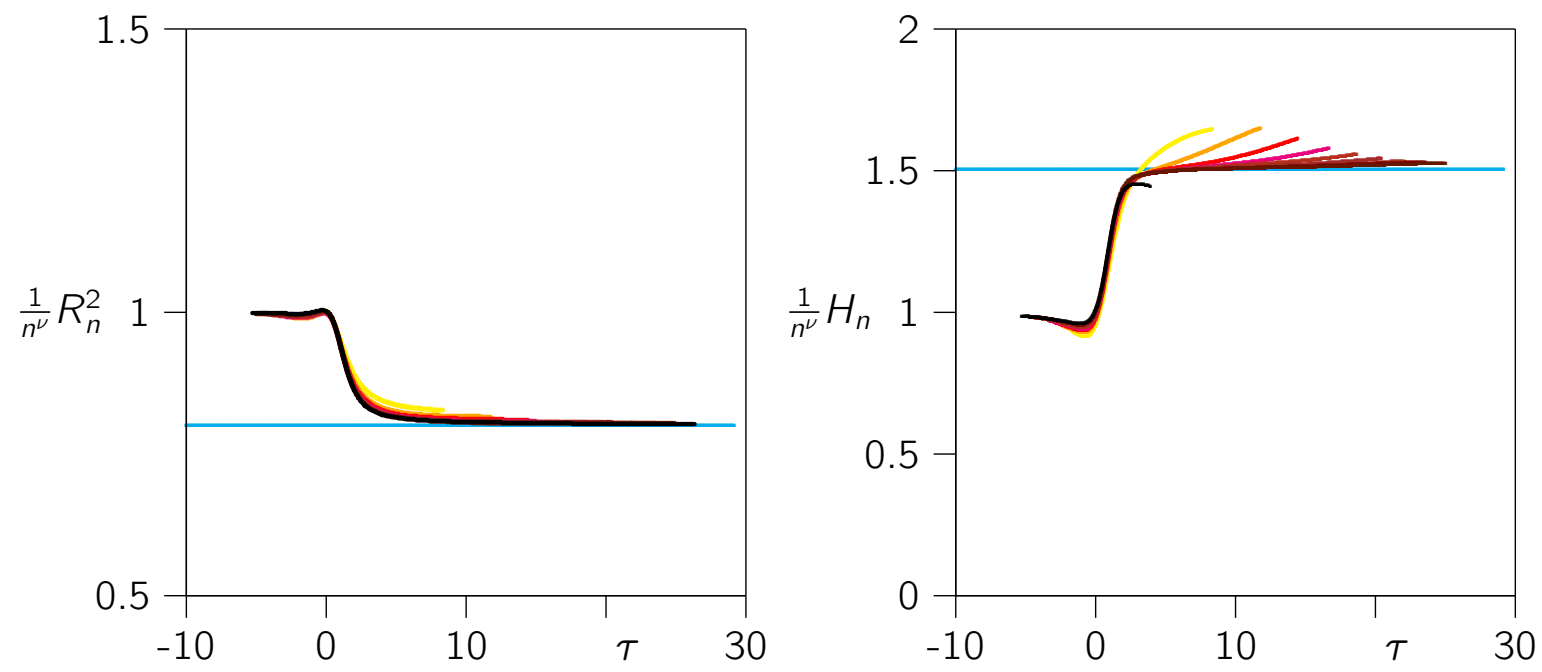

Figure 33: The rescaled mean square radius of gyration $R_{n}^{2} / n^{2 \nu_{a}}$ for adsorbing walks in $\mathbb{L}_{3}^{+}$plotted as a function of $\tau=n^{\phi}\left(a-a_{c}^{+}\right)$is displayed on the left. The metric exponent $\nu_{a}$ is the step-function defined by $\nu_{a}=\frac{3}{4}$ if $a \leq a_{c}^{+}$and $\nu_{a}=1$ if $a>a_{c}^{+}$. The rescaled curves collapse to a single underlying universal scaling function. On the right the similar approach is used to uncover the scaling function for the mean height of the endpoint of the walk, scaled by $n^{\nu_{a}}$. The data are curves for $n \in\{50,100,150, \ldots, 500\}$ increasing in hue from yellow $(n=50)$ to black $(n=500)$.

In figure 33 this is plotted as a function of the rescaled variable $\tau=n^{1 / 2}\left(a-a_{c}^{+}\right)$for $n$ from 25 to 250 in steps of 25 . The curves coincide well with increasing $n$ and signals a transition when $a=a_{c}^{+}$from the desorbed scaling regime into the adsorbed scaling regime. When $a>a_{c}^{+}, 2^{-0.32}=0.801 \ldots$, as shown in the graph. A similar approach using the heights of the endpoint would involve plotting

$$
\frac{H_{n}}{H_{2 n}} \approx 2^{-\nu_{a}}, \quad \text { or } \frac{2^{\nu} H_{n}}{H_{2 n}} \approx \begin{cases}1, & \text { if } a<a_{c}^{+} ; \\ 2^{\nu-\nu^{\perp}} \approx 2^{0.588}, & \text { if } a>a_{c}^{+},\end{cases}
$$

where $\nu^{\perp}$ is the vertical metric exponent. When $a>a_{c}^{+}, 2^{0.588}=1.503 \ldots$, as shown in figure 33 .

3.3.5. The generating function: The generating function of adsorbing walks in the cubic lattice is given by equation (66), where $c_{n}^{+}(v)$ is again the number of walks from the origin of length $n$ in $\mathbb{L}_{+}^{3}$, and with $v$ visits to the adsorbing boundary $\partial \mathbb{L}_{+}^{3}$. Approximations to $G(a, t)$ are given by $G_{N}(a, t)$ in equation (67), and $G_{500}(a, t)$ was calculated using the approximate values of $c_{n}^{+}(v)$ obtained by sampling with the GAS algorithm. The critical curve is given by equation (68) (see figure 18). $G(a, t)$ is singular when $t=t_{c}^{+}(a)$, and if $t>t_{c}^{+}(a)$, then $G(a, t)$ is divergent. In figure 34 the approximation $G_{500}(a, t)$ is plotted, with the location of the critical curve, and critical point $\left(a_{c}^{+}, t_{c}^{+}\right)$indicated (where, as before, $t_{c}^{+}=t_{c}^{+}\left(a_{c}^{+}\right)$). The critical curve is similar to the critical curve in figure 18 , and the critical point divides the critical curve into two curves, namely a curve where the transition is a high temperature or desorbed walk marked by $\tau_{0}$, and a curve where the transition is to a low temperature or adsorbed walk marked by $\lambda$. Along $\tau_{0}$ the critical curve is given by $t_{c}^{+}(a)=\frac{1}{\mu_{3}}$, for $a \leq a_{c}^{+}$and where $\mu_{3}$ is the growth constant of self-avoiding walks in the cubic lattice. 


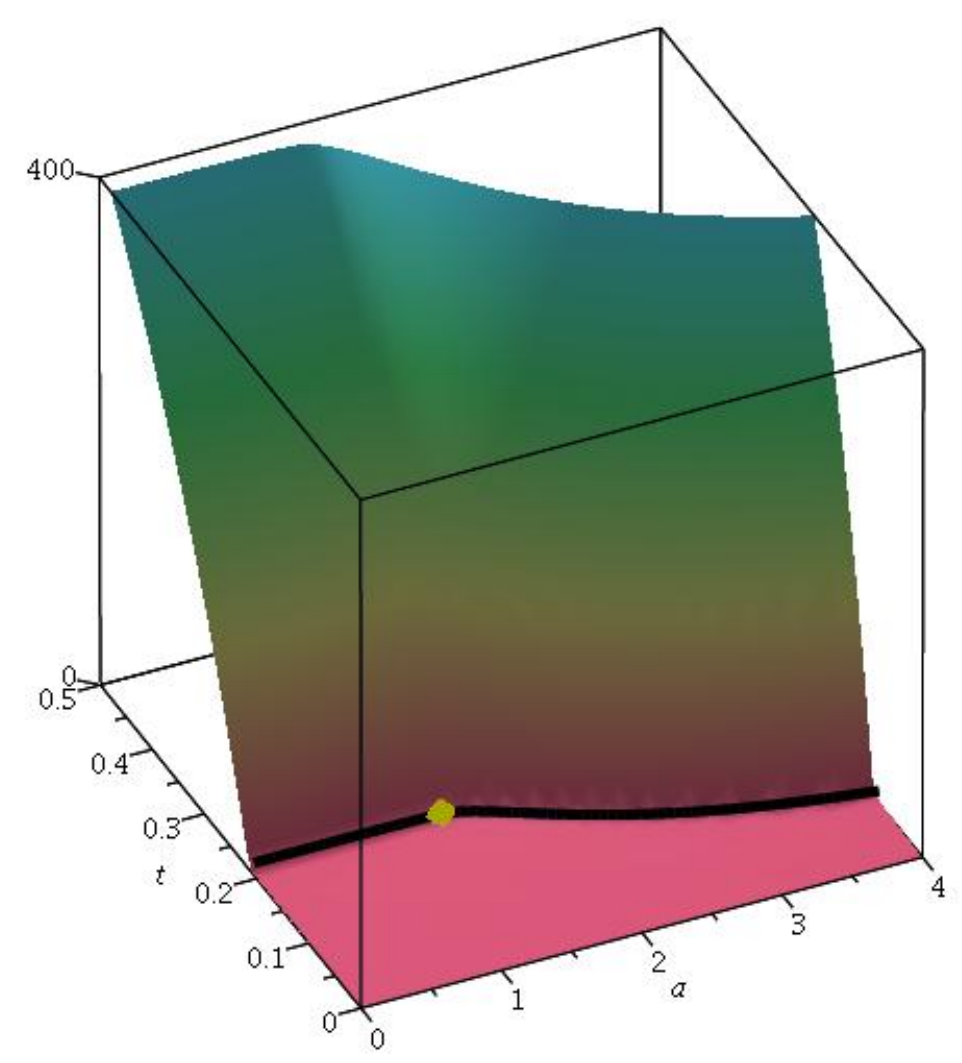

Figure 34: $G_{500}(a, t)$ as a function of $(a, t)$ for adsorbing walks in $\mathbb{L}_{3}^{+}$. The critical curve $t_{c}(a)$ is the black curve, and the location of the critical point at $a=a_{c}^{+}$is indicated. Below the critical curve $G(a, t)$ is finite, and approximated well by $G_{N}(a, t)$ (for large $N$ and not too close to the critical curve). Above the critical curve $G(a, t)$ is divergent, while $G_{N}(a, t)$ is finite.

The critical curve is parametrized by the scaling fields $(\sigma, g)$ as shown in figure 18 . Here, the scaling fields are given by $g=t_{c}^{+}-t$, and $\sigma=a-a_{c}^{+}$. The singular points in $G(a, t)$ along $t_{c}^{+}(a)$ are described by the scaling assumptions shown in equation (69). The exponent $\gamma_{1}$ can be estimated by putting $a=1$ so that $G(1, t) \sim g^{-\gamma_{1}}$. By using the model in equation (70), the estimate

$$
\gamma_{1}=0.725 \ldots
$$

is obtained. This result is close to the estimate 0.697(2) in reference [19]. A similar analysis with $a=a_{c}^{+}$gives the estimate

$$
\gamma_{s}=1.203 \ldots
$$

for the surface exponent at the critical adsorption point. This is slightly smaller than the estimate 1.304(16) in reference [31].

The situation is less clear in the adsorbed phase. The adsorbed walk should have the scaling of a self-avoiding walk in two dimensions, so that $\gamma_{+}$is given by the entropic exponent of walks in $d=2\left(\gamma_{+}=\frac{43}{32}\right)$. Putting $a=4$ and plotting $Z_{n}^{1 / n}(4)$ against $n$ gives an estimate for the critical value $t_{c}^{+}(4)$. In this case the data quickly converges to $t_{c}^{+}(4)=0.0926 \ldots$ (to four decimal places). Assuming that $t_{c}^{+}(4)=0.0926$ and choosing the scaling field $g=(0.0926-t)$ gives a model similar to equation (73). 


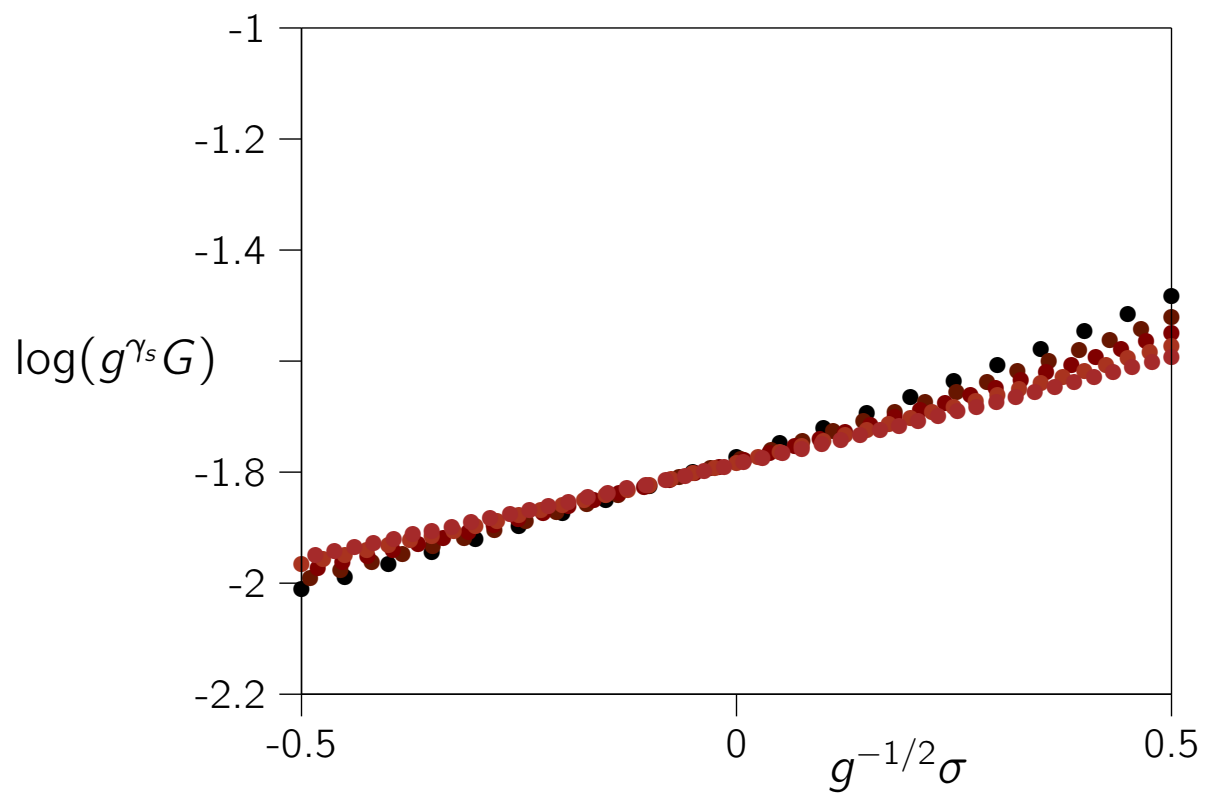

Figure 35: Scaling of the generating function near the critical adsorption point for adsorbing walks in $\mathbb{L}_{3}^{+}$. The data points are above were collected for $g \in[0.01,0.05]$ and $\frac{1}{2} \sqrt{g} \leq \sigma \leq \frac{1}{2} \sqrt{g}$. The value of the exponent $\gamma_{s}$ was assumed to be given by the estimate in equation $(76)$.

Plotting the left hand side as a function of $\frac{1}{\log g}$, and fitting it to a quadratic for $t \in[0,0.06]$, gives the estimate $\gamma_{+} \approx 1.2 \ldots$, still well below the expected result $\frac{43}{32}=1.34375$. Examination of the data shows strong dependence of this result on the range of $g$ in the model. For example, a fit with $t \in[0,0.09]$ gives a smaller value $\gamma_{+} \approx 1.1 \ldots$ These variable results indicate that $G_{500}(a, t)$ is not a good approximation to $G(a, t)$ near the critical curve $\lambda$ in figure 18 for adsorbing walks in the cubic lattice.

In the vicinity of the critical point $\left(a_{c}^{+}, t_{c}^{+}\right)$the generating function should exhibit scaling given by equation (74). Plotting $g^{\gamma_{s}} G(a, t)$ (with $\gamma_{s}=1.203$ ) against the combined variable $g^{-1 / 2} \sigma$ should expose the scaling function $f$. In figure 35 this is done by plotting $\log \left(g^{\gamma_{s}} G(a, t)\right)$ against $g^{-1 / 2} \sigma$ for $g=\frac{1}{\mu_{3}}-t \in[0.01,0.05]$ and $\sigma=\left(a-a_{c}^{+}\right) \in\left[-\frac{1}{2} g^{1 / 2}, \frac{1}{2} g^{1 / 2}\right]$.

The partition function (see equation (6)) also exhibit scaling for large $n$. The scaling assumption in equation (75) applies here as well, where $\log \mu_{a}=\mathcal{F}(a)$ and $\log \mu_{a}=-\log t_{c}(a)$. As before, the exponent $\gamma_{t}$ is related to the $\gamma_{s}$-exponent as in equation (76):

$$
\gamma_{t}-1=\gamma_{s}-1 \approx 1.203-1=0.203 .
$$

That is, when $a$ is close to $a_{c}^{+}$(and $a<a_{c}^{+}$), then the partition function has asymptotic behaviour

$$
Z_{n}(a) \simeq n^{0.203} h\left(n^{\phi}\left(a_{c}^{+}-a\right)\right) \mu_{a}^{n} .
$$

This result may be tested by plotting $n^{0.203} Z_{n}\left(a_{c}^{+}\right)\left(t_{c}(a)\right)^{n}$ against $\tau=n^{\phi}\left(a-a_{c}^{+}\right)$. This scaling is seen in figure 36 for $a<a_{c}^{+}$and $n \in\{50,100,150, \ldots, 500\}$; all the data accumulate along a single curve, exposing the scaling function $h$. 


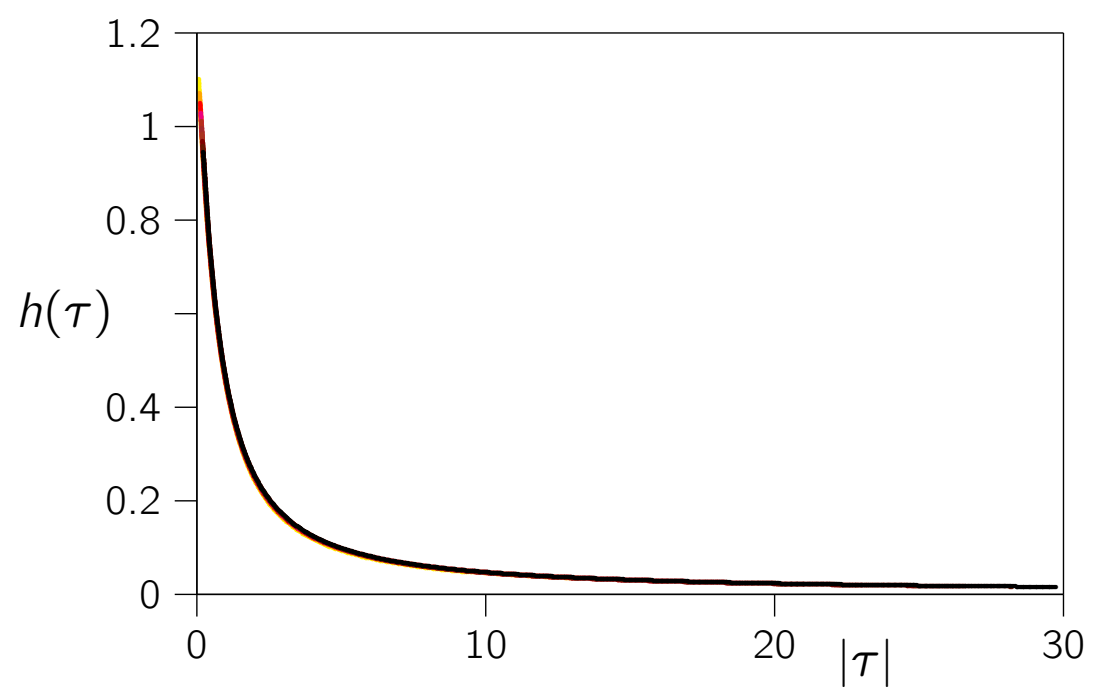

Figure 36: The scaling of the partition function as suggested by equation (94) for adsorbing walks in $\mathbb{L}_{3}^{+}$. The curves are plots of $n^{-0.203} Z_{n}(a) \mu^{-n}$ as a function of $|\tau|=n^{1 / 2}\left|a-a_{c}^{+}\right|$(with $a_{c}^{+}$approximated by 1.33 and $a<a_{c}^{+}$). All the data collapse to a single curve, which is the scaling function $h$ in equation (94). The lengths of the walks varied from $n=50$ (yellow) with colours increasing in hue to black when $n=500$ in steps of 50 .

\section{Conclusions}

The adsorbing self-avoiding walk is a classical model in rigorous and numerical statistical mechanics, and have received considerable attention in the physics and mathematics literature [12, 18, 19, 23, 29].

In this paper the feasibility of collecting data in the microcanonical ensemble on adsorbing walks using a flat histogram implementation of the GAS algorithm [24] was considered. This is an approximate enumeration algorithm, and the data can be used to directly estimate partition and generating functions, from which thermodynamic functions such as the free energy and specific heat can be determined (see, for example, reference [22]).

The implemementation of the algorithm was done using endpoint elementary moves on half space self-avoiding walks, and the algorithm sampled from a flat histogram with reasonable success in both length and energy in the square and cubic lattices. Analysis of the data gives good results, better than previous Monte Carlo simulations in, for example, references [19, 23], but not as good as exact enumeration data in references [3, 4]. A significant advance of this algorithm is that its produces a large amount of microcanonical data. Modifications to obtained data with respect to other quantities are trivial, and can easily be implemented. The simulations reported here were done on a Dell Inspiron 530 desktop machine, but note that the algorithm can be implemented in parallel on a cluster with each cluster generating an independent sequence. This should give radically improved statistical data.

The success of the implementation suggests that this numerical method may be used on other models (collapsing self-avoiding walks [30, 39], for example). However, it may be necessary to extend the method by introducing, in addition to the sets of parameters 
denoted by $\left\{\beta_{\ell, u}\right\}$ and $\left\{\gamma_{\ell, u}\right\}$, additional sets of parameters which are conjugate to classes of elementary moves. For example, in the model of collapsing walks (see figure 4), the energy may be changed by $\Delta u \in\{-2 d+1,-2 d+2, \ldots, 2 d-1\}$ by an elementary move, and parameters may be introduced for each value of $\Delta u$ to achieve flat histogram sampling (in a way similar to the introduction of $\gamma_{\ell, u}$ for elementary moves increasing the energy of the walk. This will increase the complexity of the implementation, but with the result that flat histogram sampling will be easier to achieve.

In the models of square and cubic lattice adsorbing walks, the algorithm produced data which gave good estimates of the locations of the critical adsorption point. The best estimates are obtained from equations (53) and (79), namely

$$
a_{c}^{+}= \begin{cases}1.779 \pm 0.003, & \text { in the square lattice } \\ 1.306 \pm 0.007, & \text { in the cubic lattice. }\end{cases}
$$

These results can be used to estimate the crossover exponent $\phi$ association with the adsorption transition, and our best esimates are seen in equations (54) and (80):

$$
\phi= \begin{cases}0.496 \pm 0.009, & \text { in two dimensions; } \\ 0.505 \pm 0.006, & \text { in three dimensions. }\end{cases}
$$

In addition, other quantities from which $a_{c}^{+}$and $\phi$ can be estimated were examined, and results largely consistent with the above values were obtained (see, for example, equations (55), (58) and (60) for square lattice results, and equations (81), (83) and (85) for cubic lattice results). These numerical estimates are in good agreement with those presented in reference [23], and also in reference [3] in the case of the square lattice. The estimate in this reference, obtained from exact series data, namely $a_{c}^{+}=1.77564$, agrees with the estimate above to two decimal places. In the cubic lattice the estimate for $a_{c}^{+}$above is slightly smaller than the estimates $a_{c}^{+}=1.338 \pm 0.005$ [31] and $a_{c}^{+}=1.334 \pm 0.027$ in reference [23] (rounding up of this last error bar gives a confidence interval which includes 1.306).

The signature of the adsorption transition in the metric quantities of the model was also examined. The scaling of these quantities with $\tau=n^{\phi}\left(a-a_{c}^{+}\right)$were plotted in figures 14. 16 and 17 in the square lattice, and in figures 30,32 and 33 in the cubic lattice. These results show a transition strongly characterised by changes in metric scaling and verify the value of the metric exponent and its finite size scaling through the critical point.

Finally, the scaling of the generating function and partition function in these models were examined. Our results strongly supports the conventional properties of the model, and the values of the exponents $\left\{\gamma_{1}, \gamma_{s}, \gamma_{+}\right\}$estimated here are consistent with exact values and other estimates in the literature.

The results in the square lattice are consistent with the exact values of $\gamma_{1}$ and the surface exponent $\gamma_{s}$, and the generating and partition partition function exhibit scaling consistent with the value of $\gamma_{s}$, as shown in figures 20 and 21 . In the cubic lattice our data gave the estimates $\gamma_{1} \approx 0.725$ and $\gamma_{s} \approx 1.203$. These values are in addition to estimates elsewhere in the literature (see references [19], [31]), and although the estimates here may be improvements on previous esimates, they remain uncertain. However, scaling of 
the generating function in figure (35), and of the partition function in figure 36, is some evidence that the esimate for $\gamma_{s}$ is at least consistent with the scaling in the model.

Acknowledgements: EJJvR acknowledges financial support from NSERC (Canada) in the form of a Discovery Grant.

\section{References}

[1] C Aragao de Carvalho and S Caracciolo. A new Monte Carlo approach to the critical properties of self-avoiding random walks. J de Phys, 44:323-331, 1983.

[2] MT Batchelor and CM Yung. Exact results for the adsorption of a flexible self-avoiding polymer chain in two dimensions. Phys Rev Lett, 74:2026-2029, 1995.

[3] NR Beaton, AJ Guttmann, and I Jensen. Two-dimensional self-avoiding walks and polymer adsorption: critical fugacity estimates. J Phys A: Math Theo, 45:055208, 2012.

[4] D Bennett-Wood and AL Owczarek. Exact enumeration results for self-avoiding walks on the honeycomb lattice attached to a surface. J Phys A: Math Gen, 29:4755-4768, 1996.

[5] B Berg and D Foerster. Random paths and random surfaces on a digital computer. Phys Lett $B$, 106:323-326, 1981.

[6] A Berretti and AD Sokal. New Monte Carlo method for the self-avoiding walk. J Stat Phys, 40:483531, 1985.

[7] HD Bijsterbosch, VO De Haan, AW De Graaf, M Mellema, FAM Leermakers, MA Cohen Stuart, and AA van Well. Tethered adsorbing chains: Neutron reflectivity and surface pressure of spread diblock copolymer monolayers. Langmuir, 11(11):4467-4473, 1995.

[8] K Binder, C Domb, and MS Green. Critical behaviour at surfaces. In C Domb and JL Lebowitz, editor, Phase Transitions and Critical Phenomena, volume 8, pages 1-144. Academic Press, 1983.

[9] TW Burkhardt, E Eisenriegler, and I Guim. Conformal theory of energy correlations in the semiinfinite two-dimensional $O(n)$ model. Nucl Phys B, 316:559-572, 1989.

[10] JL Cardy. Conformal invariance. In C Domb and JL Lebowitz, editor, Phase Transitions and Critical Phenomena, volume 11, pages 55-126. Academic Press, 1983.

[11] N Clisby. Accurate estimate of the critical exponent $\nu$ for self-avoiding walks via a fast implementation of the pivot algorithm. Phys Rev Lett, 104:055702, 2010.

[12] P-G de Gennes. Scaling Concepts in Polymer Physics. Cornell, 1979.

[13] EA DiMarzio and RJ Rubin. Adsorption of a chain polymer between two plates. J Chem Phys, 55:4318-4336, 1971.

[14] B Duplantier. Statistical mechanics of polymer networks of any topology. J Stat Phys, 54:581-680, 1989.

[15] P Grassberger. Pruned-enriched Rosenbluth method: Simulations of $\theta$ polymers of chain length up to 1000000. Phys Rev E, 56:3682-3693, 1997.

[16] JM Hammersley. Percolation processes II. The connective constant. Proc Camb Phil Soc, 53:642645, 1957.

[17] JM Hammersley. Limiting properties of numbers of self-avoiding walks. Phys Rev, 118:656-656, 1960.

[18] JM Hammersley, GM Torrie, and SG Whittington. Self-avoiding walks interacting with a surface. J Phys A: Math Gen, 15:539-571, 1982.

[19] R Hegger and P Grassberger. Chain polymers near an adsorbing surface. J Phys A: Math Gen, 27:4069-4081, 1994.

[20] H-P Hsu and P Grassberger. A review of Monte Carlo simulations of polymers with perm. J Stat Phys, 144:597-637, 2011.

[21] EJ Janse van Rensburg. Collapsing and adsorbing polygons. J Phys A: Math Gen, 31(41):8295-8306, 1998.

[22] EJ Janse van Rensburg. The statistical mechanics of interacting walks, polygons, animals and vesicles. 
Oxford Univesity Press, 2 edition, 2015.

[23] EJ Janse van Rensburg and A Rechnitzer. Multiple markov chain Monte Carlo study of adsorbing self-avoiding walks in two and in three dimensions. J Phys A: Math Gen, 37:6875-6898, 2004.

[24] EJ Janse van Rensburg and A Rechnitzer. Generalized atmospheric sampling of self-avoiding walks. J Phys A: Math Theo, 42:335001, 2009.

[25] EJ Janse van Rensburg and A Rechnitzer. Generalized atmospheric sampling of knotted polygons. J Knot Theo Ram, 20(08):1145-1171, 2011.

[26] EJ Janse van Rensburg and A Rechnitzer. On the universality of knot probability ratios. J Phys A: Math Theo, 44(16):162002, 2011.

[27] LI Klushin, AA Polotsky, H-P Hsu, DA Markelov, K Binder, and AM Skvortsov. Adsorption of a single polymer chain on a surface: Effects of the potential range. Phys Rev E, 87:022604, 2013.

[28] J Krawczyk, T Prellberg, AL Owczarek, and A Rechnitzer. Stretching of a chain polymer adsorbed at a surface. Journal of Statistical Mechanics: Theory and Experiment, 2004(10):P10004, 2004.

[29] S Livne and H Meirovitch. Computer simulation of long polymers adsorbed on a surface I. Corrections to scaling in an ideal chain. J Chem Phys, 88:4498-4506, 1988.

[30] H Meirovitch and HA Lim. The collapse transition of self-avoiding walks on a square lattice: a computer simulation study. J Chem Phys, 91:2544-2552, 1989.

[31] H Meirovitch and S Livne. Computer simulation of long polymers adsorbed on a surface II. Critical behavior of a single self-avoiding walk. J Chem Phys, 88:4507-4515, 1988.

[32] S Mishra, A Bera, and Mandal A. Effect of polymer adsorption on permeability reduction in enhanced oil recovery. J Petrol Eng, 2014:395857, 2014.

[33] T Prellberg and J Krawczyk. Flat histogram version of the pruned and enriched Rosenbluth method. Phys Rev Lett, 92:120602, 2004.

[34] T Prellberg and AL Owczarek. Four-dimensional polymer collapse: pseudo-first-order transition in interacting self-avoiding walks. Physical Review E, 62(3):3780-3789, 2000.

[35] V Privman, G Forgacs, and HL Frisch. New solvable model of polymer-chain adsorption at a surface. Phys Rev B, 37:9897-9900, 1988.

[36] A Rechnitzer and EJ Janse van Rensburg. Generalized atmospheric Rosenbluth methods (GARM). J Phys A: Math Theo, 41:442002, 2008.

[37] MN Rosenbluth and AW Rosenbluth. Monte Carlo calculation of the average extension of molecular chains. J Chem Phys, 23:356-359, 1955.

[38] G Rychlewski and SG Whittington. Self-avoiding walks and polymer adsorption: low temperature behaviour. Journal of Statistical Physics, 145(3):661-668, 2011.

[39] MC Tesi, EJ Janse van Rensburg, E Orlandini, and SG Whittington. Monte Carlo study of the interacting self-avoiding walk model in three dimensions. J Stat Phys, 82:155-181, 1996.

[40] SG Whittington. A directed-walk model of copolymer adsorption. J Phys A: Math Gen, 31:87978803, 1998. 